I N T ER N ATIONAL MONETARY FUND

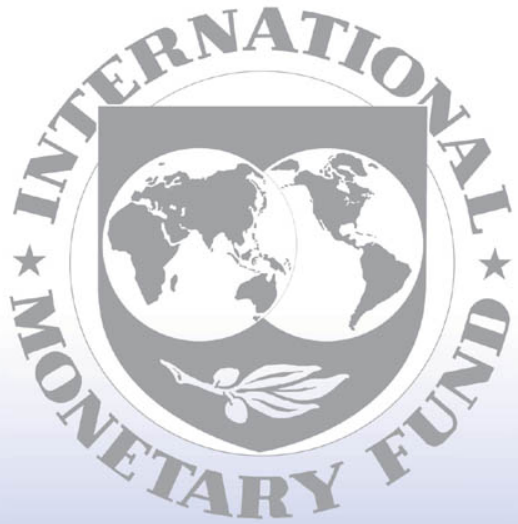

Staff

Country

Reports 


\section{Rwanda: 2006 Article IV Consultation, First Review Under the Three-Year Arrangement Under the Poverty Reduction and Growth Facility, and Request for Waiver of Nonobservance of Performance Criteria-Staff Report; Staff Statement; Public Information Notice and Press Release on the Executive Board Discussion; and Statement by the Executive Director for Rwanda}

Under Article IV of the IMF's Articles of Agreement, the IMF holds bilateral discussions with members, usually every year. In the context of a combined discussion of the 2006 Article IV consultation with Rwanda, the first review under the three-year arrangement Under the Poverty Reduction and Growth Facility, and request for a waiver of nonobservance of performance criteria, the following documents have been released and are included in this package:

- the staff report for the combined 2006 Article IV Consultation, First Review Under the Three-Year Arrangement Under the Poverty Reduction and Growth Facility, and Request for Waiver of Nonobservance of Performance Criteria, prepared by a staff team of the IMF, following discussions that ended on November 3, 2006, with the officials of Rwanda on economic developments and policies. Based on information available at the time of these discussions, the staff report was completed on January 10, 2007. The views expressed in the staff report are those of the staff team and do not necessarily reflect the views of the Executive Board of the IMF;

- $\quad$ a staff statement of January 29, 2007 updating information on recent economic developments;

- a Public Information Notice (PIN) and Press Release, summarizing the views of the Executive Board as expressed during its January 29, 2007, discussion of the staff report on issues related to the Article IV consultation and the IMF arrangement, respectively; and

- $\quad$ a statement by the Executive Director for Rwanda.

The documents listed below have been or will be separately released.

Letter of Intent sent to the IMF by the authorities of Rwanda*

Memorandum of Economic and Financial Policies by the authorities of Rwanda*

Technical Memorandum of Understanding*

*Also included in Staff Report

The policy of publication of staff reports and other documents allows for the deletion of market-sensitive information.

To assist the IMF in evaluating the publication policy, reader comments are invited and may be sent by e-mail to publicationpolicy@imf.org.

Copies of this report are available to the public from

International Monetary Fund $\bullet$ Publication Services

$70019^{\text {th }}$ Street, N.W. $\bullet$ Washington, D.C. 20431

Telephone: (202) 623-7430 • Telefax: (202) 623-7201

E-mail: publications@imf.org•Internet: http://www.imf.org

Price: $\$ 18.00$ a copy

International Monetary Fund

Washington, D.C.

CInternational Monetary Fund. Not for Redistribution 
This page intentionally left blank

CInternational Monetary Fund. Not for Redistribution 
INTERNATIONAL MONETARY FUND

\title{
RWANDA
}

\section{Article IV Consultation, First Review Under the Three-Year Arrangement Under the Poverty Reduction and Growth Facility, and Request for Waiver of Nonobservance of Performance Criteria}

\author{
Prepared by the African Department \\ (In collaboration with other departments) \\ Approved by Robert Corker and Mark Plant
}

January 10, 2007

\begin{tabular}{l} 
PRGF/Debt Relief. The Executive Board approved a PRGF arrangement with access \\
of 10 percent of quota on June 5, 2006. Rwanda reached the completion point under \\
the enhanced HIPC Initiative in April 2005 and qualified for the MDRI in January \\
2006. \\
2004 Article IV consultation. At the Article IV Board meeting on October 6, 2004, \\
Directors identified major challenges ahead in sustaining economic growth to reduce \\
poverty while achieving fiscal and external sustainability. They urged the authorities \\
to strengthen the financial sector, improve tax efficiency, and limit external \\
borrowing. \\
Main topics. The report recommends completion of the first review under the PRGF. \\
Article IV discussions centered on obstacles to growth, enhancing competitiveness \\
and, with Rwanda expected to be among the first recipients of any scaled-up aid, \\
macroeconomic challenges from a scaling up and preparations for effective use of \\
aid. Selected Issues Papers were written on the latter two topics. \\
Exchange system. Rwanda adopted a market-determined exchange rate system in \\
March 1995. However, for more than a year, the exchange rate has in fact behaved \\
like a conventional fixed peg. Rwanda has accepted obligations under Article VIII, \\
Sections 2, 3 and 4, and maintains a system free of restrictions on the making of \\
payments and transfers for current international transactions. \\
Participants. The mission met with Minister of Finance and Economic Planning \\
Musoni, Governor Kanimba of the National Bank of Rwanda, other government \\
officials, and representatives of the private sector, civil society, and the international \\
community during October 18-November 3, 2006. The mission team headed by \\
Ms. Kostial consisted of Mr. Darius Mr. Mitchell, and Ms. Kaendera (all AFR) and \\
was assisted by Mr. Engström, the resident representative. \\
\hline
\end{tabular}


Executive Summary

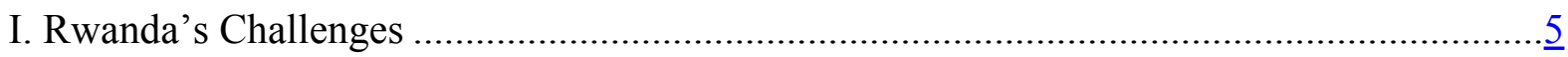

II. The Medium Term —Stimulating Growth.....................................................................

A. Removing Obstacles to Growth ......................................................................

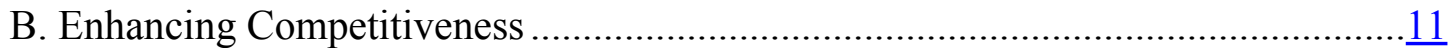

C. Boosting Growth —A Scenario for Scaling-Up ...................................................

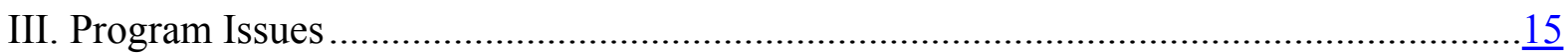

A. On Track, But There Are Pressures …………...............................................15

B. The Medium-Term Framework ....................................................................

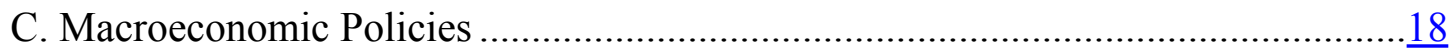

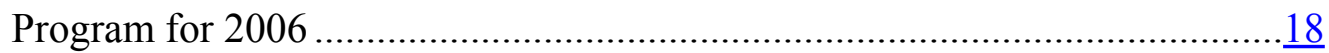

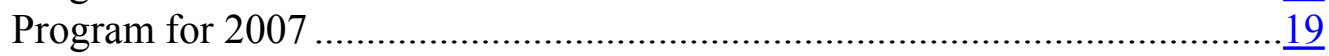

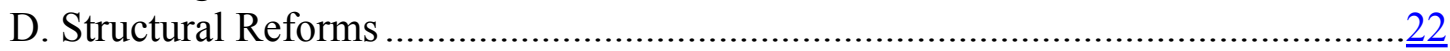

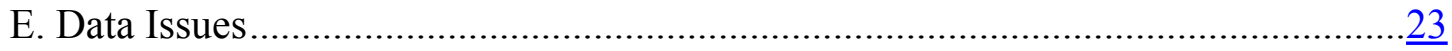

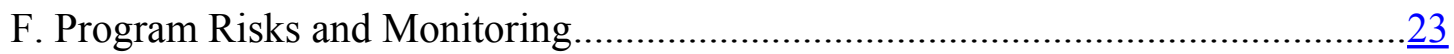

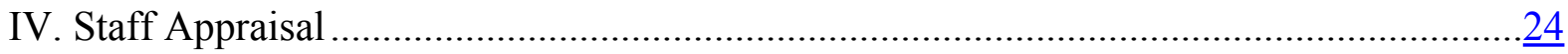

Boxes

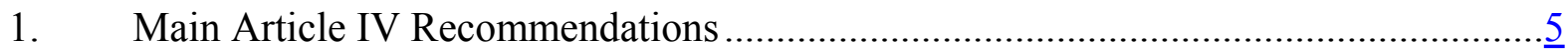

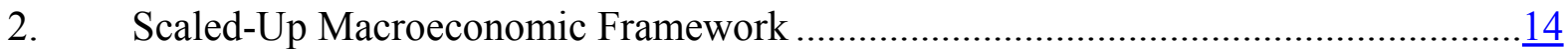

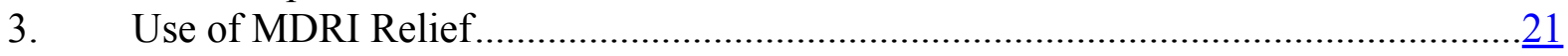

Figure

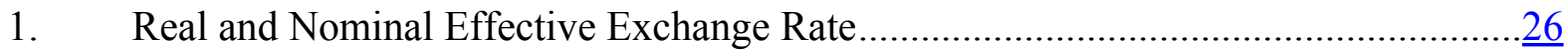

Tables

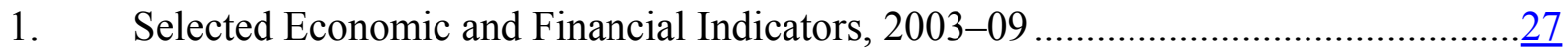

2. Operations of the Central Government, 2005-09 ….............................................

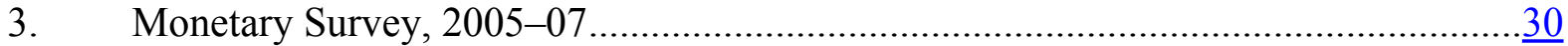

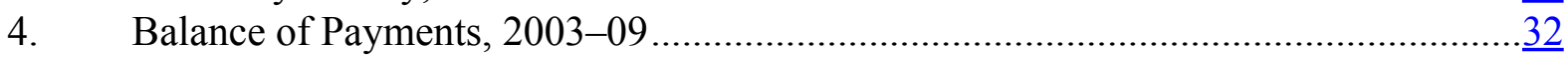


5. External Financing Requirements and Sources, 2003-09 ……................................33

6. External Public Debt and Debt Service, 2003-09 ..................................................

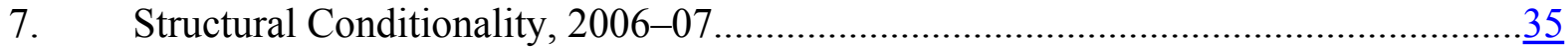

8. Financial Soundness Indicators for the Banking Sector, 2000-05 …….....................

Appendices

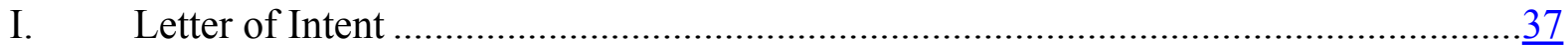

Attachment I. Memorandum of Economic and Financial Policies of of the Government of Rwanda(2007) ............................................................

Attachment II. Technical Memorandum of Understanding …….........................57

II. Millennium Development Goals....................................................................... 


\section{EXECUTIVE SUMMARY}

Rwanda must increase its growth rates in order to reduce poverty. Since the 1994 genocide national rehabilitation has moved forward, output has recovered and, with the help of debt relief, macroeconomic stability has been largely achieved. However, per capita growth has recently slowed and poverty is pervasive. Stimulating growth would require prioritizing policies to address Rwanda's severe infrastructure gap and low agricultural yields, enhance competitiveness, and continue social and structural reforms. The PRSP update planned for 2007 is expected to address these issues. Prospects for scaling-up aid are encouraging.

Policy implementation has been satisfactory. Growth is expected to exceed 4 percent because activity is buoyant in the manufacturing, services, and financial sectors. However, reflecting primarily a rise in food and energy prices, 12-month inflation gradually increased to 11 percent in November. Macroeconomic performance is in line with the program, but short-term risks have evolved on the monetary side from a large increase in private sector credit and the central bank's foreign exchange intervention policy. Structural reforms are broadly on track. All but two performance criteria (PCs) for the first review were met. The quantitative end-June PC on priority spending and the structural end-August PC on improving the monitoring of projects accounts were missed; because both deviations were temporary, staff recommends waivers.

\section{The greatest challenge for Rwanda in 2007 is to absorb a scaling up of aid while preserving macroeconomic stability. With indications of real appreciation already} occurring in 2006, managing the impact of fiscal policies on domestic demand will be all the more important if aid is scaled up, as expected, in 2007. Acknowledging large development needs, the program allows for an increase in the deficit (excluding spending on peacekeeping and demobilization) by about 1 percent of GDP. Grants are likely to be higher than programmed, but the program would accommodate any further expansion in the deficit only after a review has established that the expansion would not lead to a rekindling of inflation or undue crowding out of the private sector. The central bank has committed to closely monitor credit and inflationary developments and tighten its monetary stance if necessary.

The structural agenda is designed to address impediments to growth. Reforms will focus on the financial sector, private sector development, public expenditure management, and establishing a poverty profile for Rwanda.

Program risks have increased. The main risk is that the monetary program could go off track if the central bank resists pressures for an underlying exchange rate appreciation. A new risk has emerged on the security front because Rwanda may have to cope with refugees from neighboring countries. 


\section{RWANDA'S ChaLlengeS}

1. Rwanda has made great strides since the $\mathbf{1 9 9 4}$ genocide. National rehabilitation has moved forward. There were presidential and legislative elections in 2003, and there have been community-based hearings of those accused of genocide. Output has recovered and, with the help of debt relief, macroeconomic stability has been largely achieved. Since the 2004 Article IV consultation the authorities' program, supported by PRGF arrangements, has been broadly on track and Rwanda has made good progress in responding to Fund advice (Box 1).

\begin{tabular}{|l|l|}
\hline \multicolumn{2}{|c|}{ Box 1. Main Article IV Recommendations } \\
\hline \multicolumn{1}{|c|}{ Advice } & \multicolumn{1}{|c|}{ Status } \\
\hline $\begin{array}{l}\text { Strengthen economic legislation to improve } \\
\text { tax efficiency. }\end{array}$ & $\begin{array}{l}\text { Laws on income and tax procedures were passed in } \\
\text { 2005 and implemented in January 2006. In April } 2006 \\
\text { a customs law was adopted. }\end{array}$ \\
\hline Reform the civil service. & $\begin{array}{l}\text { The core civil service has been streamlined. Reforms } \\
\text { are being extended to the entire civil service. }\end{array}$ \\
\hline $\begin{array}{l}\text { Strengthen financial intermediation and } \\
\text { bank soundness. }\end{array}$ & $\begin{array}{l}\text { On the basis of action plans drafted with the National } \\
\text { Bank of Rwanda (NBR), five of six commercial banks } \\
\text { have been brought into compliance with prudential } \\
\text { regulations and agreement has been reached on } \\
\text { restructuring the remaining problem bank. } \\
\text { Amendments to the banking law to strengthen the } \\
\text { supervisory powers of the central bank were submitted } \\
\text { to parliament in September 2006. }\end{array}$ \\
\hline Reform land tenure. & $\begin{array}{l}\text { Parliament in July 2005 approved a land law conferring } \\
\text { security of tenure and user rights; subordinate } \\
\text { legislation and regulations are being drafted. The law } \\
\text { will be implemented through a consultative process } \\
\text { over several years. }\end{array}$ \\
\hline Privatize public commercial enterprises. & $\begin{array}{l}\text { The telecommunications carrier, Rwandatel, was } \\
\text { privatized in June 2005; majority shares of two banks } \\
\text { were sold to foreign investors in December 2004; and } \\
\text { the largest coffee exporter and three tea factories } \\
\text { (among others) were privatized. }\end{array}$ \\
\hline
\end{tabular}


On the Road to Macroeconomic Stability After the Genocide
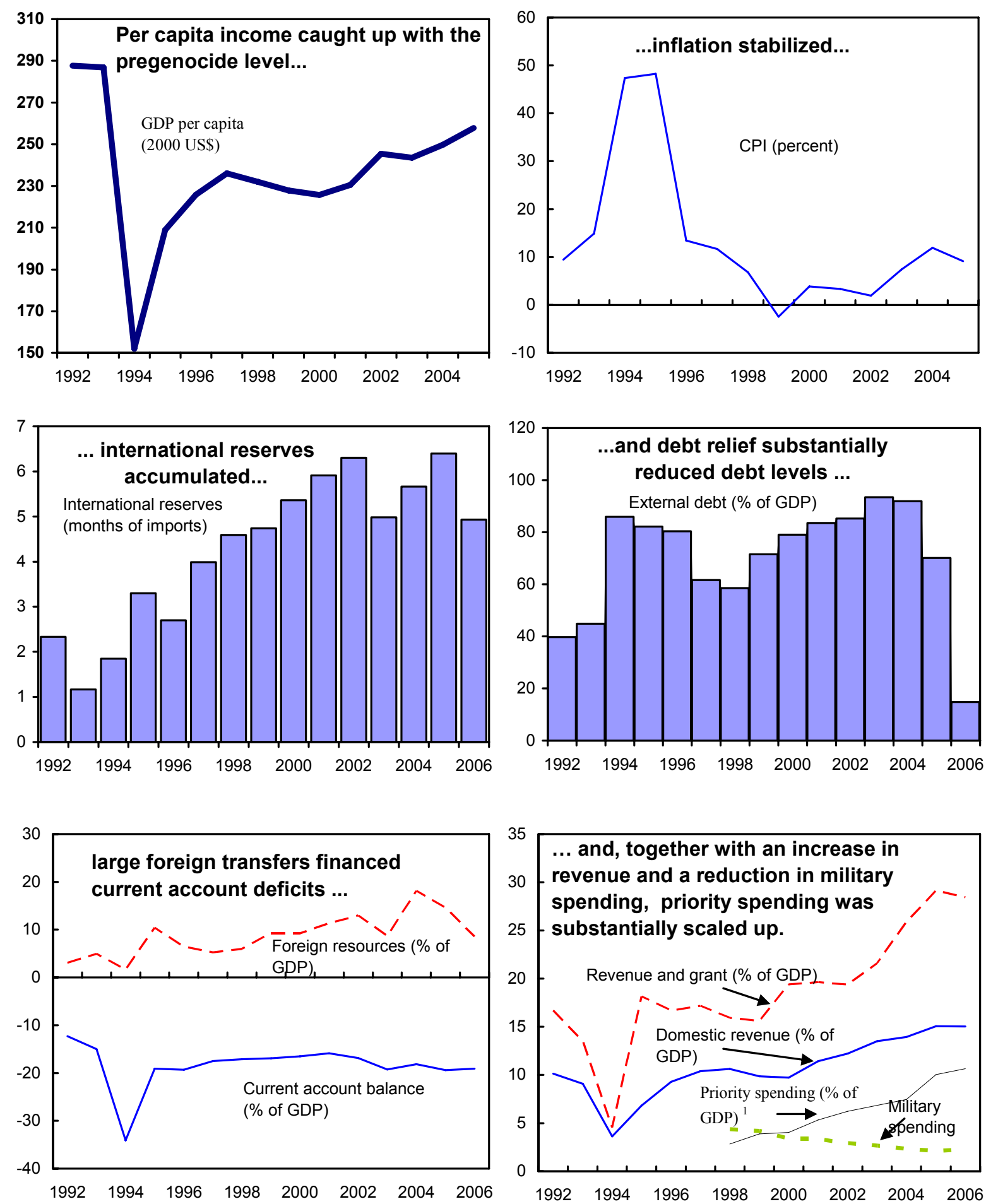

Source: The WDI database, Rwandese authorities and IMF staff.

${ }^{1}$ In 2005 , the definition of priority spending was amended to include the electricity sector. 
2. Nonetheless, poverty is still pervasive. The living standards of the poor are well below those in other members of the East African Community (EAC). While Rwanda has already met three MDG goals (immunization, reduction of HIV/AIDS prevalence, and elimination of the gender gap in primary education), the World Bank believes that if it is to achieve the rest, it will require substantial additional funding. It is unlikely that some goals can be met, particularly that of halving the proportion of people living on less than US\$1 a day (Appendix II).
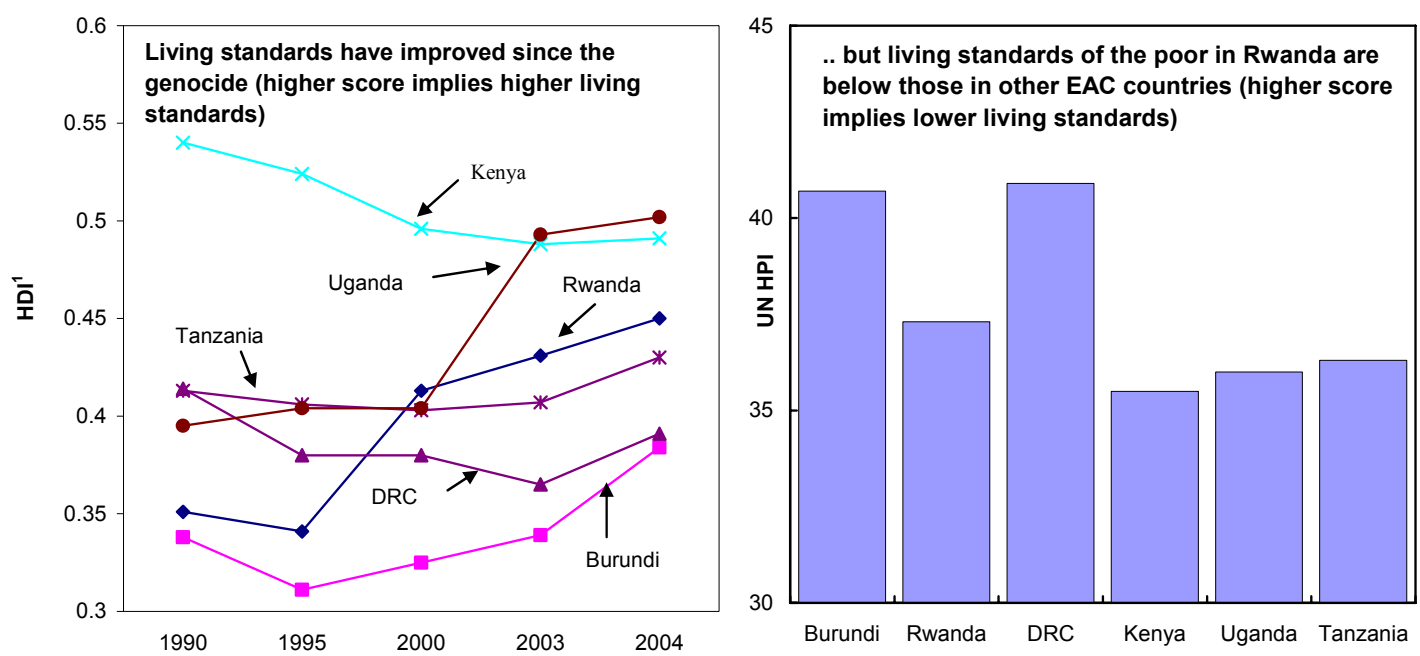

Source: UN Human Development Report, 2006.

\footnotetext{
${ }^{1}$ The UN HDI ( Human Development Index) measures achievemerits in human development by taking into account life expectancy at birth, the adult literacy rate, the combined primary, secondary and tertiary gross school enrolment ratio, and the GDP per capita. The higher the score, the better the situation in a country.

${ }^{2}$ The UN HPI (Human Poverty Index) is designed for developing cciuntries. The HPI measures deprivations in human development by taking into account the probability at birth of not surviving to age 40 , the adult literacy rate, lack of access to water, and the percentage of underweight children. The higher the score, the worse the situation in a country.
}

3. The question now is how to increase real growth so as to reduce poverty. The catch-up effect following the genocide has been waning in recent years and per capita growth has fallen to only 2 percent per year. Growth at this rate cannot bring the next generation out of poverty.

\section{The Medium Term-STIMUlating GrowTH}

4. The Article IV consultation concentrated on how to boost growth. To make headway in halving poverty by 2015 notwithstanding high population growth, real GDP growth must be about doubled. This will require raising total factor productivity growth by investing in human and physical capital and further advancing structural reforms.

Discussions thus centered on obstacles to growth, enhancing competitiveness, and, with 
Rwanda expected to be among the first recipients of scaled-up aid, the macroeconomic challenges that scaling up would bring.

\section{A. Removing Obstacles to Growth}

5. General consensus on the main obstacles to growth exists. ${ }^{1}$ After consultations earlier in 2006 in the context of an evaluation of the PRSP implementation, stakeholders now generally agree on obstacles to growth. Macroeconomic stability and debt sustainability are recognized as prerequisites for strong growth, but the discussions also reflected increasing recognition that, until Rwanda's severe infrastructure gap is addressed, progress toward its medium-term objectives will be limited. This recognition is expected to become the main theme of the PRSP update planned for 2007. There is also consensus that security concerns in the Great Lakes region are undermining investor confidence.

Private sector activity in Rwanda is hampered by weak infrastructure, high cost of doing business, and human resource constraints.

\section{Physical Infrastructure}

Transport cost (US\$/ton)

Electricity consumption per capita (Kwh)

Fixed and mobile telephone subscribers (per 1,000 persons)

Electricity cost (business use, US\$/Kwh)

\section{Work Force}

Health expenditure (per capita)

Adult literacy (\%) Labor market rigidity index ( $100=$ highest rigidity $)$

\begin{tabular}{|c|c|c|c|c|}
\hline Rwanda & Uganda & Tanzania & LDCs & Kenya \\
\hline 165.0 & 95.0 & $\ldots$ & $\ldots$ & $\ldots$ \\
\hline 14.0 & 51.7 & 54.4 & 112.4 & 124.9 \\
\hline 17.0 & 32.0 & 31.0 & 23.0 & 58.0 \\
\hline 0.20 & 0.08 & 0.06 & $\ldots$ & 0.08 \\
\hline 7.0 & 18.0 & 12.0 & $\ldots$ & 20.0 \\
\hline 64.0 & 68.9 & 69.4 & $\ldots$ & 73.6 \\
\hline 59.0 & 13.0 & 69.0 & $\ldots$ & 28.0 \\
\hline
\end{tabular}

Source: World Development Indicators, United Nations, and World Fact Book.

Note: The data in the table reflect the latest information available between 2002-2004.

\footnotetext{
${ }^{1}$ Governance was not considered an impediment even though the recent World Governance Indicators show a substantial deterioration in Rwanda's score for 2005. Donors and private sector representatives expressed reservations about the quality of the index. The 2006 report of the African Peer Review Mechanism noted significant progress.
} 


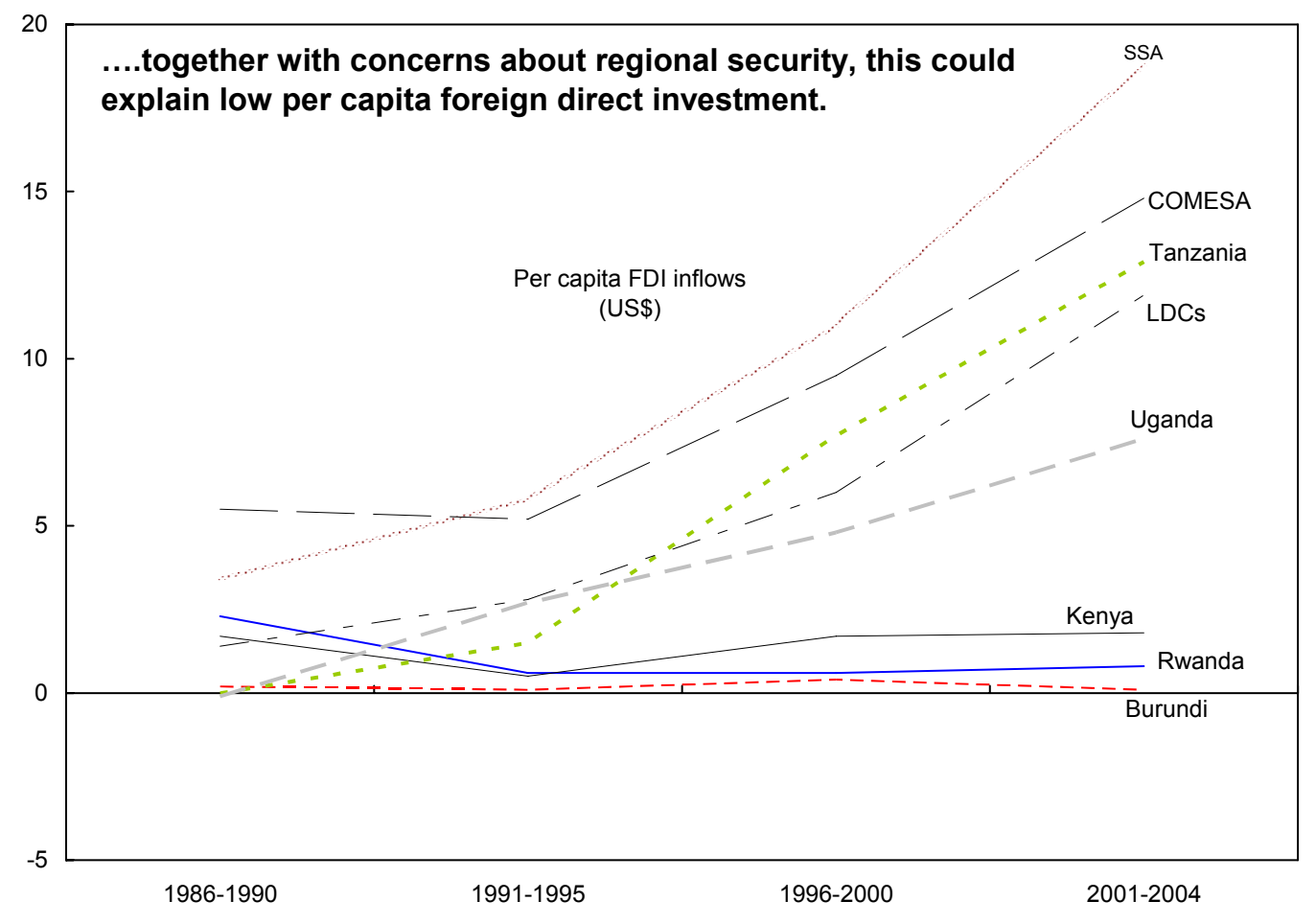

\section{Agricultural yields are}

low. Improving food security is critical, since Rwanda is vulnerable to shocks, and has little scope to increase arable land. Experience in a Millennium Village $^{2}$ points to a tripling of yields with proper fertilizer and seeds. However, the agricultural strategy is not sufficiently advanced to be put into operation on a large scale, primarily because

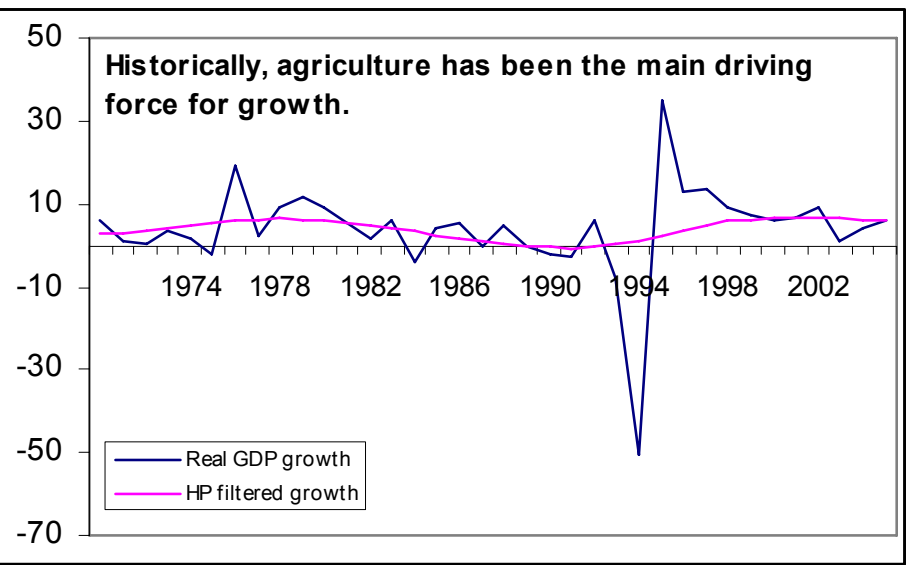
of capacity constraints. Given the importance of agriculture, staff urged the authorities to draw up a strategy in time for the 2008 budget.

\footnotetext{
${ }^{2}$ Http://www.earthinstitute.columbia.edu/mvp/locations/mayange/index.html.
} 
7. The energy supply is expensive and limited. Costly diesel generators provide more than half of Rwanda's electricity and tariffs are the highest in the region. Businesses also suffer from frequent outages beyond the very limited supply. The Lake Kivu project ${ }^{3}$ and a heavy-fuel-oil generator are expected to replace diesel power; and the authorities are also building new hydropower plants and implementing strategies to preserve alternative energy sources, such as wood fuel. Staff welcomed these activities and expected that they would mitigate pressures on the medium-term current account by reducing oil imports.

8. Transportation links are minimal. High transportation costs and slow routes both internally and through Tanzania and Kenya severely constrain Rwanda's access to markets, though the domestic road network is being improved and Rwanda is expected to benefit from regional efforts to rehabilitate key corridors, such as the World Bank's East Africa Trade and Transport Facilitation project. Nonetheless, some officials felt that Rwanda should give more attention to developing its Information and Communications Technology and services sectors to circumvent transportation constraints.

9. The financial system is shallow and the savings rate is low. Though banking sector indicators have improved and the authorities are reinforcing banking supervision guided by the 2005 Financial Sector Assessment Plan (FSAP) (Table 8), the financial system is still dominated by a small number of banks and credit is concentrated in a few sectors. The FSAP's medium-term recommendations directed at increasing Rwanda's savings rate and opening up access to credit are being fleshed out, and staff welcomed the presentation of a detailed agenda to donors in early 2007

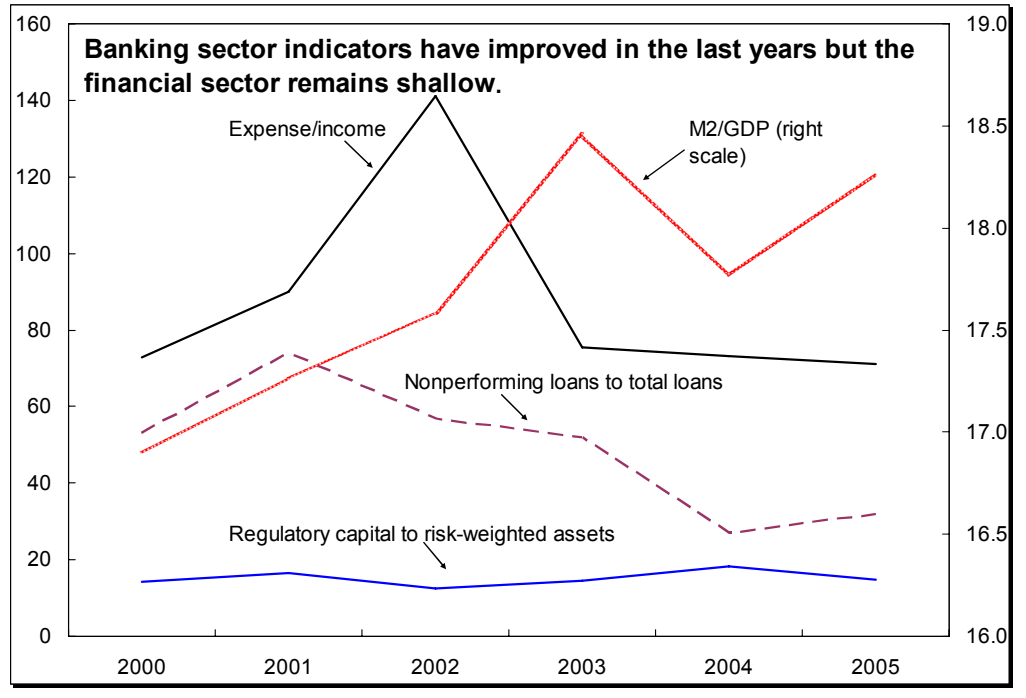
( $\mid 28$, MEFP). It also endorsed the intention of the National Bank of Rwanda (NBR) to strengthen supervision of microfinance institutions, ${ }^{4}$ introduce an Anti Money

\footnotetext{
${ }^{3}$ To generate electricity, the project would extract a methane gas layer dissolved in the deep waters of Lake Kivu.

${ }^{4}$ The NBR closed nine institutions in June when depositors started to withdraw funds due to mismanagement. To avoid contagion, depositors were reimbursed by the budget for half their net deposits (about 0.1 percent of GDP).
} 
Laundering/Combating the Financing of Terrorism law, and address weaknesses in the payments system and accounting standards.

10. Doing business is expensive. Rwanda scores high on ease of starting a business and enforcing contracts, but it lags behind in almost all other categories of the World Bank's cost of doing business report. ${ }^{5}$ The authorities have appointed a task force to address these weaknesses and also expected the business environment to improve because the legal system is being overhauled and a land law implemented ( $\$ 29$, MEFP). Private sector representatives complained about the tax burden and sometimes heavy-handed enforcement. While acknowledging that the tax system is generally sound, staff emphasized that the tax administration's onus should be on widening the tax net to the informal sector and ensuring that tax laws are applied evenly and fairly. It welcomed the increase in the revenue ratio in recent years, but emphasized that the planned export processing zone (to be financed by an AfDB grant) should be ringfenced to prevent tax leakage.

11. There is a shortage of qualified staff. Private sector representatives underscored that the small pool of skilled labor is a major bottleneck; they echoed the authorities' view (and the findings of the Ex Post Assessment) that capacity constraints hamper progress. While the authorities felt that the labor problem should be addressed by boosting tertiary education, donors advocated for a focus on primary and secondary education. Staff suggested that the on-the-job training for public accountants could serve as a model to build expertise in other areas.

12. Prioritizing policies will be a challenge. Some officials and private sector representatives felt that Rwanda's infrastructure gap should be tackled first. Other officials and some donors argued that the social and the agricultural sectors should have priority to reduce poverty and ensure food security and social peace. Staff stressed that further research was needed on how different policies affect growth and poverty. It also noted the need to assess the macroeconomic implications of spending to gauge whether it would give rise to pressures for an appreciation of the real effective exchange rate (REER) ( $\mid 16)$.

\section{B. Enhancing Competitiveness}

13. Trade integration is fundamental to Rwanda's development strategy. Involving subsistence farmers in trade could significantly reduce poverty and help diversify the economy, and broadening Rwanda's small export base would enhance its capacity to carry external debt and finance development.

\footnotetext{
${ }^{5}$ Rwanda placed 158 (of 175 countries) in both the 2006 and 2007 World Bank Doing Business reports.
} 
14. Improvements in competitiveness will depend on structural reforms. While the REER is considerably more depreciated than before the genocide, it has started to appreciate. Because the fact that exports did not respond to the depreciation suggests that nonprice barriers are the main obstacle to trade, trade would be best fostered through sectoral export promotion strategies ( $\$ 27$, MEFP) and the removal of structural bottlenecks. Authorities and staff considered the current level of the REER to be broadly in line with fundamentals and not a problem for competitiveness. There was also consensus that macro policies had to be geared to mitigating pressures for further appreciation that might arise from a scaling up ( $₫ 18)$.
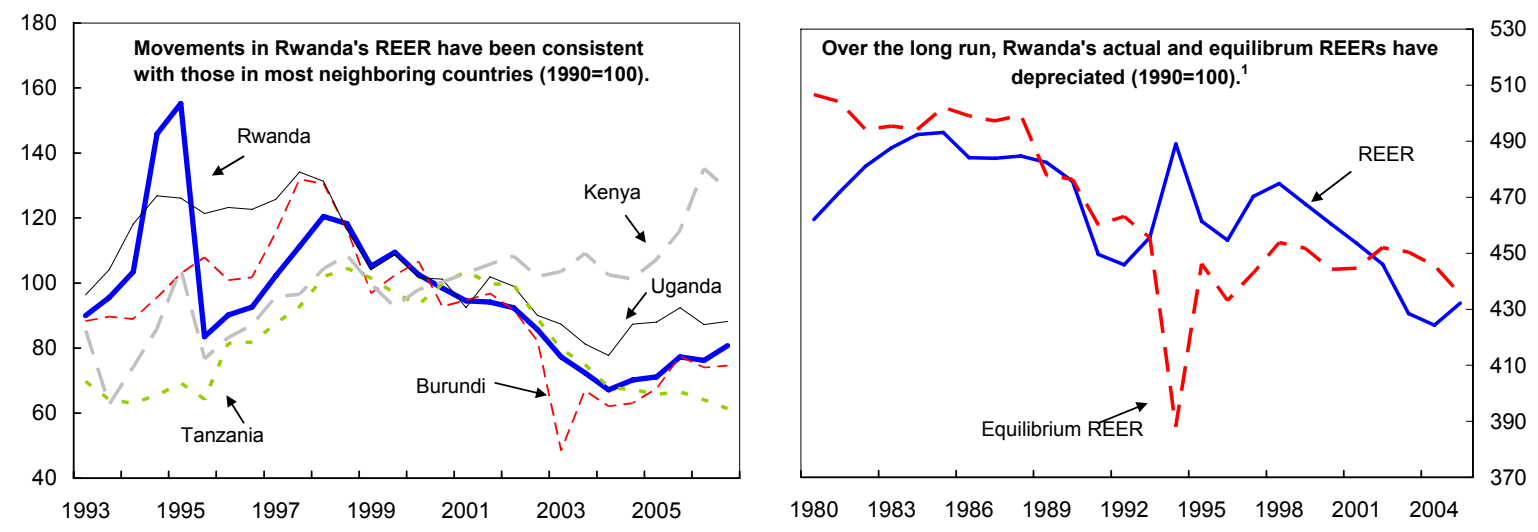

Source: INS and IMF staff estimates.

${ }^{1}$ Preliminary estimates of the equilibrium REER based on IMF staff estimates.

15. Trade arrangements could further boost Rwanda's exports. Rwanda's joining the EAC is commendable; staff welcomed the reduction in Rwanda's average tariffs resulting from the adoption of EAC's common external tariff and emphasized the scope membership offers for leveraging improvements in regional infrastructure and the business climate ( $\mid 25$, MEFP). In response to staff concerns that higher EAC tariffs on some food items could adversely affect the poor, the authorities said they would raise this, as well as possible revenue losses and the protection of some industries, in the negotiations for a customs union. They also noted a possible conflict between a customs union with the EAC scheduled for 2008 and COMESA scheduled for 2009 (Rwanda is a COMESA member). While the authorities are not pursuing bilateral trade arrangements, they are progressing toward an Economic Partnership Agreement with the European Union. Staff recommended that Rwanda also make more use of its eligibility for the U.S. African Growth and Opportunity Act.

\section{Boosting Growth-A Scenario for Scaling-Up}

16. Scaled-up aid would accelerate Rwanda's progress toward the MDGs. The financing needs are massive: while the full costs of reaching the MDGs are not known, the 
World Bank puts the price for achieving the health and education goals at more than 5 percent of GDP per year. Budget support in 2007 will be higher than before (by at least 1 percent of GDP) from the AfDB, the Fast Track Education Initiative, and two donors (the Netherlands and Germany), who had, so far, provided only project support. Further scaling up is possible in the medium term through, e.g., the U.S. Millennium Challenge Account. However, the order of magnitude and timing of scaling up are uncertain.

17. Scaled-up aid would also bring macroeconomic challenges. Spending the scaledup aid generates an increase in demand, which would put upward pressure on prices and the real exchange rate. Inflationary pressures would be mitigated to the extent that some of the higher spending was on imports or if there were a supply response in the economy. To best deal with these pressures, Rwanda would thus need to raise absorption of imports by allowing the nominal exchange rate to appreciate, and reduce obstacles to growth. Staff highlighted the challenges through an illustrative scenario (Box 2). The authorities thought the scenario would provide useful guidance for the PRSP update. Staff stressed that dialogue with stakeholders is necessary to generate ownership and ensure that policies achieve their intended purpose, and that budgets should be embedded in a medium-term framework.

\section{More flexibility in the exchange rate is crucial if Rwanda is to absorb more aid} and manage a likely real appreciation. While the NBR is committed to a flexible exchange rate, the exchange rate has in fact behaved like a conventional fixed peg. Staff reiterated that a peg was not the best exchange regime, particularly if aid is scaled up. To avoid rekindling inflation or costly sterilization through domestic debt, ${ }^{6}$ domestic spending should be sterilized predominantly by the sale of foreign exchange to raise absorption. Staff noted that a gradual approach in allowing a nominal appreciation was appropriate, given that exports would be adversely affected by exchange rate volatility. However, it saw a need for Rwanda to accelerate foreign exchange sales to prevent a build up of domestic debt. While agreeing that the exchange rate should be more flexible, the authorities expressed concern about the possibility that the appreciation of the exchange rate might reverse if aid inflows slowed. Staff responded that strong policy implementation would lessen the risk of a reversal of aid flows.

\footnotetext{
${ }^{6}$ Defined as consolidated debt of the government and the NBR (NBR papers and deposit facility).
} 


\section{Box 2. Scaled-Up Macroeconomic Framework}

The scenario outlined in the selected issues paper assumes that growth would be boosted to $81 / 2$ percent, halving poverty by 2020 . It highlights how the main macroeconomic challenges of scaling up can be addressed:

- $\quad$ To gradually increase the demand for domestic goods and services shift the composition of expenditures. Initially, the impact of aid flows on the real exchange rate would be mitigated by the need to front-load public investment (which is import-intensive) to address Rwanda's infrastructure gap. Over the medium term, capital spending would be gradually reduced to make room for higher recurrent social spending, which would correspondingly increase domestic demand.

- $\quad$ To keep debt sustainable finance the development effort mostly through grants.

- $\quad$ To move beyond aid dependence gradually raise revenue, invest in human capital, and increase domestic savings.
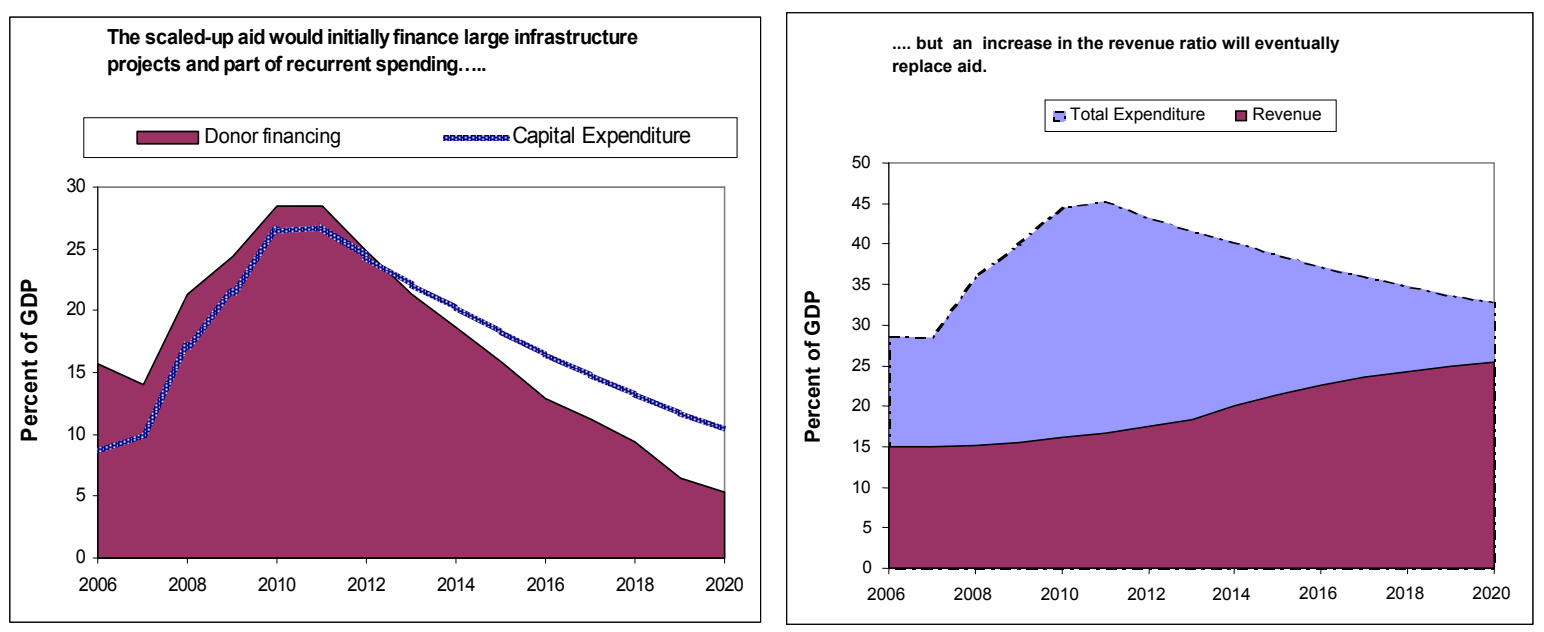

19. Delivery of public services will also have to be strengthened.

- $\quad$ Public expenditure management. To implement the Organic Budget Law, the authorities have created an action matrix ( $(18$, MEFP). While the matrix is a step in the right direction, staff urged the authorities to tackle current weaknesses more systematically, such as looking at their interaction with other reforms, including decentralization. The decentralization process in turn warranted close monitoring. 
- $\quad$ Civil service reform. The authorities recognized the need to resist wage pressures stemming from wage increases for the core civil service in $2006{ }^{7}$ Staff regretted the delay in the wage review (end-2006 benchmark), which is important to guide wage policy in the medium term ( $\$ 16$, MEFP). It acknowledged that some pay increase (possibly hinging on performance contracts) might be needed to retain qualified staff. Though it would be justifiable to increase the wage bill to hire more teachers or health workers, staff cautioned about using a scaling up to raise the wages of current public employees.

\section{Program Issues}

\section{The PRGF arrangement covering 2006-09 is designed to address Rwanda's medium-term challenges.}

\section{A. On Track, But There Are Pressures}

21. Though macroeconomic performance is aligned with the program, short-term risks have evolved on the monetary side.

- $\quad$ Growth is expected to exceed 4 percent despite a weak harvest because activity is buoyant in the manufacturing, services, and financial sectors. However, reflecting mostly a rise in food and energy prices, 12-month inflation gradually increased to 11 percent in November.

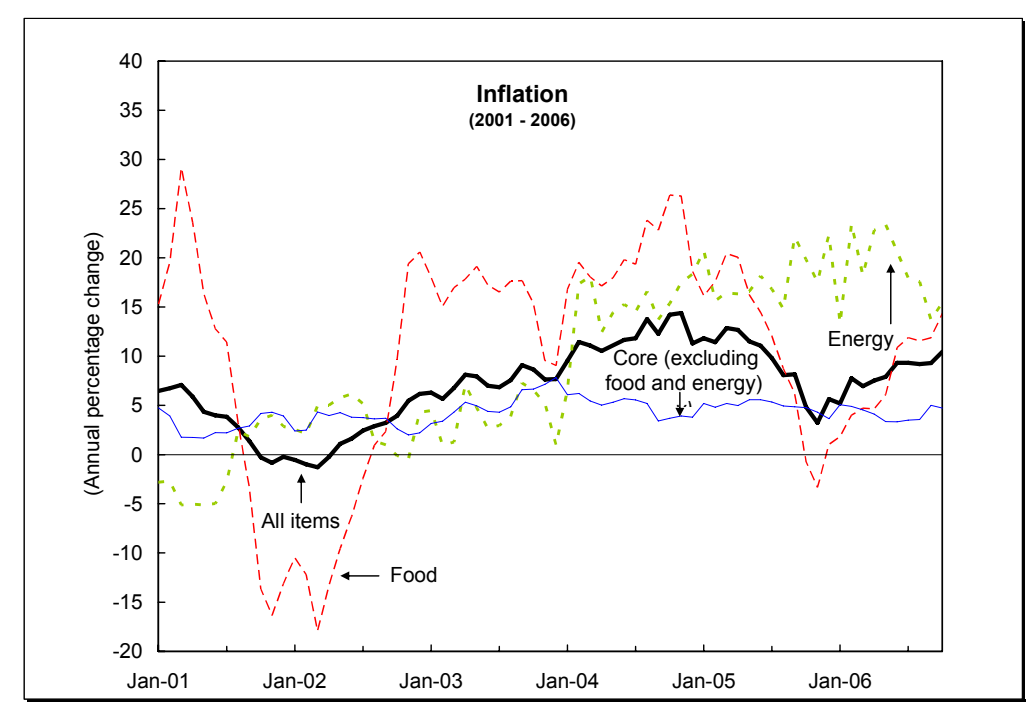

- $\quad$ Fiscal policy was tighter than programmed by about 0.75 percent of GDP in the first half of 2006, because revenue overperformed due to the inclusion of fringe benefits in the income tax. While expenditures were on track, their import component was less

\footnotetext{
${ }^{7}$ The wage increases for the core civil service were largely neutral on the wage bill as accompanied by retrenchment.
} 
than expected. Moreover, delay in the Lake Kivu project meant that priority spending was slightly below target. ${ }^{8}$ All end-September targets were met.

- $\quad$ Reserve money was within the end-June and end-September program limits, but

- Management of monetary policy was complicated by a rapid expansion in private sector credit (by 26 percent year-on-year in September) and broad money. While most of the increase appears to be demand-driven, some banks may be lowering their lending standards.

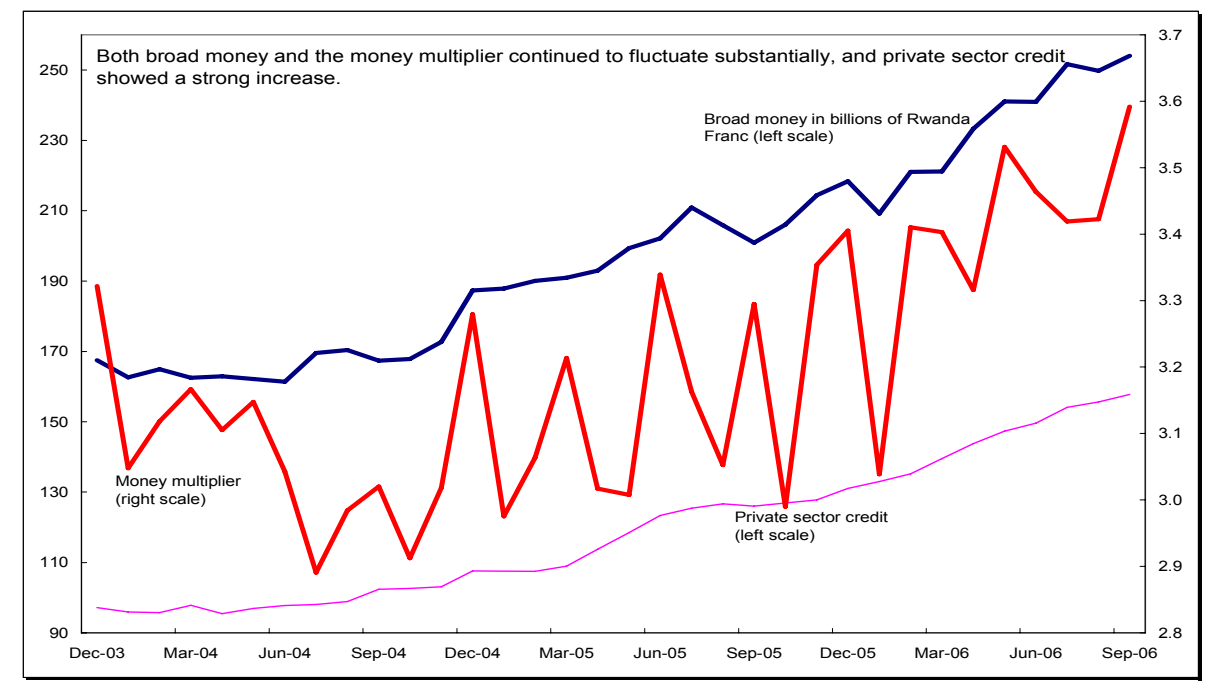

- Net international reserves exceeded the targets primarily because foreign exchange sales were lower than programmed, tracking lower foreign exchange demand from the budget. To keep reserve money within the program limits, the NBR resorted to sterilization using domestic instruments-implying an increase in domestic debt through September compared with year-end 2005. At the same time, the REER continued to appreciate (5 percent in the first three quarters of 2006).

\footnotetext{
${ }^{8}$ Without this technical delay, the end-June target for priority spending would have been met. The delay has been resolved, but there may be further delays because the project relies on a unique, not yet fully proven technology.
} 


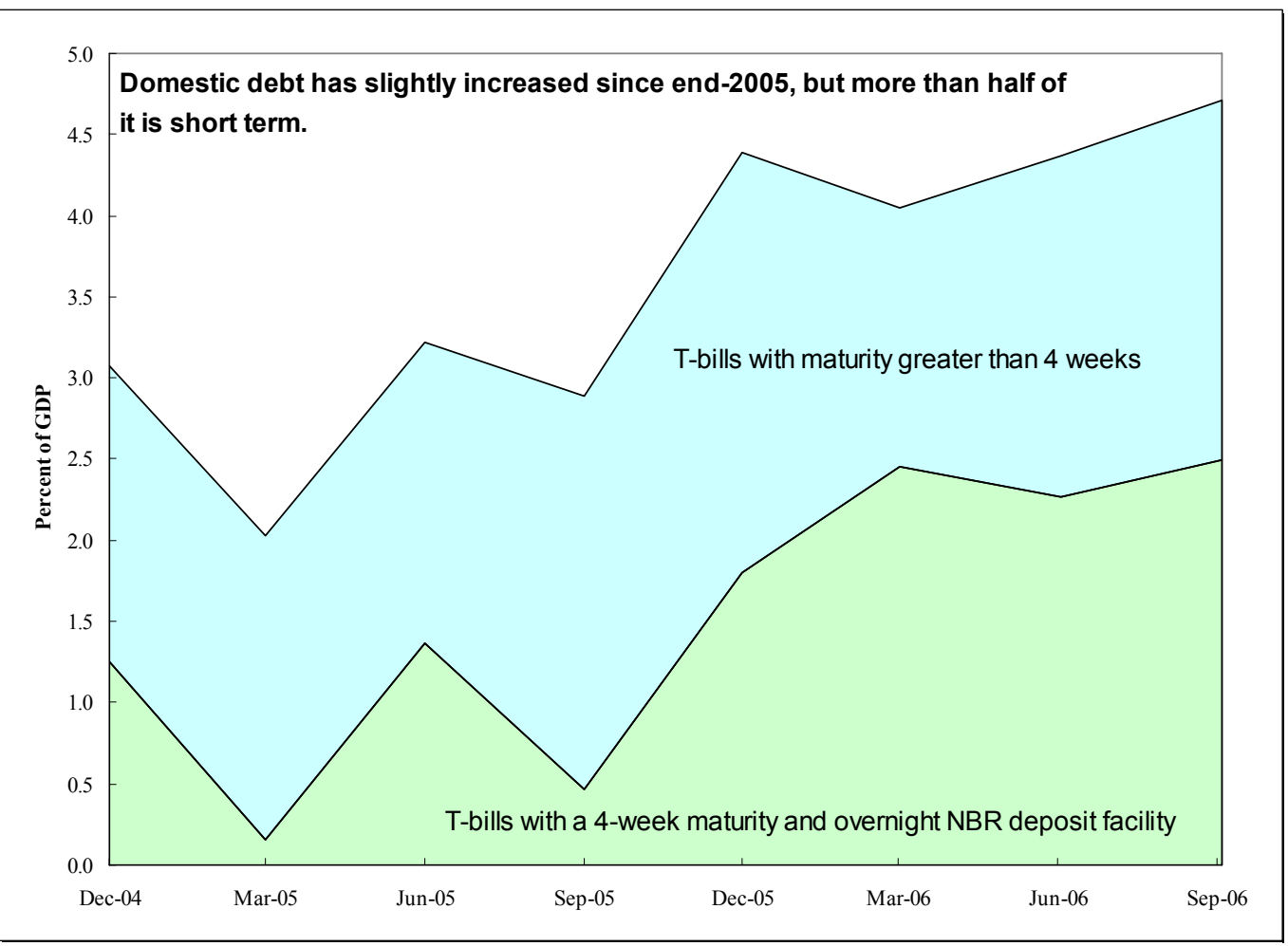

22. All but two performance criteria (PCs) for the first review have been met. All quantitative end-June PCs were met except for the floor on priority spending. On the structural side, the September PC on submission of the banking law and three benchmarks (on public expenditure management, debt management, and private sector development) were met. The second element of the August PC on monitoring project accounts has been implemented as a prior action (Table 7 and MEFP Table 3). As a long-term solution to the management of the state-owned Prime Holdings company's two hotels, a 30-year lease contract was signed with an international hotel chain.

\section{B. The Medium-Term Framework}

\section{Given uncertainties related to scaling up, the medium-term macroeconomic} framework takes a cautious view about aid. Aligned with the one in the previous staff report (Country Report No. 06/245), it envisages a real growth rate of 4-5 percent, inflation of 5 percent, and international reserves of at least 4 months of imports. While the authorities expected real growth to exceed the program levels, they felt that the program should be anchored on prudent assumptions to minimize policy mistakes; adjustments could be made during a review. In light of the risks of weather-related shocks, the program will continue to accommodate food imports in case there is an emergency. 
Rwanda: Medium-Term Framework, 2003-09

\begin{tabular}{|c|c|c|c|c|c|c|c|}
\hline & $\begin{array}{r}2003 \\
\text { Act. }\end{array}$ & $\begin{array}{r}2004 \\
\text { Act. }\end{array}$ & $\begin{array}{r}2005 \\
\text { Act. }\end{array}$ & $\begin{array}{c}2006 \\
\text { Proj. }\end{array}$ & $\begin{array}{l}2007 \\
\text { Proj. }\end{array}$ & $\begin{array}{c}2008 \\
\text { Proj. }\end{array}$ & $\begin{array}{l}2009 \\
\text { Proj. }\end{array}$ \\
\hline \multicolumn{8}{|l|}{ Economic growth and inflation (percentage change) } \\
\hline Real GDP (percentage change) & 0.9 & 4.0 & 6.0 & 4.2 & 4.5 & 4.7 & 4.9 \\
\hline Real GDP (per capita) & -0.7 & 2.5 & 4.2 & 2.4 & 2.7 & 2.8 & 3.0 \\
\hline Consumer price index (eop) & 7.7 & 10.2 & 5.6 & 5.0 & 5.0 & 5.0 & 5.0 \\
\hline \multicolumn{8}{|l|}{ Central government budget (percent of GDP) } \\
\hline Revenue & 13.5 & 13.9 & 15.1 & 15.0 & 15.0 & 15.2 & 15.4 \\
\hline Grants & 8.1 & 11.9 & 14.1 & 13.4 & 12.5 & 13.1 & 12.9 \\
\hline Government expenditure and net lending & 23.9 & 26.1 & 28.5 & 28.6 & 29.7 & 29.4 & 29.5 \\
\hline Current expenditure & 17.8 & 15.9 & 18.0 & 19.3 & 19.4 & 19.2 & 19.2 \\
\hline Capital expenditure & 5.6 & 8.5 & 10.1 & 8.5 & 9.6 & 9.6 & 9.6 \\
\hline Domestic fiscal balance (excl. demobilization spending) & -4.7 & -5.2 & -4.3 & -5.6 & -6.8 & -6.8 & -6.7 \\
\hline \multicolumn{8}{|l|}{ Overall balance (payment order) } \\
\hline After grants & -2.3 & -0.2 & 0.7 & -0.2 & -2.1 & -1.1 & -1.1 \\
\hline Before grants & -10.3 & -12.1 & -13.4 & -13.6 & -14.7 & -14.3 & -14.0 \\
\hline \multicolumn{8}{|l|}{ National accounts (percent of GDP) } \\
\hline Gross domestic investment & 18.4 & 20.5 & 22.6 & 21.3 & 23.0 & 22.5 & 22.6 \\
\hline Of which: private & 12.8 & 12.0 & 12.5 & 12.8 & 13.4 & 12.9 & 13.0 \\
\hline Gross national savings & -0.8 & 2.3 & 3.2 & 2.2 & 2.0 & 3.4 & 4.3 \\
\hline Current account bal. (excl. grants) & -19.2 & -18.2 & -19.4 & -19.1 & -21.1 & -19.1 & -18.3 \\
\hline \multicolumn{8}{|l|}{ Balance of payments (in millions of U.S. dollars) } \\
\hline Exports of goods and services & 139.5 & 189.7 & 227.9 & 264.6 & 280.3 & 304.9 & 322.1 \\
\hline Imports of goods and services & 463.9 & 524.1 & 666.6 & 761.3 & 896.9 & 946.6 & 1012.3 \\
\hline Current account balance (incl. grants) & -131.1 & -55.5 & -66.5 & -201.8 & -260.8 & -231.3 & -239.2 \\
\hline Overall balance & -33.6 & 90.2 & 111.2 & 35.3 & -55.4 & 25.5 & 49.6 \\
\hline Gross official reserves (months of imports of G\&S) & 5.0 & 5.7 & 6.4 & 4.9 & 4.3 & 4.4 & 4.5 \\
\hline
\end{tabular}

Sources: Rwandan authorities; and IMF staff estimates and projections.

\section{Macroeconomic Policies}

\section{Preserving macroeconomic stability while accommodating scaled-up aid is the} primary macroeconomic challenge. With indications for a real appreciation already appearing in 2006, managing the domestic demand impact of fiscal policies will become all the more important with the scaling up expected in 2007.

\section{Program for 2006}

25. Despite additional spending, the fiscal targets are achievable. The authorities plan to spend an additional 0.4 percent of GDP in 2006 on the interest bill, replenishing fuel stocks, and reimbursing depositors in the closed microfinance institutions. These items would be more than covered by the revenue overperformance. However, if the African Union (AU) continues to delay reimbursing Rwanda for peacekeeping efforts in Darfur, the net domestic financing target for 2006 might be missed. 
26. Risks to the monetary program will need to be reduced by

- Keeping the monetary stance tight. Staff welcomed the NBR's decision not to loosen monetary policy despite an upward revision in growth. The recent inspections of commercial banks that had substantially increased credit was timely and should be continued to monitor the quality of banks' portfolio. However, staff expressed concern about the NBR working around the monetary program by allowing reserve money to exceed end-quarter targets during the quarter. It urged the authorities to mop up the liquidity so as to not further fuel private sector credit. Staff also felt that the 2006 inflation target of 5 percent was not likely to be met. The authorities agreed, but they expect inflation to decline in the coming months. They were not overly concerned with excess liquidity during the quarter and the increase in private sector credit, given strengthened banking supervision. Should inflationary pressures persist, they are ready to tighten their stance, and also agreed to reduce excess liquidity during the quarter. In the meantime, developments in inflation and private sector credit would be closely monitored in coordination with staff.

- $\quad$ Following through with the agreed intervention strategy. Should the foreign exchange demand from the budget stay low, staff expressed concern about possible costly sterilization operations to mop up the liquidity from higher domestic fiscal spending. Thus, with continuing pressures for a real exchange rate appreciation, absorption should be increased by stepping up foreign exchange sales. At the same time, better analysis of the import component of the fiscal program would facilitate monetary management. The authorities responded that they are trying to increase the import component of the budget and improve its measurement, and felt that changes to the auction system ( $(21$, MEFP) would stimulate foreign exchange sales. Some officials also thought that commercial banks were resisting an appreciation, because they had net open positions. Staff recommended setting up a forum for discussion with banks to overcome their resistance.

\section{Program for 2007}

\section{The program contains a safeguard to manage a possible further real}

appreciation from higher budget spending. Acknowledging large development needs, the program allows for an increase in the deficit (excluding spending on peacekeeping and demobilization) by about 1 percent of GDP. This increase is primarily for investment and is partially financed by a drawdown of reserves, given the comfortable reserve position. Grants are likely to be higher than programmed. However, the program would accommodate further expansion in the deficit only after a review establishes that the expansion would not lead to 
a rekindling of inflation or an undue increase in domestic debt. ${ }^{9}$ Staff cautioned that, without medium-term scaling up, the increase in spending would be one off and there could be a need for fiscal adjustment in 2008. Financing the budget by drawing down reserves is not sustainable.

\section{This safeguard is also important because the authorities plan to accelerate} project spending. Project spending is not included in the definition of the domestic deficit and thus not limited by the program (unless debt-financed, 931 ) because it is difficult to monitor. Although projects generally have a relatively high import component, accelerated spending could exacerbate pressures for a real appreciation. This is particularly important as unused project disbursements of almost 5 percent of GDP were accumulated by endSeptember $2006,{ }^{10}$ likely reflecting absorptive constraints. The program envisages some draw down of project deposits during 2007, but, to better gauge spending pressures, staff welcomed an analysis of what had prevented the disbursement from being spent ( $\$ 17$, MEFP). It also urged the authorities to integrate project spending into future budgets.

\section{Budget}

29. The authorities will keep the revenue ratio at its 2006 level despite some revenue losses ( $₫ 13$, MEFP). Several measures are planned to offset losses from reducing the corporate tax rate and abolishing a warehouse fee (applicable to all imports). The latter was particularly welcome because the fee had worked as a hidden tariff. Staff commented that there was further scope for raising petroleum excises, given that pump prices were lower in Rwanda than in Burundi and Uganda.

30. Discussions on the 2007 budget focused on expenditure composition ( $\$ 14$, MEFP for a more detailed presentation). Staff stressed that it would have been easier to assess the budget if it had been embedded into a medium-term expenditure framework.

- $\quad$ Priority spending ${ }^{11}$ will increase by at least $1 \frac{1}{2}$ percent of GDP, directed to enhancing access to water and energy, improving the quality of teaching to address low school completion rates, and establishing land offices to implement the new land law (see Box 3).

\footnotetext{
${ }^{9}$ Additional spending of about 2 percent of GDP is already identified for education under the Fast Track Initiative (1 percent of GDP) and for so called "contingent spending," which would be allocated in equal proportion to priorities and nonpriorities.

${ }^{10}$ This accumulation took place over several years.

${ }^{11}$ Development priorities as identified in the PRSP; "nonpriority" spending is the residual, but nonetheless essential for the provision of basic public and social services.
} 
- "Nonpriority" spending would decline slightly compared to 2006, reflecting mostly across-the-board restraint. This would allow for new spending in such areas as social cohesion (courts for those accused of genocide, refugee repatriation, and prison feeding); and administrative matters (opening embassies in Sudan and the Netherlands, issuing new identity cards, setting up arbitration courts and the accountant general's office, and rehabilitating laboratories for quality control). If there is a further expansion in the deficit, nonpriority spending would increase across the board.

\section{Box 3. Use of MDRI Relief}

MDRI relief resulted in a reduction in Rwanda's debt by more than US\$1 billion. However, except for the relief from the IMF, the net gain with lower disbursements financing part of the relief is expected to be small.

For 2007 the authorities will allocate the MDRI relief to the water and energy sectors.

Debt Relief Under MDRI

\begin{tabular}{|c|c|c|c|c|c|}
\hline & \multirow[t]{2}{*}{ Stock } & \multicolumn{4}{|c|}{ Flows } \\
\hline & & 2006 & 2007 & 2008 & 2009 \\
\hline IMF & 76.6 & 7.7 & 8.0 & 4.9 & 4.2 \\
\hline World Bank $^{1}$ & 883.9 & 2.1 & 2.5 & 2.7 & 2.8 \\
\hline $\mathrm{AfDF}^{1}$ & 266.1 & 3.3 & 3.4 & 3.6 & 3.8 \\
\hline Total & 1226.6 & 13.1 & 13.9 & 11.2 & 10.8 \\
\hline Total (\% of GDP) & 51.2 & 0.5 & 0.6 & 0.5 & 0.5 \\
\hline
\end{tabular}

31. Specific major expenditure items $(₫ 15, \mathrm{MEFP})$ :

- $\quad$ Electricity subsidy. Because electricity tariffs are the highest in the region and a further increase could stifle economic activity, the budget specifies a temporary subsidy of about 0.5 percent of GDP until a more efficient generator becomes operational (expected by the end of 2007).

- $\quad$ Military spending. While military spending is expected to decline in 2007 to 2.4 percent of GDP, there was an unprogrammed increase in 2006 (to 2.5 percent of GDP from 2.3 percent of GDP in 2004), when wages for conscripts were increased. 
The authorities decided not to increase wages for military professionals and officers in 2007 in response to donor concern.

- $\quad A U$ peacekeeping. The authorities intend to continue peacekeeping efforts in Darfur, but would recall their troops if reimbursements do not materialize. So far, outlays of about 0.7 percent of GDP have had to be financed from budgetary resources.

\section{Debt}

32. The authorities want to tightly limit debt financing. With IDA expected to provide grants in 2007 rather than loans and a sustainable pick up in export receipts in 2006, Rwanda's debt sustainability indicators are slightly more favorable than at the last review. Nonetheless, starting in 2014 the NPV of debt-to-exports ratio would exceed the 150 percent threshold. Given the vulnerabilities associated with a narrow export base, the authorities feel strongly about not exceeding the threshold and have crafted a borrowing strategy that would limit the NPV of debt-to-exports ratio to below 125 percent over the long term. Consistent with this intention, the program introduces new indicative targets on the net present value of external debt to limit the contracting of concessional loans. Staff welcomed the authorities' commitment to contain borrowing, but pointed out that staying within these limits would to a large extent depend on whether multilaterals, which account for most of Rwanda's aid, would continue to provide grants.

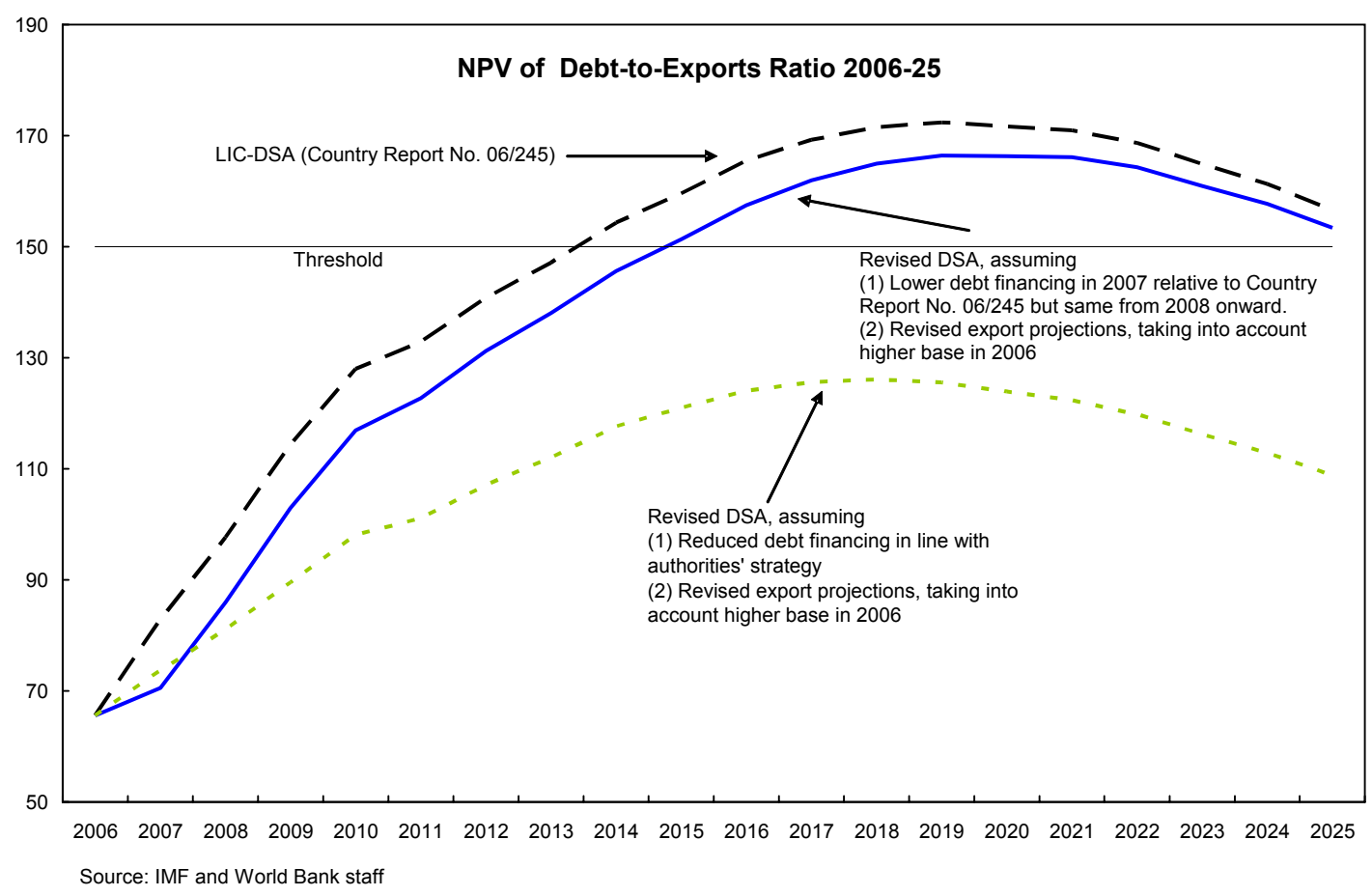




\section{Structural Reforms}

33. The focus of structural conditionality is broadly unchanged (Table 3, MEFP).

- $\quad$ Public expenditure management. Emphasis will be on the implementation of the Organic Budget Law and the reconciliation of bank statements.

- $\quad$ Poverty reduction. To better target policies, the authorities will formulate detailed community poverty profiles.

- Financial sector reform. The priority here is to strengthen NBR's supervision of microfinance institutions. The medium-term agenda will be discussed at a donors' meeting ( $(99)$.

- $\quad$ Private sector development. To improve property registration (which, through collateralization, is expected to improve access to financial services), the authorities will establish an office to register land titles.

\section{E. Data Issues}

34. Although economic data are generally adequate for surveillance, weaknesses hamper economic analysis. Weaknesses permeate all areas, but particular focus is needed on improving national accounts and price statistics, government finance, and balance of payments statistics.

\section{F. Program Risks and Monitoring}

35. Program risks have increased. The main risk is that the monetary program could go off track if the NBR resists a nominal exchange rate appreciation. Weak administrative capacity could delay structural reforms, and a new risk has emerged on the security front: Rwanda may have to cope with refugees from Tanzania, which has started to expel Rwandese, or from Burundi and the Democratic Republic of Congo, should the political situation deteriorate there. Finally, medium-term program funding could be affected by Rwanda recently breaking off its diplomatic relations with France in response to the accusations of a French judge that several high-ranking Rwandese officials were involved in the 1994 assassination of President Habyarimana.

36. Program monitoring for 2007 is described in the MEFP (qศ 930 and 31) and the technical memorandum of understanding. The program design is generally the same, but an indicative target on the NPV of external debt was added. 


\section{STAFF APPRAisal}

37. Rwanda is at a crossroads. The country has come a long way since the genocide: it has achieved macroeconomic stability, it is continuing with social rehabilitation, and it is making progress toward the MDGs. The challenge now is to make the best use of resources, including scaled-up aid, to boost long-term growth, which has been lackluster in recent years, and make inroads in poverty reduction.

38. Now that the major obstacles to growth are identified-low agricultural yields, severe infrastructure gap, an underdeveloped financial system, and high costs of doing business - the focus should move to prioritization. To help with sequencing reforms, staff encourages the authorities to engage in more dialogue with stakeholders and better research the link between policies and poverty reduction and growth. It also underscores the critical role of the agricultural strategy for food security and social cohesion.

39. With Rwanda benefiting from scaled-up aid, the centerpiece of the program will be management of the domestic demand impact of fiscal policies. A widening of the budget deficit and possible acceleration in project spending in 2007 could intensify pressures for real exchange rate appreciation that appeared in 2006. Thus, staff urges the authorities to step up sales of foreign exchange and allow more exchange rate flexibility so as to avoid costly domestic sterilization and a rekindling of inflation.

40. The 2007 budget is an important milestone in Rwanda's development. The expenditure composition is appropriate with increased allocations for priorities, particularly investments in the water and energy sectors, which are consistent with Rwanda's development objectives. Looking forward, staff urges the authorities to embed their policies in a medium-term expenditure framework.

41. Monetary policy must bring inflation back to single digits. The increase in private sector credit is generally encouraging but staff is concerned about inflation. Unless inflation is set solidly on a downward path, the authorities must keep the monetary stance tight and continue to scrutinize private sector credit.

42. A range of reforms is designed to improve public service delivery. The public expenditure management action plan is promising but reforms need to be considered in a broader context. Better monitoring of project accounts is especially important, given their macroeconomic relevance. The intention of the authorities to strengthen debt management and limit debt financing is commendable. The authorities should also enhance training to address capacity constraints. While tax administration has improved substantially, increases in the revenue ratio should come from widening the tax net to the informal sector.

43. The efforts to improve the investment climate are welcome. Recent steps to augment Rwanda's electricity supply, improve commercial bank soundness, and overhaul 
business-related laws are commendable, and EAC membership could leverage regional improvements in the business climate and infrastructure. These and sectoral export promotion strategies are expected to enhance Rwanda's competitiveness, but the authorities might be well-advised to make more use of trade arrangements. Staff looks forward to discussing with the authorities medium-term financial sector reforms.

44. The 2003 safeguards assessment has been updated and staff encourages the authorities to address remaining vulnerabilities.

45. Staff recommends that the requested waivers be granted-their nonobservance was temporary - and that the first review of the PRGF arrangement be completed.

46. Staff recommends that the next Article IV consultation with Rwanda be held in accordance with the decision on consultation cycles approved on July 15, 2002. 
Figure 1: Real and Nominal Effective Exchange Rate (1997 average=100; foreign currency per Rwandan franc)

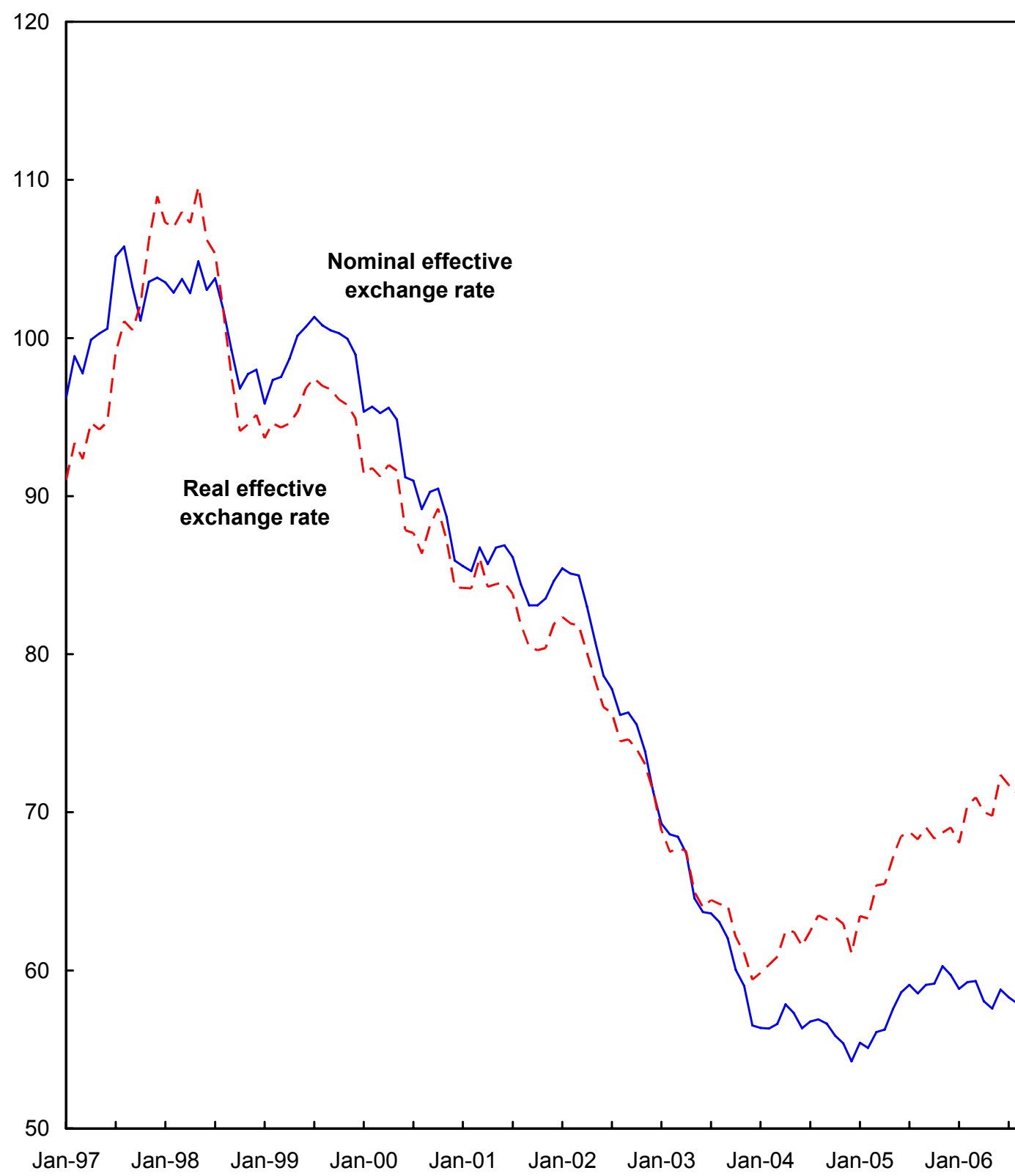

Source: INS and IMF staff estimates. 
Table 1: Rwanda: Selected Economic and Financial Indicators, 2003-09

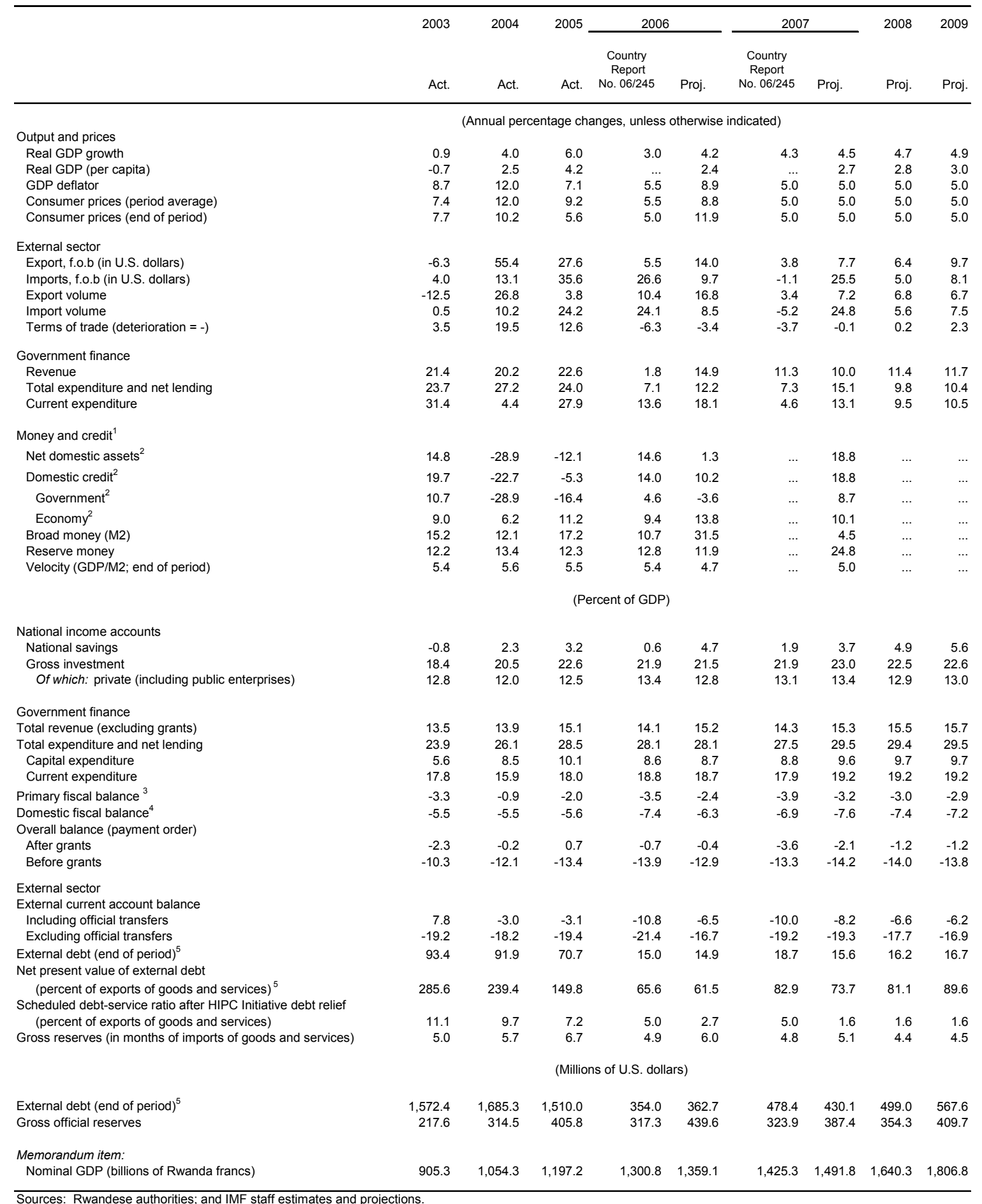

${ }^{1}$ Data up to 2005 based on current exchange rates; for 2006, based on program exchange rate of RF 553.7/US $\$$ and RF 549.9/US $\$$ for 2007.

${ }^{2}$ As a percent of the beginning-of-period stock of broad money.

${ }^{3}$ Revenue excluding grants minus current expenditure except interest due and exceptional expenditure minus domestically financed capital expenditure

${ }^{4}$ Revenue excluding grants minus current expenditure (excluding external interest) minus domestically financed capital expenditure and net lending

${ }^{5}$ After rescheduling, including arrears and new debt (the latter includes assumed project and budgetary disbursements for 2006-09) and based on assumptions about expected new borrowing.

\section{(C) International Monetary Fund. Not for Redistribution}


Table 2. Rwanda: Operations of the Central Government, 2005-09

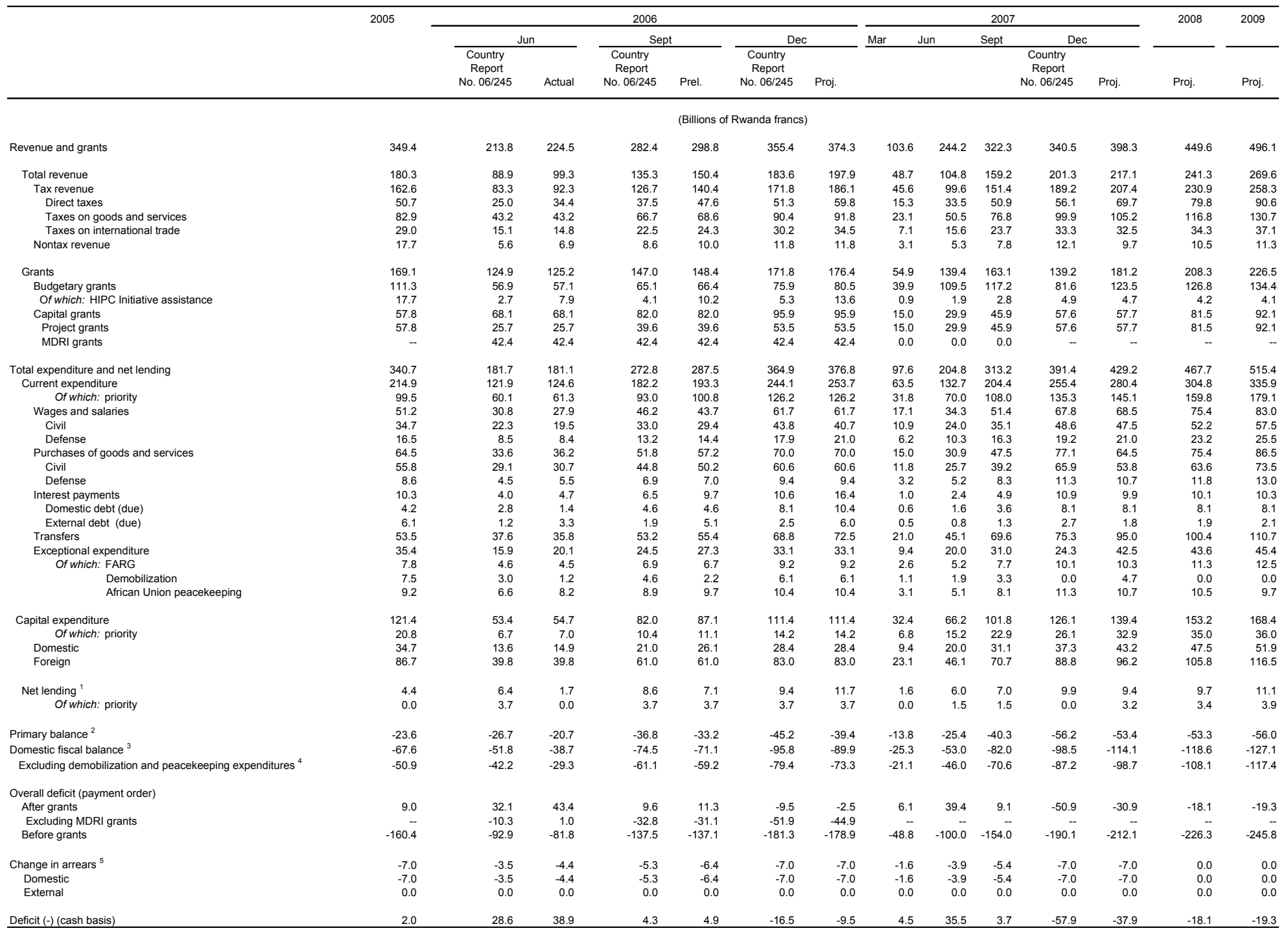


Table 2. Rwanda: Operations of the Central Government, 2005-09 (concluded)

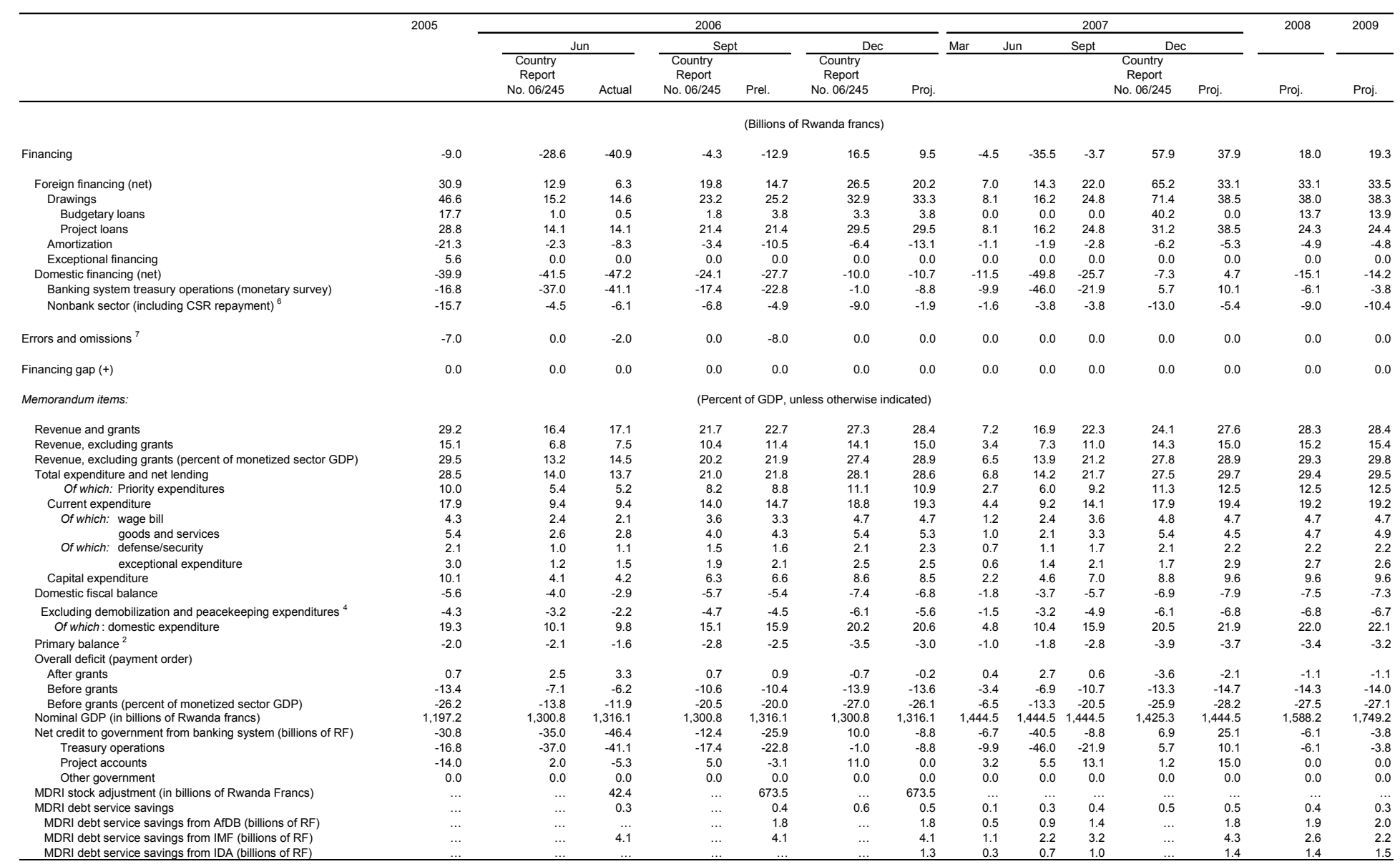

Sources: Rwandese authorities, and IMF staff estimates and projections

${ }^{1}$ Net lending in the fourth quarter of 2005 incorporates RF2.9 billion in receipts from the privatization of Rwandatel.

2 Defined as total revenue (excluding privatization proceeds) minus noninterest current expenditure minus domestically financed capital expendittre.

${ }^{3}$ Revenue excluding grants minus current expenditure, domestically financed capital expenditure, and net lending, excluding external interest.

${ }^{4}$ Actual data for 2005 exclude Rf8.5 billion expenditures for peacekeeping operations, which were not covered by grants. The domestic deficit excluding grant-financed peace keeping would have been 5 percent of GDP in 2005. For 2006, peacekeeping operations of up to Rf 7.3 billion might not be covered by grants.

${ }^{5}$ A negative sign indicates a reduction.
${ }^{6} \mathrm{CSR}=$ Caisse Sociale du Rwanda.

A negative number implies a discrepancy that is consistent with an underestimation of financing. 
Table 3. Rwanda: Monetary Survey, 2005-07

\begin{tabular}{|c|c|c|c|c|c|c|c|c|c|c|c|c|}
\hline & \multirow{3}{*}{$\begin{array}{l}2005 \\
\text { Act }^{1}\end{array}$} & \multicolumn{7}{|c|}{2006} & \multicolumn{4}{|c|}{2007} \\
\hline & & \multirow[t]{2}{*}{ Mar } & \multicolumn{2}{|c|}{ Jun } & \multicolumn{2}{|c|}{ Sep. } & \multicolumn{2}{|c|}{ Dec } & \multirow[t]{2}{*}{ Mar } & Jun & \multirow[t]{2}{*}{ Sept } & \multirow[t]{2}{*}{ Dec } \\
\hline & & & $\begin{array}{c}\text { Country } \\
\text { Report } \\
\text { No. } \\
06 / 245^{1}\end{array}$ & Act $^{1}$ & $\begin{array}{c}\text { Country } \\
\text { Report } \\
\text { No. } \\
06 / 245^{1}\end{array}$ & Prel $^{1}$ & $\begin{array}{c}\text { Country } \\
\text { Report } \\
\text { No. } \\
06 / 245^{1}\end{array}$ & $\operatorname{Proj}^{1}$ & & $\operatorname{Proi}^{1}$ & & \\
\hline Monetary authorities & \multicolumn{12}{|c|}{ (Billions of Rwanda francs) } \\
\hline Net foreign assets & 185.6 & 225.2 & 221.3 & 236.6 & 203.7 & 228.0 & 172.4 & 200.9 & 208.1 & 244.3 & 206.8 & 179.3 \\
\hline Foreign assets & 230.0 & 226.8 & 223.6 & 240.3 & 206.0 & 231.5 & 175.7 & 204.2 & 211.4 & 248.7 & 211.2 & 184.9 \\
\hline Foreign liabilities $^{2}$ & 44.4 & 1.6 & 2.3 & 3.7 & 2.3 & 3.5 & 3.3 & 3.3 & 3.3 & 4.4 & 4.4 & 5.6 \\
\hline Net domestic assets & -121.4 & -160.2 & -151.3 & -167.0 & -132.3 & -157.3 & -100.0 & -128.4 & -134.1 & -164.8 & -126.3 & -97.4 \\
\hline Domestic credit & -96.7 & -137.8 & -122.4 & -136.1 & -103.4 & -119.5 & -71.1 & -96.4 & -102.1 & -132.8 & -94.3 & -65.4 \\
\hline Government (net) & -60.5 & -105.3 & -99.6 & -98.3 & -79.1 & -77.1 & -51.1 & -69.9 & -76.6 & -110.4 & -78.7 & -44.8 \\
\hline Claims & 42.1 & 41.8 & 42.1 & 41.8 & 42.1 & 41.8 & 42.1 & 42.1 & 42.1 & 42.1 & 42.1 & 42.1 \\
\hline Of which: overdraft & -- & -- & -- & -- & -- & -- & -- & - & -- & -- & -- & -- \\
\hline Deposits (excluding autonomous bodies) ${ }^{2}$ & 102.6 & 147.1 & 141.8 & 140.1 & 121.2 & 118.9 & 93.2 & 112.0 & 118.7 & 152.6 & 120.9 & 86.9 \\
\hline Public nongovernment deposits (-) & -0.8 & -0.5 & -0.8 & -0.5 & -0.8 & -0.9 & -0.8 & -0.8 & -0.9 & -0.8 & -0.9 & -0.8 \\
\hline Nongovernment credit & -35.3 & -32.0 & -21.9 & -37.3 & -23.5 & -41.5 & -19.2 & -25.8 & -24.6 & -21.6 & -14.6 & -19.9 \\
\hline Private sector & 3.4 & 3.4 & 3.4 & 3.4 & 3.4 & 3.5 & 3.4 & 3.4 & 3.5 & 3.4 & 3.5 & 3.4 \\
\hline Public enterprises & 0.1 & -- & - & -- & 0.1 & -- & 0.1 & 0.1 & 0.1 & 0.1 & 0.1 & 0.1 \\
\hline Commercial banks & -38.9 & -35.5 & -25.4 & -40.7 & -27.0 & -45.0 & -22.7 & -29.3 & -28.2 & -25.2 & -18.2 & -23.4 \\
\hline Discount window & 1.4 & 1.3 & 1.4 & 1.3 & 1.4 & 1.2 & 1.4 & 1.4 & -0.6 & 0.4 & 1.4 & 1.4 \\
\hline Money market (- = absorption) & -40.2 & -36.8 & -26.8 & -42.0 & -28.4 & -46.2 & -24.1 & -30.7 & -27.6 & -25.6 & -19.6 & -24.8 \\
\hline Other items (net; asset + ) & -24.7 & -22.4 & -28.9 & -30.9 & -28.9 & -37.7 & -28.9 & -32.0 & -32.0 & -32.0 & -32.0 & -32.0 \\
\hline Reserve money ${ }^{3}$ & 64.2 & 65.0 & 70.0 & 69.6 & 71.4 & 70.7 & 72.4 & 72.4 & 74.0 & 79.5 & 80.5 & 81.9 \\
\hline Currency in circulation & 48.3 & 45.2 & 50.6 & 51.2 & 51.0 & 48.0 & 52.7 & 52.7 & 52.0 & 56.5 & 58.2 & 58.5 \\
\hline Commercial bank reserves & 13.7 & 17.1 & 16.7 & 16.1 & 18.2 & 19.7 & 17.5 & 17.5 & 19.7 & 20.8 & 20.1 & 21.1 \\
\hline Nonbank deposits & 2.2 & 2.7 & 2.7 & 2.2 & 2.2 & 3.0 & 2.2 & 2.2 & 2.2 & 2.2 & 2.2 & 2.2 \\
\hline Of which: autonomous public agencies & -- & 0.2 & -- & 0.3 & -- & 0.4 & -- & -- & -2.0 & -1.0 & -- & -- \\
\hline \multicolumn{13}{|l|}{ Commercial banks } \\
\hline Net foreign assets & 42.1 & 37.7 & 37.0 & 42.9 & 37.0 & 54.0 & 46.8 & 42.0 & 44.0 & 44.0 & 49.0 & 43.5 \\
\hline Foreign assets & 53.8 & 50.4 & 49.7 & 54.8 & 47.0 & 66.3 & 56.8 & 54.0 & 56.0 & 56.0 & 61.0 & 55.5 \\
\hline Foreign liabilities & 11.7 & 12.6 & 12.7 & 12.0 & 10.0 & 12.3 & 10.0 & 12.0 & 12.0 & 12.0 & 12.0 & 12.0 \\
\hline Reserves & 13.7 & 17.1 & 16.7 & 16.1 & 18.2 & 19.7 & 17.5 & 17.5 & 19.7 & 20.8 & 20.1 & 21.1 \\
\hline NBR deposits & 10.9 & 12.3 & 15.8 & 11.9 & 16.4 & 13.8 & 14.5 & 14.5 & 16.3 & 17.9 & 19.1 & 16.1 \\
\hline Required reserves & 14.9 & 15.7 & 15.1 & 17.2 & 15.9 & 18.2 & 16.8 & 17.4 & 17.8 & 18.4 & 18.6 & 19.1 \\
\hline Excess reserves & -4.0 & -3.4 & 0.7 & -5.4 & 0.5 & -4.4 & -2.3 & -3.0 & -1.5 & -0.5 & 0.5 & -3.0 \\
\hline Cash in vault & 2.8 & 4.8 & 4.8 & 4.2 & 2.8 & 5.9 & 3.0 & 3.0 & 3.0 & 3.0 & 3.0 & 5.0 \\
\hline Net credit from NBR (rediscount; liability -) & 38.9 & 35.5 & 25.4 & 40.7 & 27.0 & 45.0 & 22.7 & 29.3 & 28.2 & 25.2 & 18.2 & 23.4 \\
\hline Domestic credit & 128.7 & 131.5 & 143.1 & 138.8 & 150.5 & 145.3 & 149.9 & 159.6 & 163.4 & 173.7 & 178.8 & 184.3 \\
\hline Government (net) & -1.1 & -5.6 & 3.0 & -9.7 & 5.1 & -10.4 & -0.5 & -0.5 & -0.5 & -0.5 & -0.5 & -0.5 \\
\hline Credit & 21.1 & 14.8 & 21.1 & 12.8 & 21.1 & 12.8 & 21.1 & 21.1 & 21.1 & 21.1 & 21.1 & 21.1 \\
\hline Deposits & 22.2 & 20.4 & 18.1 & 22.5 & 16.1 & 23.1 & 21.6 & 21.6 & 21.6 & 21.6 & 21.6 & 21.6 \\
\hline Public enterprises & 2.1 & 0.9 & 0.9 & 2.3 & 2.1 & 1.4 & 2.1 & 2.1 & 2.1 & 2.1 & 2.1 & 2.1 \\
\hline Private sector & 127.6 & 136.1 & 139.2 & 146.2 & 143.3 & 154.3 & 148.2 & 158.0 & 161.7 & 172.0 & 177.1 & 182.7 \\
\hline Other items (net; asset + ) & -54.3 & -48.7 & -51.5 & -50.7 & -50.2 & -56.9 & -48.8 & -52.0 & -55.0 & -55.0 & -55.0 & -55.0 \\
\hline Deposits & 169.1 & 173.1 & 170.7 & 187.7 & 182.5 & 207.1 & 188.1 & 196.4 & 200.3 & 208.6 & 211.0 & 217.4 \\
\hline Private & 136.8 & 143.2 & 138.5 & 152.8 & 150.2 & 167.4 & 155.8 & 164.2 & 168.1 & 176.4 & 178.8 & 185.2 \\
\hline Public (nongovernment) & 32.2 & 29.8 & 32.2 & 34.9 & 32.2 & 39.7 & 32.2 & 32.2 & 32.2 & 32.2 & 32.2 & 32.2 \\
\hline
\end{tabular}

(C)International Monetary Fund. Not for Redistribution 
Table 3. Rwanda: Monetary Survey, 2005-07 (concluded)

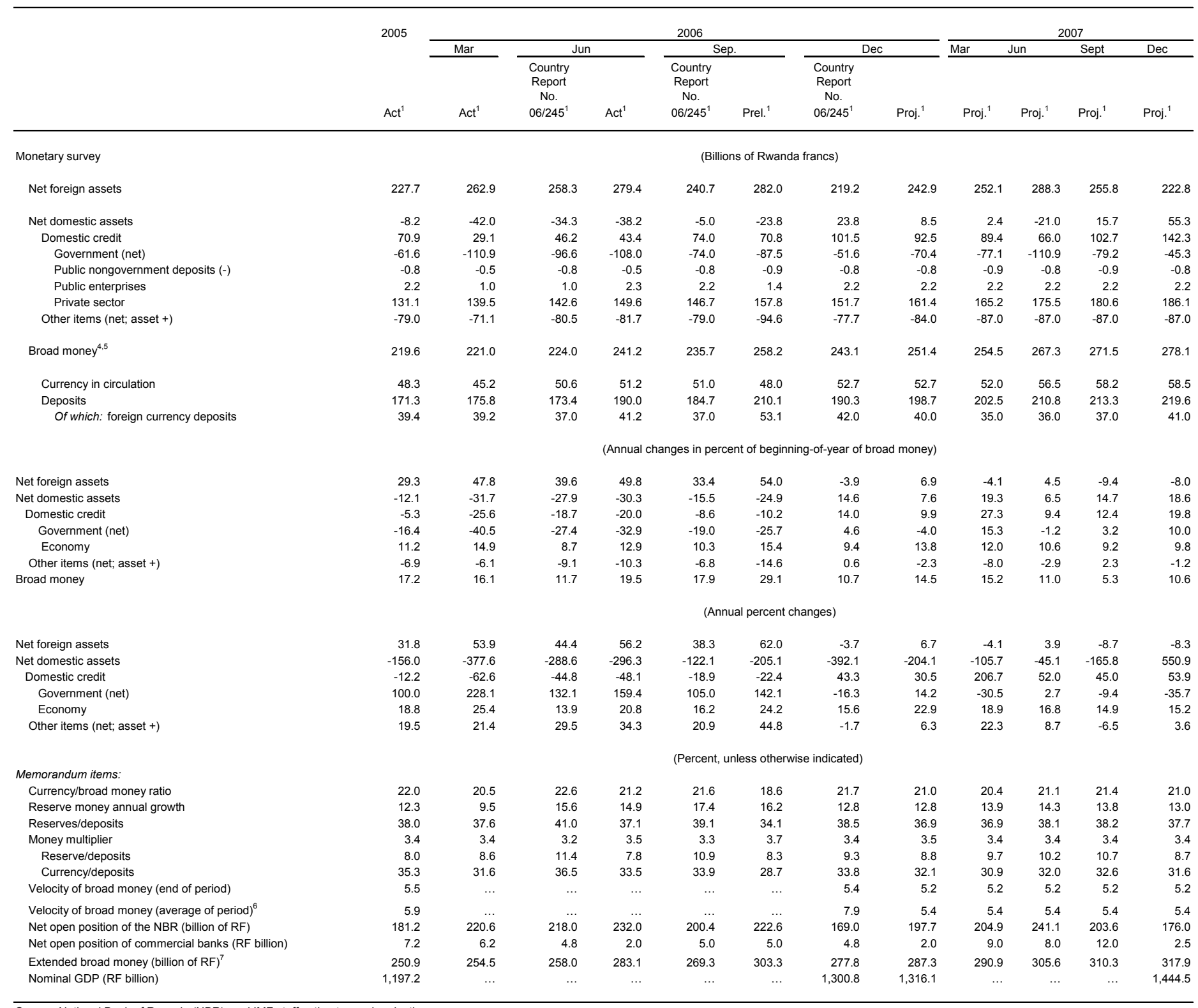

Source: National Bank of Rwanda (NBR); and IMF staff estimates and projections.

${ }^{1}$ Converted at the program exchange rate: for 2005: RF 566.9/US\$; for 2006: RF 553.7/US\$ and RF 549.9/US\$ for 2007.

${ }^{2}$ The IMF's MDRI reduced foreign liabilities at the NBR by RF 42.4 million with a counter entry in government deposits (in January 2006).

${ }^{3}$ The definition of reserve money as performance criterion or structural benchmark differs from the definition in the monetary program in that it excludes the deposits of a defunct savings bank, import deposits, and dormant accounts. It includes old notes demonetized at the end of 2004 but in circulation until the end of 2005. The PC and indicative targets refer to the last day of the period.

${ }^{4}$ From December 2005 onward, includes Caisse Hypothecaire du Rwanda (BHR) deposits (RF 1 billion at end-2005).

${ }^{5}$ End-September 2006 broad money includes RF 8 billion in foreign currency deposits for temporary repatriation of private investment, expected to unwind by December 2006.

${ }^{6}$ Data from 2005 onward revised to reflect corrections to the formula. Country Report 06/245 should have shown 5.6 for 2006.

${ }^{7}$ Broad money plus deposits in the Union de Banques Populaires de Rwanda (UBPR) and Rwanda Development Bank (BRD). 
Table 4. Rwanda: Balance of Payments, 2003-09

(Millions of U.S. dollars, unless otherwise indicated)

\begin{tabular}{|c|c|c|c|c|c|c|c|c|c|}
\hline & \multirow[t]{2}{*}{2003} & \multirow[t]{2}{*}{2004} & \multirow[t]{2}{*}{2005} & \multicolumn{2}{|c|}{2006} & \multicolumn{2}{|c|}{2007} & \multirow[t]{2}{*}{2008} & \multirow[t]{2}{*}{2009} \\
\hline & & & & $\begin{array}{c}\text { Country } \\
\text { Report } \\
\text { No. } 06 / 245\end{array}$ & Proj. & $\begin{array}{l}\text { Report } \\
\text { No. } \\
06 / 245\end{array}$ & Proj. & & \\
\hline Exports, f.o.b. & 63.0 & 97.9 & 125.0 & 131.9 & 142.5 & 136.9 & 153.4 & 163.3 & 179.2 \\
\hline Of which: coffee & 15.0 & 31.0 & 38.1 & 49.9 & 54.0 & 53.7 & 57.6 & 62.7 & 68.5 \\
\hline tea & 22.5 & 23.4 & 24.1 & 27.2 & 32.2 & 28.3 & 38.7 & 42.5 & 50.2 \\
\hline Imports, f.o.b. & 244.0 & 275.9 & 374.1 & 473.5 & 410.5 & 468.3 & 515.3 & 541.0 & 584.8 \\
\hline Trade balance & -181.0 & -178.0 & -249.1 & -341.6 & -268.0 & -331.4 & -361.9 & -377.8 & -405.6 \\
\hline Services (net) & -143.4 & -156.5 & -189.1 & -196.9 & -182.8 & -200.9 & -220.1 & -226.7 & -233.5 \\
\hline Income & -30.5 & -34.1 & -25.0 & -17.6 & -23.9 & -16.2 & -18.3 & -12.7 & -16.9 \\
\hline Of which: interest on public debt ${ }^{1}$ & -10.7 & -13.9 & -11.0 & -4.5 & -11.0 & -4.9 & -3.4 & -3.8 & -4.1 \\
\hline Current transfers (net) ${ }^{2}$ & 223.8 & 313.1 & 396.8 & 301.8 & 313.9 & 292.1 & 374.1 & 415.0 & 444.5 \\
\hline Private & 31.3 & 35.1 & 48.3 & 52.5 & 62.5 & 57.0 & 70.7 & 76.5 & 82.7 \\
\hline Public $^{3}$ & 192.5 & 278.0 & 348.5 & 249.3 & 251.3 & 235.1 & 303.4 & 338.5 & 361.7 \\
\hline Of which: HIPC grants & 23.0 & 27.4 & 30.6 & 9.3 & 24.8 & 8.6 & 8.6 & 7.6 & 7.1 \\
\hline Current account balance (including official transfers) & -131.1 & -55.5 & -66.5 & -254.3 & -160.8 & -256.4 & -226.1 & -202.1 & -211.4 \\
\hline Current account balance (excluding official transfers) & -323.6 & -333.5 & -414.9 & -503.6 & -412.1 & -491.4 & -529.5 & -540.6 & -573.2 \\
\hline Capital account & 41.1 & 60.6 & 103.2 & 1323.4 & 1323.2 & 103.2 & 106.3 & 152.0 & 175.3 \\
\hline Capital transfers & 41.1 & 60.6 & 103.2 & 173.5 & 173.2 & 103.2 & 106.3 & 152.0 & 175.3 \\
\hline IMF-MDRI relief & & & & 76.6 & 76.6 & & & & \\
\hline Debt forgiveness (IDA/AfDF-MDRI Relief) & & & & 1150.0 & 1150.0 & & & & \\
\hline Financial account & 20.6 & 59.3 & 74.3 & -1085.5 & -1079.9 & 137.1 & 87.5 & -10.9 & 83.4 \\
\hline Direct investment & 4.7 & 3.6 & 10.6 & 25.0 & 14.0 & 13.0 & 26.0 & 19.2 & 20.1 \\
\hline Public sector capital & 7.7 & 72.0 & 48.3 & 48.0 & 44.4 & 116.8 & 60.9 & 62.5 & 61.8 \\
\hline Long-term borrowing ${ }^{3}$ & 39.9 & 109.3 & 83.3 & 59.5 & 68.3 & 127.9 & 70.8 & 71.7 & 70.8 \\
\hline Principal not yet due rescheduled & 6.7 & 0.0 & 0.0 & 0.0 & 0.0 & 0.0 & 0.0 & 0.0 & 0.0 \\
\hline Scheduled amortization ${ }^{4}$ & 32.2 & 37.3 & 35.0 & 11.6 & 23.9 & 11.1 & 9.9 & 9.2 & 9.0 \\
\hline Principal not yet due & 6.7 & 0.0 & 0.0 & 0.0 & 0.0 & 0.0 & 0.0 & 0.0 & 0.0 \\
\hline Other capital $^{5}$ & 8.3 & -16.2 & 15.3 & -1158.5 & -1138.3 & 7.3 & 0.6 & -92.6 & 1.5 \\
\hline IDA/AfDF-relief & & & & -1150.0 & -1150.0 & & & & \\
\hline Capital and financial account balance & 61.8 & 119.9 & 177.7 & 237.9 & 243.3 & 240.2 & 193.8 & 141.1 & 258.7 \\
\hline Errors and omissions & 35.7 & 25.8 & 0.0 & 0.0 & 0.0 & 0.0 & 0.0 & 0.0 & 0.0 \\
\hline Overall balance & -33.6 & 90.2 & 111.2 & -16.1 & 82.5 & -16.2 & -32.3 & -61.0 & 47.3 \\
\hline Financing & 33.6 & -90.2 & -111.3 & 16.1 & -82.5 & 16.2 & 32.9 & 56.7 & -51.6 \\
\hline Change in net foreign assets of NBR (increase -) & 27.1 & -101.4 & -111.3 & 16.1 & -82.5 & 16.2 & 32.9 & 56.7 & -51.6 \\
\hline Net credit from the Fund & -0.9 & -3.6 & -8.2 & -72.5 & -72.5 & 3.3 & 3.3 & 3.3 & 1.7 \\
\hline Disbursements/purchases & 0.8 & 1.7 & 1.7 & 4.1 & 4.1 & 3.3 & 3.3 & 3.3 & 1.7 \\
\hline Repayments/repurchases & -1.7 & -5.3 & -9.8 & -76.6 & -76.6 & 0.0 & 0.0 & 0.0 & 0.0 \\
\hline Change in other gross official reserves (increase -) & 26.0 & -96.9 & -91.3 & 88.5 & -33.8 & -6.6 & 52.2 & 33.1 & -55.4 \\
\hline Change in other foreign liabilities (increase + ) & 1.9 & -0.9 & -11.9 & 0.1 & 23.8 & 19.6 & -22.5 & 20.3 & 2.1 \\
\hline Change in arrears (decrease -) & -22.6 & 1.3 & 0.0 & 0.0 & 0.0 & 0.0 & 0.0 & 0.0 & 0.0 \\
\hline Accumulation & 1.3 & 1.3 & 0.0 & 0.0 & 0.0 & 0.0 & 0.0 & 0.0 & 0.0 \\
\hline Reduction & 23.8 & 0.0 & 0.0 & 0.0 & 0.0 & 0.0 & 0.0 & 0.0 & 0.0 \\
\hline Exceptional financing & 29.1 & 10.0 & 0.0 & 0.0 & 0.0 & 0.0 & 0.0 & 0.0 & 0.0 \\
\hline Cancellation & 0.6 & 0.6 & 0.0 & 0.0 & 0.0 & 0.0 & 0.0 & 0.0 & 0.0 \\
\hline Arrears & 0.0 & 0.0 & 0.0 & 0.0 & 0.0 & 0.0 & 0.0 & 0.0 & 0.0 \\
\hline Current debt service & 0.6 & 0.6 & 0.0 & 0.0 & 0.0 & 0.0 & 0.0 & 0.0 & 0.0 \\
\hline Rescheduling & 28.5 & 9.4 & 0.0 & 0.0 & 0.0 & 0.0 & 0.0 & 0.0 & 0.0 \\
\hline Stock of arrears & 23.1 & 0.0 & 0.0 & 0.0 & 0.0 & 0.0 & 0.0 & 0.0 & 0.0 \\
\hline Arrears & 5.5 & 9.4 & 0.0 & 0.0 & 0.0 & 0.0 & 0.0 & 0.0 & 0.0 \\
\hline Financing need & 0.0 & 0.0 & 0.0 & 0.0 & 0.0 & 0.0 & 1.0 & 4.3 & 4.4 \\
\hline \multicolumn{10}{|l|}{ Memorandum items: } \\
\hline \multicolumn{10}{|l|}{ Current account deficit (Percent of GDP) } \\
\hline Excluding official transfers & -19.2 & -18.2 & -19.4 & -21.4 & -16.7 & -19.2 & -19.3 & -17.7 & -16.9 \\
\hline Including official transfers & -7.8 & -3.0 & -3.1 & -10.8 & -6.5 & -10.0 & -8.2 & -6.6 & -6.2 \\
\hline Gross official reserves & 217.6 & 314.5 & 405.8 & 317.3 & 439.6 & 323.9 & 387.4 & 354.3 & 409.7 \\
\hline Gross official reserves (Months of imports of G\&S) & 5.0 & 5.7 & 6.4 & 4.9 & 6.0 & 4.8 & 5.1 & 4.4 & 4.5 \\
\hline Overall balance (percent of GDP) & -2.0 & 4.9 & 5.2 & -0.7 & 3.4 & -0.6 & -1.2 & -2.0 & 1.4 \\
\hline Total external debt ${ }^{6}$ & 1572.4 & 1685.3 & 1510.0 & 354.0 & 362.7 & 478.4 & 430.1 & 499.0 & 567.6 \\
\hline Total external debt (percent of GDP) & 93.4 & 91.9 & 70.7 & 15.0 & 14.7 & 18.7 & 15.7 & 16.3 & 16.8 \\
\hline Debt service ratio after HIPC and MDRI debt relief ${ }^{7}$ & 11.1 & 9.6 & 7.2 & 5.0 & 2.7 & 5.0 & 1.6 & 1.6 & 1.6 \\
\hline \multicolumn{10}{|c|}{ Sources: Rwandese authorities; and IMF staff estimates and projections } \\
\hline \multicolumn{10}{|l|}{${ }^{1}$ Including interest due to the Fund. } \\
\hline${ }^{2}$ Current transfers include disbursed budgetary and $\mathrm{HI}$ & itarian and & echnical a & sistance. & & & & & & \\
\hline${ }^{3}$ Includes project loans and budgetary loans. & & & & & & & & & \\
\hline${ }^{4}$ Excluding Fund. & & & & & & & & & \\
\hline $\begin{array}{l}{ }^{5} \text { Other capital includes long-term private capital, comn } \\
\text { For } 2004 \text {, the net outflow is caused by the amortizat }\end{array}$ & $\begin{array}{l}\text { the net } f \\
\text { mercial lc }\end{array}$ & $\begin{array}{l}\text { ign ass } \\
\text { contra }\end{array}$ & $\begin{array}{l}\text { of comme } \\
\text { in } 2003 \text { ? }\end{array}$ & $\begin{array}{l}\text { ercial banks, } \\
\text { and linked to }\end{array}$ & $\begin{array}{l}\text { nrecord } \\
\text { al projec }\end{array}$ & ports. & & & \\
\hline
\end{tabular}

(CInternational Monetary Fund. Not for Redistribution 
Table 5. Rwanda: External Financing Requirement and Sources, 2003-09

(In millions of U.S. dollars, unless otherwise indicated)

\begin{tabular}{|c|c|c|c|c|c|c|c|}
\hline & 2003 & 2004 & 2005 & 2006 & 2007 & 2008 & 2009 \\
\hline Resource balance & -324.4 & -334.4 & -438.3 & -496.7 & -616.6 & -641.6 & -690.2 \\
\hline Exports goods and nonfactor services & 139.5 & 189.7 & 227.9 & 264.6 & 280.3 & 304.9 & 322.1 \\
\hline Imports goods and nonfactor services & 463.9 & 524.1 & 666.1 & 761.3 & 896.9 & 946.6 & $1,012.3$ \\
\hline \multirow{2}{*}{$\begin{array}{l}\text { Noninterest current account } \\
\text { (excluding official transfers) }^{1}\end{array}$} & & & & & & & \\
\hline & -312.9 & -319.8 & -403.9 & -447.0 & -560.7 & -566.1 & -596.9 \\
\hline Scheduled interest & -10.7 & -13.7 & -11.0 & -11.0 & -3.4 & -3.7 & -4.1 \\
\hline Capital and financial account ${ }^{2}$ & 9.8 & -24.1 & -8.9 & 1.7 & 26.9 & 32.9 & 44.6 \\
\hline Scheduled amortization & -32.2 & -37.3 & -35.0 & -23.9 & -9.9 & -9.2 & -9.0 \\
\hline Other capital & 42.0 & 13.2 & 26.2 & 25.6 & 36.8 & 42.1 & 53.6 \\
\hline \multicolumn{8}{|l|}{ Of which: IMF-MDRI } \\
\hline Increase in net official reserves (excluding IMF -) & 27.9 & -97.8 & -103.1 & 37.1 & 52.1 & -28.8 & -51.3 \\
\hline \multirow[t]{2}{*}{ IMF repurchases/repayments } & -1.7 & -5.3 & -9.8 & -76.6 & 0.0 & 0.0 & 0.0 \\
\hline & 12.9 & -12.6 & 26.0 & 25.6 & 36.8 & 42.1 & 53.6 \\
\hline Changes in arrears (decrease $=-$ ) & -22.6 & 1.3 & 0.0 & 0.0 & 0.0 & 0.0 & 0.0 \\
\hline External financing requirements & -310.2 & -459.5 & -536.8 & -495.8 & -485.0 & -565.7 & -607.7 \\
\hline Disbursements: Existing & 274.4 & 449.6 & 536.7 & 495.7 & 485.0 & 565.7 & 607.7 \\
\hline Grants (project and nonproject) & 233.6 & 338.6 & 451.7 & 430.9 & 410.4 & 491.2 & 534.2 \\
\hline Project (Public Investment Program) & 41.1 & 60.6 & 103.2 & 174.7 & 107.1 & 152.6 & 172.5 \\
\hline Nonproject $^{3}$ & 192.5 & 278.0 & 348.5 & 256.3 & 303.3 & 338.5 & 361.7 \\
\hline Loans (project and nonproject) & 39.9 & 109.3 & 83.3 & 60.7 & 71.3 & 71.3 & 71.8 \\
\hline Purchases from IMF & 0.8 & 1.7 & 1.7 & 4.1 & 3.3 & 3.3 & 1.7 \\
\hline Exceptional financing (signed) & 29.1 & 10.0 & 0.0 & 0.0 & 0.0 & 0.0 & 0.0 \\
\hline Principal not yet due rescheduled ${ }^{4}$ & 6.7 & 0.0 & 0.0 & 0.0 & 0.0 & 0.0 & 0.0 \\
\hline Available financing & 310.2 & 459.5 & 536.7 & 495.7 & 485.0 & 565.7 & 607.7 \\
\hline Residual financing gap ${ }^{5}$ & 0.0 & 0.0 & 0.0 & 0.0 & 0.0 & 0.0 & 0.0 \\
\hline
\end{tabular}

Sources: Rwandese authorities; and IMF staff estimates and projections.

${ }^{1}$ Excludes budgetary and HIPC grants, and humanitarian and technical assistance.

2 Includes errors and omissions for the past years.

${ }^{3}$ Includes disbursed budgetary grants to the central government (including HIPC grants) and grants in the form of humanitarian and technical assistance.

${ }^{4}$ In 2003, reflects the debt rescheduling agreement with the OPEC Fund.

${ }^{5}$ A negative sign implies a financing gap. 
Table 6. Rwanda: External Public Debt and Debt Service, 2003-09 ${ }^{1}$

(Millions of U.S. dollars, unless otherwise indicated; end of period)

\begin{tabular}{|c|c|c|c|c|c|c|c|c|c|}
\hline & \multirow[t]{2}{*}{2003} & \multirow[t]{2}{*}{2004} & \multirow[t]{2}{*}{2005} & \multicolumn{2}{|c|}{2006} & \multicolumn{2}{|c|}{2007} & \multirow[t]{2}{*}{2008} & \multirow[t]{2}{*}{2009} \\
\hline & & & & $\begin{array}{c}\text { Country } \\
\text { Report } \\
\text { No. 06/245 }\end{array}$ & Proj. & $\begin{array}{c}\text { Country } \\
\text { Report } \\
\text { No. 06/245 }\end{array}$ & Proj. & & \\
\hline Total external debt outstanding ${ }^{2}$ & $1,572.4$ & $1,685.3$ & $1,510.0$ & 354.0 & 354.0 & 478.4 & 421.9 & 490.4 & 559.7 \\
\hline Multilateral & $1,388.6$ & $1,493.1$ & $1,478.1$ & 340.7 & 340.7 & 465.9 & 409.5 & 478.7 & 549.0 \\
\hline Of which: IDA & 912.9 & $1,018.7$ & 983.0 & 186.6 & 186.6 & 314.5 & 258.0 & 331.3 & 403.0 \\
\hline IMF & 91.9 & 92.2 & 77.2 & 5.8 & 5.8 & 9.1 & 9.1 & 12.3 & 14.0 \\
\hline Bilateral $^{3}$ & 183.7 & 189.5 & 31.8 & 13.3 & 13.3 & 12.5 & 12.5 & 11.6 & 10.8 \\
\hline Paris Club & 89.0 & 94.9 & 0.0 & 0.0 & 0.0 & 0.0 & 0.0 & 0.0 & 0.0 \\
\hline Non-Paris Club & 94.8 & 94.5 & 31.8 & 13.3 & 13.3 & 12.5 & 12.5 & 11.6 & 10.8 \\
\hline Debt service due & 44.6 & 56.5 & 56.0 & 61.4 & 67.1 & 69.9 & 69.7 & 69.6 & 67.9 \\
\hline Principal & 33.9 & 42.6 & 45.0 & 48.7 & 53.6 & 55.7 & 56.3 & 56.1 & 54.4 \\
\hline Interest & 10.7 & 13.9 & 11.0 & 12.7 & 13.5 & 14.2 & 13.4 & 13.5 & 13.5 \\
\hline Total debt relief ${ }^{4,5}$ & 29.1 & 38.2 & 44.6 & 9.3 & 59.9 & 8.6 & 65.3 & 64.6 & 62.4 \\
\hline Multilateral & 23.0 & 28.2 & 38.1 & 9.3 & 56.8 & 8.6 & 59.9 & 59.7 & 58.1 \\
\hline Bilateral $^{5}$ & 6.1 & 10.0 & 6.5 & 0.0 & 3.0 & 0.0 & 5.3 & 4.9 & 4.3 \\
\hline Debt service after HIPC and MDRI & 15.5 & 18.3 & 16.8 & 3.9 & 7.3 & 4.9 & 4.5 & 5.0 & 5.5 \\
\hline Principal & 12.3 & 10.6 & 11.0 & 3.9 & 4.9 & 4.5 & 1.6 & 1.6 & 1.5 \\
\hline Interest & 3.3 & 7.7 & 5.8 & 2.7 & 2.4 & 2.8 & 2.9 & 3.4 & 4.0 \\
\hline \multicolumn{10}{|l|}{ Memorandum items: } \\
\hline Total debt (Percent of GDP) & 93.4 & 91.9 & 70.7 & 15.0 & 14.8 & 18.7 & 15.7 & 16.5 & 17.1 \\
\hline \multicolumn{10}{|l|}{ Debt service due } \\
\hline Percent of exports of goods and services & 32.0 & 29.8 & 24.6 & 24.7 & 26.7 & 25.7 & 25.4 & 23.3 & 21.7 \\
\hline Percent of government revenue ${ }^{6}$ & 19.6 & 22.1 & 17.4 & 18.5 & 18.6 & 19.1 & 17.3 & 15.4 & 13.4 \\
\hline Percent of government current expenditure and net lenc & 11.1 & 11.8 & 9.2 & 9.3 & 9.8 & 10.0 & 8.8 & 7.9 & 7.0 \\
\hline \multicolumn{10}{|l|}{ Debt service after HIPC and MDRI Initiative } \\
\hline Percent of exports of goods and services ${ }^{5}$ & 11.1 & 9.7 & 7.2 & 5.0 & 2.7 & 5.0 & 1.6 & 1.6 & 1.7 \\
\hline Percent of government revenue ${ }^{6}$ & 6.8 & 7.2 & 5.2 & 1.2 & 2.0 & 1.3 & 1.1 & 1.1 & 1.1 \\
\hline Percent of government current expenditure and net lenc & 3.9 & 3.8 & 2.8 & 0.6 & 1.1 & 0.7 & 0.6 & 0.6 & 0.6 \\
\hline Net present value (NPV) of debt (US\$ million) ${ }^{7}$ & 398.4 & 452.3 & 283.1 & 162.7 & 162.8 & 225.3 & 200.4 & 239.8 & 279.5 \\
\hline NPV of debt-to-exports ratio ${ }^{7}$ & 285.6 & 239.4 & 149.8 & 65.6 & 61.5 & 82.9 & 73.7 & 81.1 & 89.6 \\
\hline
\end{tabular}

Sources: Rwandese authorities; and IMF staff estimates and projections.

${ }^{1}$ Based on WEO exchange rate projections for 2006-09.

${ }^{2}$ After rescheduling, including arrears and new debt (the latter includes assumed project and budgetary disbursements for the period 2006-09).

${ }^{3}$ All Paris Club debt is precut off-date debt. Assumes full delivery of assistance under the Enhanced HIPC Initiative by all creditors.

${ }^{4}$ Reflects traditional debt relief from bilateral creditors and MDRI and enhanced HIPC Initiative assistance for both multilateral and bilateral creditors. Also includes additional bilateral debt relief delivered at the completion point.

${ }^{5}$ Projections for 2006 and 2007 reflect corrections made to spreadsheet errors presented in Country Report 06/245.

${ }^{\circ}$ Excluding grants.

'Based on completion point LIC DSA in 2003-04; 2005 onward is based on current DSA with topping up in 2005 and MDRI in 2006. 
Table 7. Rwanda: Structural Conditionality, 2006-07

\begin{abstract}
Public expenditure management
Issue draft accounting instructions, forms, and procedures for budget users.

Issue guidelines on doing bank reconciliations and accounting for line ministries, provinces,

autonomous agencies, and extrabudgetary funds.

Issuing the first report of a monitoring system of project accounts. The report will show (1) the inand outflows (the latter broken down into foreign exchange and domestic spending) on a quarterly basis of project accounts at the NBR listed in paragraph 42 of the TMU for January-March 2006 and April-June 2006; and (2) rolling spending plans for the accounts on a quarterly basis, broken down into foreign exchange and domestic spending for July 2006-June 2007.
\end{abstract}

\section{Governance}

Reconcile the databases maintained by the Ministry of Finance and the National Bank of Rwanda of the debt service payments as of end-June 2006.

Cabinet to approve draft laws on establishing a commercial registration agency and on intellectual property.

\section{Financial sector}

Submit to Parliament amendments to the banking law to bring the legal framework for banking supervision more in line with international best practice (Basel Core Principles). The amendments to the banking law shall, as a minimum, and consistent with the Aide-Memoire entitled "Revision of Legal Framework for Banking Supervision Amendments to Central Bank and Banking Law" dated December 2005: (i) provide limits on large exposures and connected lending, i.e., by eliminating carve-outs for mortgage collateral, and prudential safeguards against connected lending; (ii) grant NBR power to approve and refuse the acquisitions of significant ownership holdings and powers to enforce refusal decisions in cases of noncompliance; (iii) provide NBR with powers to regulate banks' corporate governance; (iv) provide NBR with a set of specific corrective measures against unsound banking practices; and (v) provide a framework for NBR to impose reorganization and liquidation measures on banks, whereby the role of the special commissioner is strengthened.

\section{Civil service reform}

Undertake a comprehensive review of the wage structure of the public sector, including fringe benefits and wages included in transfers to local governments.

\section{Agricultural sector}

Adopt and publish a national fertilizer distribution strategy.
Status
December 31, 2006

October 31, 2006

August 31, 2006

September 30, 2006

October 31, 2006

September 30, 2006

December 31, 2006 Criterion

\author{
Performance Not met. \\ Criterion \\ Implemented as \\ prior action
}

Benchmark

Benchmark

Met.
Performance

Benchmark Met.

Benchmark Met.

Met.
February 28, $2007 \quad$ Benchmark 
Table 8. Rwanda Financial Soundness Indicators for the Banking Sector, 2000-05

(In percent, at year's end, unless otherwise indicated)

\begin{tabular}{|c|c|c|c|c|c|c|}
\hline & 2000 & 2001 & 2002 & 2003 & 2004 & 2005 \\
\hline \multicolumn{7}{|l|}{ Capital Adequacy } \\
\hline Regulatory capital to risk-weighted assets & 14.2 & 16.6 & 12.5 & 14.6 & 18.3 & 14.7 \\
\hline Percentage of banks greater or equal to 10 percent & 81.8 & 72.7 & 72.7 & 63.6 & 100.0 & 83.3 \\
\hline Percentage of banks below 10 and above 6 percent minimum & 0.0 & 9.1 & 0.0 & 18.2 & 0.0 & 0.0 \\
\hline Percentage of banks below 6 percent minimum & 18.2 & 18.2 & 27.3 & 18.2 & 0.0 & 16.7 \\
\hline Capital (net worth) to assets & 18.1 & 20.0 & 15.1 & 18.0 & 11.7 & 9.1 \\
\hline \multicolumn{7}{|l|}{ Asset quality } \\
\hline Foreign exchange loans to total loans & 0.1 & 0.0 & 0.1 & 0.0 & 0.3 & 0.0 \\
\hline \multicolumn{7}{|l|}{ Past-due loans to gross loans } \\
\hline Nonperforming loans to total loans & 53.1 & 74.1 & 57.0 & 52.0 & 27.0 & 27.2 \\
\hline Watch-listed loans & n.a & n.a & n.a & n.a & n.a & 13.3 \\
\hline Provision as percent of past-due loans & n.a & n.a & n.a & n.a & n.a & 56.7 \\
\hline \multicolumn{7}{|l|}{ Earnings and profitability } \\
\hline \multicolumn{7}{|l|}{ Net profit (before tax)/net income } \\
\hline Return on assets & 1.5 & 0.4 & -5.0 & 1.4 & 2.2 & 1.5 \\
\hline Return on equity & 23.9 & 5.1 & -125.3 & 31.1 & 21.6 & 16.5 \\
\hline Expense/income & 72.8 & 90.1 & 141.1 & 75.5 & 73.1 & 71.0 \\
\hline \multicolumn{7}{|l|}{ Interest rate spread (deposit money banks) ${ }^{1}$} \\
\hline Lending rates minus demand deposit rates & n.a & n.a & n.a & n.a & n.a & n.a \\
\hline Lending rates minus saving deposit rates & n.a & n.a & 7.6 & 7.6 & 7.1 & 8.1 \\
\hline \multicolumn{7}{|l|}{ Liquidity } \\
\hline Liquid assets/total assets & 6.1 & 6.0 & 9.4 & 11.1 & 16.2 & 35.0 \\
\hline Liquid assets/short term liabilities & n.a & n.a & n.a & n.a & n.a & 59.0 \\
\hline Loan/deposits & n.a & n.a & n.a & n.a & n.a & 74.0 \\
\hline Foreign exchange liabilities/total liabilities & 30.3 & 23.9 & 23.4 & 27.5 & 24.3 & 27.5 \\
\hline \multicolumn{7}{|l|}{ Sensitivity to market risk } \\
\hline Net foreign exchange assets (liabilities) to shareholders' funds & -5.3 & 3.6 & 2.3 & -4.5 & 6.1 & 7.0 \\
\hline
\end{tabular}

Sources: National Bank of Rwanda and staff calculations.

${ }^{1}$ There is a predominance of noninterest bearing demand deposits. 


\title{
APPENDIX I-RWANDA: LETTER OF INTENT
}

Kigali, January 10, 2007

\author{
Mr. Rodrigo de Rato y Figaredo \\ Managing Director \\ International Monetary Fund \\ Washington, D.C. 20431 \\ U.S.A.
}

Dear Mr. de Rato:

1. The Government of Rwanda is implementing a financial and economic program supported under the Fund's Poverty Reduction and Growth Facility (PRGF), which was approved by the Executive Board on June 5, 2006. We hereby transmit the Memorandum of Economic and Financial Policies (MEFP), which reviews recent economic developments and progress in the implementation of our program in the first half of 2006; and sets out policies the Government intends to pursue in the remainder of 2006 and 2007.

2. Program implementation under the PRGF arrangement has been broadly on track. All quantitative performance criteria and indicative targets for end-June 2006 were met with the exception of the performance criterion (PC) on priority spending. The latter was affected by technical difficulties in the Lake Kivu methane gas project for electricity generation, which resulted in a spending delay. With these difficulties now resolved, about half of the spending was effected in September and the other half will be effected in December. With an acceleration in other priority spending, the end-September target on priority spending was met and we also expect to meet the end-December target. On the structural front, the endSeptember 2006 PC on the submission to parliament of amendments to the banking law was met. However, issuing the first quarterly report of a monitoring system of project accounts, an end-August 2006 PC, was delayed. It was implemented on January 9 as a prior action. As the nonobservance of both performance criteria was of a temporary nature, we are requesting waivers for the nonobservance of the quantitative PC on priority spending and the structural $\mathrm{PC}$ on the issuance of the report on the monitoring of project accounts.

3. In support of our policies described in the MEFP, the Government of Rwanda requests the completion of the first review under the PRGF arrangement and the disbursement of the second loan of SDR 1.14 million.

4. The Government of Rwanda believes that the policies set forth in the attached MEFP are adequate to achieve the objectives of its program, but it will take any further measures that may become appropriate for this purpose. The Government of Rwanda will consult with 
the Fund on the adoption of these measures and in advance of revisions to the policies contained in the MEFP, in accordance with the Fund's policies on such consultations.

5. The Government of Rwanda authorizes the publication and distribution of this letter and MEFP together with the related staff report.

Sincerely yours,

/s /

François Kanimba

Governor

National Bank of Rwanda
/ s /

James Musoni

Minister of Finance and

Economic Planning

Attachments: Memorandum of Economic and Financial Policies

Technical Memorandum of Understanding 


\section{APPENDIX I-ATTACHMENT I \\ Rwanda: Memorandum of Economics And Financial Policies}

(January 10, 2007)

\section{Rwanda's medium-term economic program is supported by an arrangement} under the Poverty Reduction and Growth Facility over 2006-2009. We are committed to reducing poverty through preserving macroeconomic stability, boosting economic growth, and implementing policies targeted at improving the livelihood of the poor. This memorandum is consistent with the goals established in the Poverty Reduction and Strategy Paper and three annual progress reports. The memorandum reviews the recent performance under the program and describes the policies and targets for the remainder of 2006 and 2007.

\section{RECENT ECONOMIC DEVELOPMENTS}

2. Growth remains strong, but inflation has been rising. GDP growth is expected to exceed 4 percent in 2006, owing to buoyant activity in manufacturing as well as the services and financial sectors, which are offsetting a decline in agricultural production. 12-month endof-period inflation has increased to 11 percent in November, owing mostly to increased food and energy prices, but annual average inflation in November was at 8.3 percent.

3. The fiscal program has remained broadly on track through September 2006.

- $\quad$ Fiscal policy in the first half of 2006 was tighter than programmed because of strong revenue. Revenue over performed by about $3 / 4$ percent of GDP, reflecting mostly an increase in payroll-related taxes due to a widening of the tax net and a broadening of the tax base to fringe benefits. With overall spending broadly in line with the program, the end-June targets on the domestic deficit was met by a wide margin. ${ }^{1}$ However, technical delays in the Lake Kivu project led a postponement of the spending, and thus end-June priority spending was below its target by 0.1 percent of GDP. $^{2}$

- $\quad$ Preliminary data for September point to continuing revenue buoyancy. Thus, despite some unforeseen outlays, the September target on the domestic deficit was met (as were the ones on priority spending and arrears clearance). The end-September target on the net credit to government was met by a small margin. However, a discrepancy of about $\mathrm{Rf} 10$ billion with revenue and expenditure data indicate that this reflected that expenditure released to budgetary agencies was not yet effected.

\footnotetext{
${ }^{1}$ Also the end-June target on domestic arrears clearance was met.

${ }^{2}$ Without this delay, the end-June target would have been met.
} 


\section{While the reserve money targets for end-June and end-September 2006 were met, monetary management was rendered difficult because of:}

- $\quad$ A larger-than-programmed domestic spending component of the budget. The National Bank of Rwanda (NBR) could not step up the sales of foreign exchange sufficiently to keep reserve money within the program limits and thus had to resort to sterilization using domestic instruments. As a result, consolidated domestic debt stayed broadly at its end-2005 level (instead of being reduced as envisaged under the program) and net international reserves exceeded both the end-June and endSeptember targets.

- $\quad$ A large increase in broad money, owing mostly to an increase in private sector credit. ${ }^{3}$ Credit increased by 26 percent (year-on year) in September, pushing broad money well beyond the indicative target (by 8 percent).

5. The external current account is expected to be tighter than programmed. This reflects mostly lower-than-programmed government imports during January-September, and the NBR's increased sterilization of government spending through domestic instruments. Exports are projected to slightly exceed projections, due to higher volumes in coffee and tea. The nominal exchange rate depreciated by 1.5 percent during January-September, while there was a 5 percent appreciation in the real effective exchange rate .

\section{Substantial progress has been made on the structural front.}

- $\quad$ To bring banking supervision in line with international best practice, amendments to the banking law were submitted to Parliament in September (performance criterion). Moreover, in June 2006, the NBR closed 9 mismanaged Microfinance Institutions (MFIs), which had experienced significant deposit withdrawals. To help stabilize the situation and avoid contagion, we reimbursed deposit holders for half of their net deposits (about 0.1 percent of GDP) and appointed the Union des Banques Populaires (UBPR) to administer the recovery of outstanding loans.

- $\quad$ To enhance public financial management, guidelines for undertaking bank reconciliations and accounting for government agencies were issued in mid-2006 (end-October benchmark). To improve the monitoring of project accounts (endAugust performance criterion), the backward-looking part on historical quarterly disbursements and expenditures was completed on time, while the forward-looking part on quarterly projections for January-December 2007 was implemented in

\footnotetext{
${ }^{3}$ Broad money was also affected by a temporary large deposit of a repatriated private investment.
} 
January (prior action). The Organic Budget Law (OBL) was gazetted in September and its supporting financial regulations were approved by Cabinet in October.

- To facilitate decentralization, Cabinet approved the creation of a Decentralization Unit in the Ministry of Finance in October.

- $\quad$ To strengthen debt management, we have reconciled in September the debt databases maintained by the Ministry of Finance and the NBR as at end-June 2006 (benchmark). The two institutions now maintain a unified database.

- $\quad$ To improve the investment climate, Cabinet approved the draft laws on establishing a commercial registration agency and on intellectual property in June (benchmark) and the laws are now at parliament.

- $\quad$ As a long-term solution to the management of Prime Holdings' two hotels, a 30-year lease contract has been signed with an international hotel chain.

\section{MEDiUm-Term STRATEGY}

7. As outlined in our MEFP of May 2006, our medium-term strategy is based on the following pillars, which will also be reflected in the new PRSP (expected to be finalized in the first half of 2007):

- $\quad$ Removing obstacles to private sector development to make it the engine of growth. To this end, we will continue building human capital through better health and education and address Rwanda's poor infrastructure links, insufficient and expensive energy supply, its shallow financial system, and high costs of doing business.

- $\quad$ Enhancing agricultural development and trade as vehicles to empower the poor by having them participate in economic activity.

- Improving the delivery of public services through better expenditure management, civil service reform, and better analyses of the links between policies and growth and poverty reduction.

- $\quad$ Relying on sustainable financing sources with an emphasis on grant financing and raising the revenue ratio in the long run.

While our medium-term macroeconomic framework is based on prudent projections for growth and external assistance, we believe that that steadfast implementation of our reform agenda is likely to lead to substantially higher-than-projected growth and we also hope that it could facilitate higher levels of external assistance. Moreover, to further improve the policy dialogue with stakeholders and development partners, starting with the new PRSP, we will maintain a rolling medium-term expenditure and macroeconomic framework. 


\section{Policies for the Remainder OF 2006}

\section{Despite unforeseen outlays, we expect to meet most end-2006 fiscal targets.}

Revenue over performance is expected to more than cover unanticipated spending for restocking the fuel reserve, a transfer to the deposit holders of the recently closed MFIs, and a higher-than-anticipated domestic interest bill. However, if AU reimbursements of our peacekeeping efforts continue to be delayed (see paragraph 15), it is possible that we will not meet the end-2006 target on net credit to government.

\section{To prevent the recent credit expansion from fueling inflation, the NBR will} maintain a tight monetary policy. It will continue to monitor inflation and private sector credit and stand ready to tighten the stance, if needed. Moreover, the NBR audited a bank, which had shown a particularly large increase in the consumer credits. As this audit found that the credits had not been provided with sufficient guarantees, the bank has now slowed down the pace of granting new credits.

\section{The Program For 2007}

\section{Our policies in $\mathbf{2 0 0 7}$ will continue to safeguard macroeconomic stability while} laying the base for stronger medium-term growth. They are based on a real growth rate of $4 \frac{1}{2}-6 \frac{1}{2}$ percent, keeping inflation at 5 percent, and a level of international reserves of at least 4.3 months of imports. Macroeconomic and structural policies will focus on improving the living standards of the poor, and growth-enhancing investments and reforms.

\section{A. Macroeconomic Program}

\section{Managing the domestic demand impact of fiscal policies will lie at the heart of} our macroeconomic policies. The domestic deficit (excluding spending on demobilization and peace keeping) is projected to increase by about 1 percent of GDP compared with 2006, financed by higher aid flows, but also some draw down of the NBR's reserves. Thus, pressures for a real exchange rate appreciation are expected to continue and could be exacerbated by a likely drawdown of project accounts. ${ }^{4}$ To protect the monetary program and ensure that fiscal policy is not crowding out investment, we will, if grants exceed program projections, release any further spending (so called "contingent spending," see paragraph 14) only in the context of the second review under the PRGF of macroeconomic developments.

\footnotetext{
${ }^{4}$ The programmed draw down of project deposits in 2006 (which stood at 5 percent of GDP at end-2005) did not materialize until September 2006. We are now projecting a partial draw down during 2007.
} 


\section{Fiscal Policy}

\section{Fiscal policy is guided by the following main considerations: ${ }^{5}$}

- $\quad$ On the revenue front, we will maintain the revenue-to-GDP ratio at its 2005 level of 15 percent of GDP, despite a revenue loss of almost $1 / 2$ percent of GDP from the removal of the Magerwa warehouse handling fees and a reduction in the corporate tax rate. Our revenue measures are described in paragraph 13.

- $\quad$ The focus on expenditure lies in increasing the share of priority spending in the budget while improving the quality of public services. Given uncertainties related to weather conditions, the program continues to include an adjuster allowing an increase in the deficit and a commensurate reduction in net foreign assets for food imports related to a food emergency.

\section{Budget}

\section{With respect to revenue measures, we have:}

- Implemented a tax on mobile and fixed telephone usage (revenue gain of 0.1 percent of GDP);

- Increased the excise tax on cigarettes (revenue gain of about 0.1 percent of GDP);

- Introduced licenses for importers (replacing part of the Magerwa loss; revenue gain of about 0.1 percent of GDP);

- Increased the reference price on petroleum (revenue gain of about 0.1 percent of GDP).

These measures are expected to offset the revenue loss. In the case of a shortfall, we will increase the reference prices for petroleum products. Moreover, on the basis of a survey on the informal sector, we will study ways to increase our tax base in the medium term.

\section{The budget focuses on improving the living standards of our population while} boosting the economy's productivity. Spending will increase by about 2 percent of GDP and could increase further, including through the release of contingent spending of about 1 percent of GDP (half of which is for priorities) and spending for education through the Fast

\footnotetext{
${ }^{5}$ While the budget submitted to Parliament was not fully in line with program understandings, amendments were made during the budget discussions and the published budget is in line with the program.
} 
Track Initiative of about 1 percent of GDP. ${ }^{6}$ In releasing contingent spending, we will ensure that more than half is going to priorities (in particular rural terracing, the common development fund, energy savings devices, forestation and road maintenance).

Fiscal Program, 2006-07

(Percent of GDP)

\begin{tabular}{|c|c|c|c|}
\hline & 2006 & $\begin{array}{c}2007 \\
\text { without conting. }\end{array}$ & $\begin{array}{r}2007 \\
\text { with conting. } \\
\end{array}$ \\
\hline Revenue & 15.0 & 15.0 & 15.0 \\
\hline Expenditure (excl. foreign financed projects and interest) & 21.9 & 22.9 & 24.0 \\
\hline Priority & 10.9 & 12.5 & 13.0 \\
\hline Nonpriority & 8.9 & 8.8 & 9.4 \\
\hline Domestic interest & 0.8 & 0.6 & 0.6 \\
\hline Peacekeeping operations & 0.8 & 0.7 & 0.7 \\
\hline Demobilization & 0.5 & 0.3 & 0.3 \\
\hline Domestic fiscal deficit (excl. AU peacekeeping and demobilization) & -5.6 & -6.8 & -7.9 \\
\hline
\end{tabular}

- $\quad$ Priority spending will increase for both social sectors and enhancing the productivity of the economy. An increase in the capitation transfers (i.e., funds provided per pupil) by 60 percent will allow to improve the quality of teaching (see paragraph 15). Moreover, the allocation to the water sector has increased substantially to provide access to safe water to a larger part of the population. Allocations have also been made to establish land offices in districts, which will start the registration of land ownership (the titles could be used as collateral for bank lending). To address the issue of limited and expensive electricity supply, we will continue financing the Lake Kivu project, and start several hydropower electricity projects. The budget also covers Electrogaz's costs related to the rental of diesel generators and the fuel cost of one of the diesel generators (of 5MW) to avoid a further increase in electricity tariffs, which would adversely affect the private sector (see paragraph 15). These subsidies will be for 2007 only as we expect a heavy fuel generator to become operational by end-2007, which would replace some of the more expensive diesel generators. Part of the increase in priority spending (about 0.5 percent of GDP) is financed through the

\footnotetext{
${ }^{6}$ As long as additional grants are available, the program would allow an increase in spending beyond contingent spending and the education spending if it is used for priorities.
} 
MDRI debt relief, which will be used for improving the rural water supply, and spending related to the Lake Kivu project.

Priority spending

(Percent of GDP)

\begin{tabular}{lrrr}
\hline & $\mathbf{2 0 0 6}$ & $\mathbf{2 0 0 7}$ & $\mathbf{2 0 0 7}$ \\
& w/o cont. & with cont. \\
\hline Priority & & $\mathbf{1 0 . 9}$ & $\mathbf{1 3 . 0}$ \\
Agriculture, land, and water & 0.7 & 1.4 & 1.5 \\
Health & 1.4 & 1.6 & 1.6 \\
Education & 4.5 & 4.8 & 4.8 \\
Infrastructure and energy & 1.5 & 2.0 & 2.3 \\
Export and investment promotion & 0.5 & 0.3 & 0.4 \\
Internal security & 0.7 & 0.7 & 0.7 \\
Decentralization & 1.0 & 1.3 & 1.3 \\
Other & 0.6 & 0.5 & 0.5 \\
& & & \\
Memorandum items: & & & $\mathbf{0 . 5}$ \\
Use of MDRI relief & & $\mathbf{0 . 5}$ & $\mathbf{0 . 5}$ \\
Water & $\mathbf{0 . 5}$ & 0.2 & 0.2 \\
Lake Kivu & 0.0 & 0.3 & 0.3 \\
Food imports & 0.3 & 0.0 \\
& & & 0.0 \\
\hline
\end{tabular}

- $\quad$ Nonpriority spending would fall slightly as a percent of GDP (and increase by about $1 / 2$ percent of GDP including contingent spending). This includes about 0.4 percent of GDP for social cohesion (gacaca courts, genocide survivors, prison feeding, repatriation of refugees), 0.6 percent of GDP for administrative issues (including 2 new important embassies, an increase in the transfer to the Rwanda Revenue Authority, new identity cards, the establishment of the accountant general's office and arbitration courts, and the rehabilitation of laboratories for quality control), and 0.3 percent of GDP for ICT, feasibility studies, and new energy savings devices. There also is a substantial decline in some one-off items compared with 2006. 
Nonpriority Spending

(Percent of GDP)

\begin{tabular}{lccc}
\hline & $\mathbf{2 0 0 6}$ & $\mathbf{2 0 0 7}$ & $\mathbf{2 0 0 7}$ \\
& & & \\
without cont. & with cont. \\
\hline Nonpriority & $\mathbf{8 . 9}$ & $\mathbf{8 . 8}$ & $\mathbf{9 . 4}$ \\
& & & 0.3 \\
Gacaca courts & 0.1 & 0.3 & 0.8 \\
Fund for genocide survivors & 0.7 & 0.8 & 0.1 \\
Refugee repatriation & 0.0 & 0.1 & 0.4 \\
Prison feeding and rehabilitation & 0.3 & 0.3 & 0.5 \\
New embassies in Sudan and the Netherlands & 0.4 & 0.5 & 0.1 \\
Identity card & 0.0 & 0.1 & 0.1 \\
Establishment of the Accountant General's Office & 0.0 & 0.1 & 0.1 \\
Establishment of arbitration courts & 0.0 & 0.1 & 0.5 \\
Rwanda Revenue Authority & 0.4 & 0.5 & 0.6 \\
Rehabilitation of special laboratories for quality control & 0.4 & 0.6 & 0.05 \\
Full-year salary impact from new hirees & 0.00 & 0.05 & 0.03 \\
Energy savings devices & 0.00 & 0.01 & 0.1 \\
New ICT projects & 0.0 & 0.0 & 0.2 \\
Feasibility studies for roads, electricity lines & 0.0 & 0.0 & 0.2 \\
Elections & 0.3 & 0.2 & 2.4 \\
Military spending & 2.5 & 2.4 & 0.0 \\
Reimbursing deposit holders of bankrupt MFls & 0.1 & 0.0 & 0.0 \\
Restocking of fuel & 0.2 & 0.0 & 0.0 \\
Transfer to central bank for losses in 2005 & 0.3 & 0.0 & 3.2 \\
Other & 3.2 & 2.9 & \\
& & &
\end{tabular}

\section{On specific spending items:}

- $\quad$ AU peace keeping in Darfur. The AU had agreed to reimburse Rf 9.4 billion in 2006 for outlays related to peace keeping activities, but has, so far, provided only a partial reimbursement of $\mathrm{Rf} 3.1$ billion. While we would be willing to continue our efforts, we are concerned that the ensuing financial burden would hamper our own development and will thus review the situation by January 2007. To monitor the costs associated with the peace keeping, the Auditor General will conduct an audit of the spending in 2006 expenditures that will be published by end-March 2007 . 
- $\quad$ Military spending. Military spending is about 2.4 percent of GDP due to an increase in wages for conscripts, implemented in 2006. This restores their salaries in real terms as of 2000. Our total military outlays are as indicated in the table below.

- $\quad$ Stipends for tertiary education students. To reallocate funds from tertiary to lower (Billions of Rwandese francs)

Military spending

\begin{tabular}{|c|c|c|c|}
\hline & \multicolumn{2}{|c|}{2006} & \multirow[t]{2}{*}{2007} \\
\hline & Program & Rev. Proj. & \\
\hline Wages & 17.9 & 21.0 & 21.0 \\
\hline Current spending & 9.4 & 9.4 & 10.7 \\
\hline Transfers & 1.3 & 1.3 & 1.3 \\
\hline Capital & 0.8 & 0.8 & 1.2 \\
\hline Total & 29.4 & 32.5 & 34.2 \\
\hline Total as percent of GDP & 2.23 & 2.47 & 2.37 \\
\hline
\end{tabular}
education, we are designing a plan to target stipends for tertiary students with a view to gradually reduce the share of students with scholarships to half by 2010 . The implementation plan will be completed by end-June 2007 .

- Capitation grant for primary education. The increase in the capitation grant will allow districts to improve the quality of education by hiring more teachers and providing districts and schools with more control of incentive schemes to reduce teacher absenteeism. With respect to the latter, the ministry of education is designing performance guidelines and contracts, which would be signed between districts and schools. The capitation grants will stay at Rf 2,500 per pupil with the balance of Rf1,500 to be released after contract signature.

- $\quad$ Electricity tariff. We have adopted a structured tariff in October, which will be implemented starting in January 2007. We will review by end-June 2007 the subsidies to Electrogaz. ${ }^{7}$ If they are insufficient to ensure Electrogaz' cost recovery in 2007, we stand ready to raise electricity tariffs. If the needed subsidy is smaller, the savings will be allocated to the agricultural sector.

\section{Fiscal structural reforms}

16. Civil service reform is proceeding. Parliament is reviewing a civil service law, which regulates issues such as recruitment, grading, and promotion and we are also in the process of developing performance standards for ministries and agencies. However, the

\footnotetext{
${ }^{7}$ The size of the subsidy depends on the oil price and when the heavy fuel oil generator will become operational.
} 
review of the wage of the entire civil service, which was to be completed by end-December 2006 (benchmark) is now expected to be delayed to March 2007.

\section{To further enhance the monitoring of project accounts at the NBR, we will} complete a study and publish it on the Ministry of Finance's website by end-September 2007 (1) showing annual budgets with donor disbursements and spending for 2004-2006 as well as quarterly projections (including a breakdown into imports and the domestic component) for July 2007-June 2008; and (2) providing an analysis of the causes for the large accumulation of the specific project accounts since January 1, 2004 (performance criterion).

\section{Public Financial Management (PFM) reforms have been given further impetus} with the establishment of the PFM Steering Committee. A PFM Secretariat at the Ministry of Finance is charged with coordinating, monitoring and evaluating reforms in cooperation with our development partners, who are supporting us through a multi-donor trust fund within the public sector capacity project. Pending the completion of a comprehensive medium-term PFM agenda, expected to be finalized by June 2007, we have developed an interim action matrix including:

- Legal Framework. We plan to conduct by end-2006 workshops for senior and midlevel officials on the implementation of the OBL and its financial regulations. To monitor and review implementation, a progress report on the OBL implementation will be published in September 2007 (benchmark).

- Reconciliation. Agencies have improved the reconciliation of their accounts after the issuance of guidelines in mid-year, but some are still not compliant in submitting their monthly statements. To improve reporting discipline, we wrote in October letters reminding agencies of reporting requirements and indicating that their funds could be cut withheld if they do not comply with the reporting requirements. Acknowledging that further training is needed for budget managers to fully reconcile all bank accounts, line ministries, provinces, and autonomous agencies will be expected to produce the following monthly financial reports: (i) bank reconciliation statement; (ii) budget execution reports, and (iii) statements of revenue and expenditure. The first progress report on financial reporting by budget agencies covering January to March 2007 will be finalized by mid-June 2007 (benchmark).

- $\quad$ Public Accounts. We will produce as envisaged by March 2007 the consolidated government financial statements for 2006. Moreover, to facilitate moving to a treasury single account, we will close by end-June 2007 all dormant government accounts in commercial banks.

- $\quad$ Budget Preparation and Execution. In January 2007 we will begin work on revising the economic and functional classification of the budget for implementation by September 2008. We will also by March 2007, decentralize the authority to 
ministries and districts to effect expenditure commitments and virements. Moreover, line ministries, provinces, and autonomous agencies with extrabudgetary resources will start producing quarterly reports on extrabudgetary resources received.

- Development Planning. In collaboration with donors work has begun on strengthening the performance-based budget. We will by end-December 2007 improve the capacity to undertake strategic planning, logical framework analysis and costing activities. Additionally, by end-September 2007 we plan to produce a poverty profile of provinces, districts, and communities of Rwanda using the results of the census and the household living conditions survey (benchmark).

- Public Procurement. If the Public Procurement Code and the law establishing the Rwanda Public Procurement Regulatory Authority (RPPA) are adopted by end-2006, the RPPA could be operational by March 2007 and, through recruitment and training, procurement capacities could be established in budget agencies by end-2007 with assistance from the World Bank.

\section{Monetary and Exchange Rate Policy}

19. The NBR will continue to use reserve money as the operational target to control inflation. To maintain inflation at 5 percent, it will limit year-end reserve money growth to 13 percent, which would create room for an expansion of credit to the private sector. The NBR will continue to follow closely inflationary developments and aggressively mop up excess liquidity, in particular through sales of foreign exchange.

20. We will allow more flexibility in the exchange rate. Acknowledging that an increase in spending from higher aid flows in 2007 is likely to result in further pressures for a real exchange rate appreciation, we will forcefully implement our intervention strategy. Our interventions will accommodate upward pressures on the exchange rate through a nominal appreciation by raising foreign exchange sales while smoothing short-term market fluctuations. This will ensure that the execution of the 2007 budget does not rekindle inflation or crowd out private investment.

\section{While NBR's foreign exchange auction system serves Rwanda well, we have} started to prepare for a smooth transition to an interbank-based market. The NBR has issued in June an instruction, which (1) improves auction procedures; and (2) abolishes Article 10, which allowed the NBR to check whether the foreign exchange purchased at the auction was used in accordance with regulations. We have also issued amendments to the foreign exchange regulation that address further regulatory constraints and reduce reporting requirements. 


\section{External Sector}

22. Owing to the increase in fiscal spending, we expect the external current account deficit (excluding official transfers) to widen to more than 21 percent of GDP (by almost 2 percent of GDP compared with 2006). Export receipts are projected to grow by at least 7 percent, driven mainly by increased volumes of traditional exports owing to our sectoral strategies (see paragraph 27). The expected increase in imports by more than 20 percent stems from an expansion in the fiscal deficit as well as a draw down of project accounts and the Lake Kivu project.

\section{The implementation of debt relief under the enhanced HIPC Initiative is at an} advanced stage. In addition to the IMF, IDA, and AfDB, completion point and topping up assistance have been provided by BADEA and the OPEC Fund. IFAD, the Kuwait Fund, the Saudi Fund and the EU have provided HIPC debt relief without topping up. Bilateral agreements have now been signed with all Paris Club creditors. Regarding non-Paris Club creditors, China has indicated a willingness to cancel all outstanding loans, while Saudi Arabia and Kuwait noted the possibility of future debt relief. Debt owned to Libya and the Abu Dhabi Fund continues to be passive. ${ }^{8}$

\section{Debt management will be strengthened through improved recording and}

monitoring. A new debt recording system is expected to be in place by January 2007, with a live link between the Treasury and the NBR. Moreover, we have introduced an indicative target on the Net Present Value (NPV) of external debt for 2007, which will ensure that the level of contracted debt remains sustainable and allow a review of the medium-term debt profile (see technical memorandum of understanding, paragraphs 42-44).

\section{At end-November, Rwanda became a member of the East African Community} (EAC) and signed the protocol for a customs union. ${ }^{9}$ We expect that membership will have a positive welfare effect by reducing import prices and also provide an opportunity to promote regional development and address common issues, including infrastructure needs. We will negotiate the terms of the treaty and customs union protocol during 2007 with implementation envisaged by end-2008. Regarding other trade arrangements, negotiations are ongoing on a customs union in the Common Market for Eastern and Southern Africa (COMESA); and discussions have begun on an Economic Partnership Agreement (EPA) with the European Union, following the finalization of an impact study.

\footnotetext{
${ }^{8}$ The last contact was in October 2006.

${ }^{9}$ The Customs Union provides for the removal of duties and nontariff barriers among members and establishes a three-band structure for its common external tariff of 0,10 , and 25 percent.
} 


\section{B. Structural Policies}

26. The focus on the structural side will remain on enhancing the productivity of the agricultural and export sectors and improving the conditions for the private sector.

\section{Our export promotion strategy will continue to focus on enhancing the} productivity in the traditional sectors and diversifying our export base.

- $\quad$ Coffee. Extension services are now available in most districts. To move into higher value added markets, the number of washing stations will be further increased while existing stations are being made more efficient.

- Tea. To increase export volumes of the remaining state-owned farms, efforts aim at reducing the cost of fertilizer, strengthening tea growing cooperatives, planting of high yielding clones, and increasing acreage under cultivation. Meanwhile, discussions are underway to accelerate privatization.

- Tourism. Private operators are expected to open eight hotels and work focuses on offering a one-week trip to extend the average stay of tourists. Moreover, a training needs assessments for the hospitality industry will be undertaken by early 2007 .

- $\quad$ Export processing Zone (EPZ). The first phase of construction, which includes the development of infrastructure, is expected to start in 2007. With respect to financing, negotiations for a grant from the AfDB to the tune of US\$22 million are ongoing.

\section{Financial sector}

28. The agenda for medium-term financial sector reform, which will be coordinated with donors in a meeting in early 2007 , focuses on:

- $\quad$ Strengthening NBR's supervisory powers. In its supervision activities, the NBR has been putting more emphasis on new banking products such as leasing (including through on-the-spot inspections). We will submit to Cabinet by end-2006 a draft law on Anti Money Laundering/Combating the Financing of Terrorism.

- Preventing a recurrence of the recent crisis in the MFI sector. We are in the process of drafting the micro finance law and will be submitting it to parliament by June 2007 (benchmark). The NBR will raise the minimum capital requirements significantly for MFIs that take deposits other than for collateral only and increase exposure limits for group loans. It will grant licenses only to MFIs with adequate management.

- $\quad$ Addressing the weaknesses of the payments system. The NBR will create by March 2007 a unit that coordinates all elements related to payments system. We will 
also by end-June 2007 reorganize and financially restructure SIMTEL with World Bank technical assistance.

- Developing long-term savings and insurances. To this end, we will draft an action plan to define the legal framework for the regulation and supervision of insurances and the contractual savings industries (including the Caisse Social du Rwanda); and the introduction of government bonds.

- Improving accounting standards. With a view to adopting IFRS (International Financial Reporting Standards), we are in the process of establishing a training program for accountants. Moreover, we will amend relevant tax laws to allow companies to base tax returns on IFRS standards.

\section{Cost of doing business}

\section{To improve the business climate and remove the obstacles to business} development as identified in the World Bank's cost of doing business index, we

- $\quad$ Are in the process of drafting 14 business related laws. While five of these laws have already been submitted to parliament, including a bankruptcy law, further laws will follow in the course of the next years, including a contract law, an e-commerce law, and a labor law.

- Will set up by March 2007 a "Commercial Justice, Business, and Land Registration Program" in partnership with the private sector, which will focus on key legal and regulatory constraints to private sector development. This will include the fair and speedy resolution of commercial disputes, including the establishment of Commercial Courts, and the provision of key business registration services, particularly for land.

- Will make operational by October 2007 the Office of the Registrar of Land Titles (benchmark).

- Will reduce the number of documents required for exports and imports by August 2007.

\section{Program Monitoring}

30. Conditionality and program reviews. The second review under the PRGF arrangement, scheduled for completion by May 30, 2007, will review quantitative performance as of end-December 2006 and structural conditionality through end-March 2007. It will focus on expenditure management and financial sector reform. The second year of the PRGF-supported program will be monitored through quantitative performance criteria for end-June and end-December 2007 and indicative targets for end-March and endSeptember 2007, as well as quarterly quantitative indicators, that will be subject to the third 
and fourth program reviews to be completed no later than end-November 2007 and end-May 2008 respectively. We have also set structural conditionality in public expenditure management, private sector development, poverty reduction, and financial sector reforms.

31. Technical memorandum of understanding (TMU). The attached TMU lays out the details of the program design and terminology. We have maintained the broad design of the program for 2006, but have added an indicative target on the net present value of external debt to ensure that our debt remains sustainable. 
Table 1. Rwanda: Quantitative Performance Criteria and Benchmarks for 2006

(Billions of Rwanda francs, unless otherwise indicated)

(Quantitative benchmarks*; and performance criteria on test dates ${ }^{\star *}$ )

\begin{tabular}{|c|c|c|c|c|}
\hline & \multicolumn{4}{|c|}{2006} \\
\hline & Mar. & Jun. ${ }^{* *}$ & Sep. ${ }^{*}$ & Dec.* \\
\hline \multicolumn{5}{|l|}{ Benchmarks and performance criteria } \\
\hline \multicolumn{5}{|l|}{ Net foreign assets of the NBR (floor on stock) ${ }^{1}$} \\
\hline Actual (program exchange rate) & 225.2 & 236.6 & 228.0 & $\ldots$ \\
\hline Adjusted program & 219.8 & 221.3 & 206.7 & $\ldots$ \\
\hline Program & 212.9 & 221.3 & 203.7 & 172.4 \\
\hline \multicolumn{5}{|l|}{ Reserve money (ceiling on stock) } \\
\hline Actual & 63.7 & 68.3 & 69.4 & $\ldots$ \\
\hline Program & 64.9 & 68.4 & 69.8 & 70.8 \\
\hline \multicolumn{5}{|l|}{ Net credit to the government (ceiling on flow) ${ }^{2}$} \\
\hline Actual & -48.9 & -47.2 & -27.7 & $\ldots$ \\
\hline Adjusted program & -30.6 & -41.5 & -27.1 & $\ldots$ \\
\hline Program & -23.8 & -41.5 & -24.1 & -10.0 \\
\hline \multicolumn{5}{|l|}{ Domestic fiscal balance (floor on flow) $)^{2}$} \\
\hline Actual & -8.3 & -29.3 & -59.2 & $\ldots$ \\
\hline Adjusted program & -19.2 & -39.1 & -60.3 & -- \\
\hline Program & -19.2 & -42.2 & -61.1 & -79.4 \\
\hline \multicolumn{5}{|l|}{ Total priority spending (floor on flow) ${ }^{2}$} \\
\hline Actual & 28.0 & 68.3 & 115.6 & $\ldots$ \\
\hline Adjusted program & 32.1 & 68.9 & 106.7 & $\ldots$ \\
\hline Program & 32.1 & 70.5 & 107.1 & 144.0 \\
\hline \multicolumn{5}{|l|}{ New nonconcessional external debt ${ }^{3}$} \\
\hline Actual & 0.0 & 0.0 & 0.0 & $\ldots$ \\
\hline Program & 0.0 & 0.0 & 0.0 & 0.0 \\
\hline \multicolumn{5}{|l|}{ New external payment arrears (ceiling on stock) ${ }^{3,4}$} \\
\hline Actual & 0.0 & 0.0 & 0.0 & $\ldots$ \\
\hline Program & 0.0 & 0.0 & 0.0 & 0.0 \\
\hline \multicolumn{5}{|l|}{ Short-term external debt (ceiling on stock) ${ }^{5}$} \\
\hline Actual & 0.0 & 0.0 & 0.0 & $\ldots$ \\
\hline Program & 0.0 & 0.0 & 0.0 & 0.0 \\
\hline \multicolumn{5}{|c|}{ Net accumulation of domestic arrears (ceiling on flow) ${ }^{2}$} \\
\hline Actual & -2.5 & -4.4 & -6.4 & $\ldots$ \\
\hline Program & -1.8 & -3.5 & -5.3 & -7.0 \\
\hline \multicolumn{5}{|l|}{ Indicative targets } \\
\hline \multicolumn{5}{|l|}{ Broad money (ceiling on stock) ${ }^{1}$} \\
\hline Actual & 221.0 & 241.2 & 258.2 & $\ldots$ \\
\hline Program & 219.5 & 224.0 & 235.7 & 243.1 \\
\hline \multicolumn{5}{|l|}{ Extended Broad money (ceiling on stock) ${ }^{1}$} \\
\hline Actual & 254.5 & 283.1 & 303.3 & \\
\hline Program & 250.8 & 256.0 & 269.3 & 277.8 \\
\hline \multicolumn{5}{|l|}{ Memorandum items: } \\
\hline \multicolumn{5}{|l|}{ General budget support (in US\$ million) ${ }^{6}$} \\
\hline Received & 28.4 & 97.7 & 122.6 & $\ldots$ \\
\hline Expected & 16.0 & 102.5 & 117.1 & 123.1 \\
\hline Of which: budget support grants (received) & 28.1 & 96.8 & 115.7 & \\
\hline Of which: budget support grants (expected) & 16.0 & 102.5 & 117.1 & 123.1 \\
\hline Budgetary "baseline grants" (TMU, para. 9$)^{6}$ & 16.0 & 95.5 & 103.1 & 103.1 \\
\hline
\end{tabular}

Sources: Rwandese authorities and IMF staff estimates and projections.

${ }^{1}$ At the program exchange rate of Rf 553.7/US\$.

${ }^{2}$ Numbers are cumulative from December 31, 2005.

${ }^{3}$ This is a continuous performance criterion.

${ }^{4}$ Excludes arrears on obligations that are subject to rescheduling

${ }^{5}$ Ceiling on outstanding stock of external debt (excluding normal imported-related credits) owed or guaranteed by the central government, local government, or the NBR with original maturity or up to, and including one year.

${ }^{6}$ Excluding external donor financing for demobilization and peacekeeping.

\section{CInternational Monetary Fund. Not for Redistribution}


Table 2. Rwanda: Quantitative Performance Criteria and Benchmarks for 2007 (Billions of Rwanda francs, unless otherwise indicated)

(Quantitative benchmarks*; and performance criteria on test dates ${ }^{\star \star}$ )

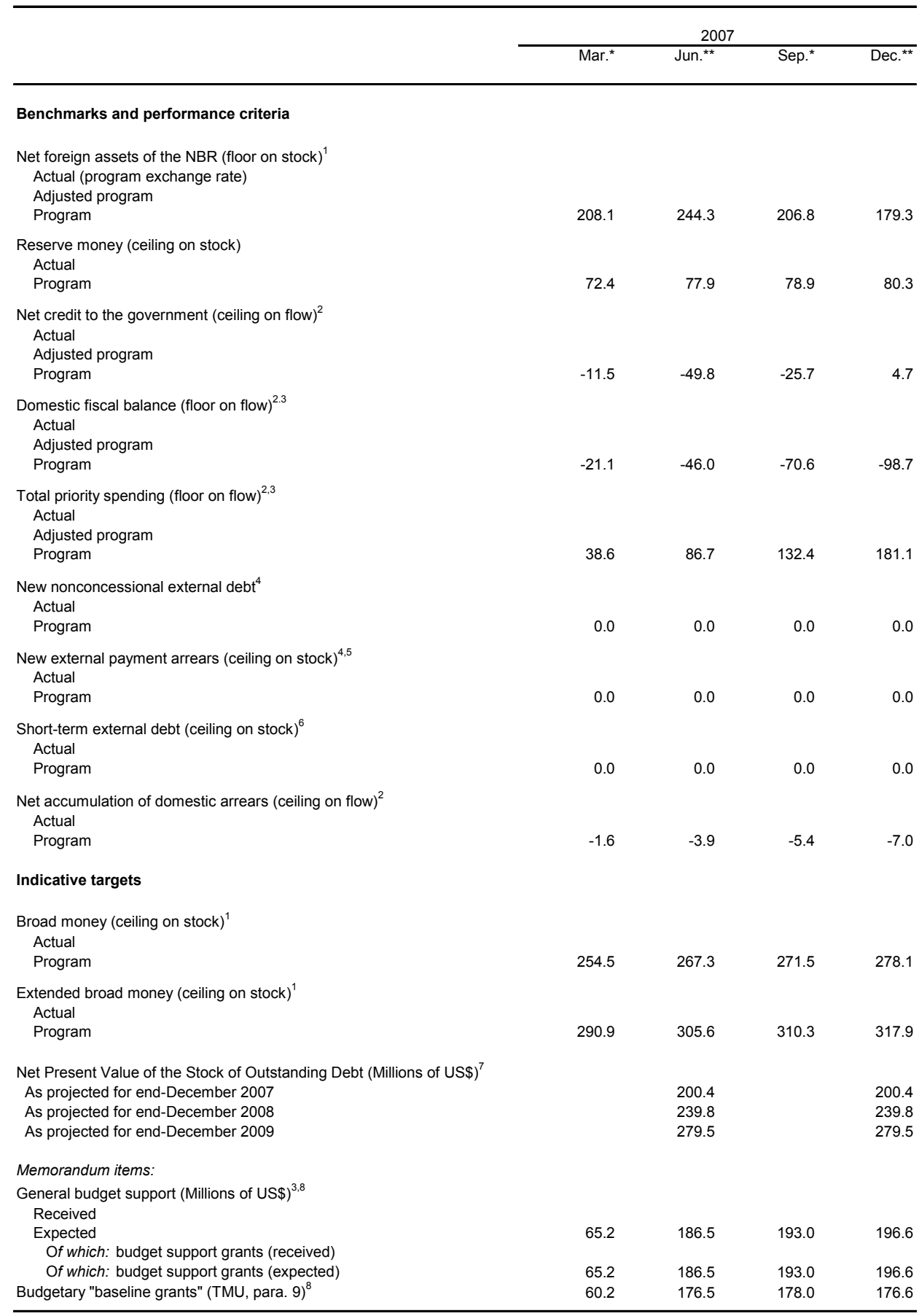

Sources: Rwandese authorities and IMF staff estimates and projections.

${ }^{1}$ At the program exchange rate of RF 549.9/US\$.

${ }^{2}$ Numbers are cumulative from December 31, 2006.

${ }^{3}$ The domestic fiscal balance and the priority spending targets will be adjusted by the amount of grants exceeding or below programmed grants.

${ }^{4}$ This is a continuous performance criterion.

${ }^{5}$ Excludes arrears on obligations that are subject to rescheduling.

${ }^{6}$ Ceiling on the stock of external debt (excluding normal imported-related credits) owed or guaranteed by the central governments, local government, or the NBR with original maturity of up to, and including one year.

${ }^{7}$ Figures indicate the NPV projections based on debt contracted at the test date.

${ }^{8}$ Excluding external donor financing for demobilization and peacekeeping.

\section{CInternational Monetary Fund. Not for Redistribution}


Table 3. Rwanda: Structural Conditionality, March-October 2007

Issuing the first report of a monitoring system of project accounts. The

Prior Action

report will show (1) the in- and outflows (the latter broken down into

foreign exchange and domestic spending) on a quarterly basis of project

accounts at the NBR listed in paragraph 46 of the TMU for July-

September 2006; and (2) rolling spending plans for the accounts on a

quarterly basis, broken down into foreign exchange and domestic

spending for January - December 2007.

\section{Public expenditure management}

Complete progress report on financial reporting by budgetary agencies

June 15, 2007

Benchmark

(line ministries, provinces, autonomous agencies). Agencies are expected to produce the following monthly financial reports: (i) bank reconciliation statement; (ii) budget execution reports, and (iii) statements of revenue and expenditure.

Publish on the Ministry of Finance's website the progress report on the OBL implementation.

Complete and publish on the Ministry of Finance's website a study on project accounts (for projects listed in paragraph 46 of the TMU) at the NBR (1) showing annual budgets with donor disbursements and spending for 2004-2006 as well as quarterly projections (including a breakdown into imports and the domestic component) for July 2007-June 2008; and (2) providing an analysis of the causes for the large accumulation of the specific project accounts since January 1, 2004.

\section{Private sector development}

Make operational the Office of the Registrar of Land Titles.

October 31, Benchmark 2007

September 30, Benchmark 2007

September 30, Performance 2007

Criterion

\section{Poverty reduction}

Produce a poverty profile of provinces, districts, and communities of Rwanda using the results of the census and the household living conditions survey.

September 30, Benchmark 2007

\section{Financial sector}

Submit to Parliament a micro finance law to bring the legal framework for microfinance broadly in line with the Guiding Principles on Regulation and June 30, 2007 Benchmark Supervision of Microfinance Institutions as published by the Consultative Group to Assist the Poorest. 


\section{APPENDIX I-ATTACHMENT II \\ RWANDA: TECHNICAL MEMORANDUM OF UNDERSTANDING}

1. This technical memorandum sets out the definitions for quantitative targets under which Rwanda's performance will be assessed and provides specifics on areas of structural conditionality. Monitoring procedures and reporting requirements as well as other program definitions are also specified.

\section{Quantitative Program Targets}

2. Performance criteria for June 30, 2007 and December 31, 2007 as well as indicative targets for March 31, 2007, and September 30, 2007 are proposed to be established with respect to:

- $\quad$ floors on the domestic fiscal balance of the central government (DFB);

- $\quad$ ceilings on the net credit to the central government (NCG);

- $\quad$ floors on priority spending;

- ceilings on the net accumulation of domestic arrears of the central government;

- $\quad$ floors on the Net Foreign Assets (NFA) of the National Bank of Rwanda (NBR);

- $\quad$ ceilings on reserve money; and

- $\quad$ ceilings on the outstanding stock of external debt with original maturities of one year or less owed or guaranteed by the public sector.

3. Performance criteria that are applicable on a continuous basis are proposed to be established with respect to the ceilings on new external payment arrears of the public sector and new nonconcessional debt of the public sector.

4. Indicative targets are proposed to be established

- $\quad$ for end-March 2007, end-June 2007 end-September 2007, and end-December 2007, for ceilings on broad money and extended broad money;

- $\quad$ for end-June 2007, and end-December 2007, for ceilings on the contracting or guaranteeing of concessional external debt. 


\section{A. Institutional Definitions}

5. The central government comprises the treasury and line ministries.

6. The public sector comprises the central government, local governments, public enterprises (including Rwandatel, Electrogaz, Ocircafé, Ocirthé, Prime Holdings, and ONP), and the NBR.

\section{B. Targets related to the Execution of the Fiscal Program}

\section{Domestic Fiscal Balance of the Central Government (DFB)}

7. A floor applies to the DFB, which is measured cumulatively from December 31, 2006 for the end-March 2007, end-June, 2007, end-September 2007 and end-December 2007 targets.

8. Definition. The domestic fiscal balance is defined as domestic revenue (excluding grants) minus domestic spending. Domestic spending is defined as current expenditure (excluding external interest due, spending related to Rwandese troops involved in the UN peace-keeping efforts (Table 1), and spending related to the World Bank-led demobilization and reintegration program) plus domestically-financed capital expenditure on a payment order basis, plus net lending.

\section{Adjusters.}

- $\quad$ The floor on the DFB will be adjusted downward (i.e., the deficit target will be increased) by the amount of the positive difference between actual and programmed budgetary grants in the context of a review with Fund staff IF this review establishes that a further fiscal expansion would not lead to a rekindling of inflation or an undue increase in domestic debt. The increase in the deficit would be used for contingent spending. A list of contingent spending of about one percent of GDP is provided in Table 1; this contingent spending will be released equally for priority and nonpriority spending. For example, if contingent spending of Rf 2 billion is released, at least Rf 1 billion should go to priority spending (so called contingent priority spending; see also paragraph 17). Any further spending in addition to the one listed in Table 1 would be for priorities only, including spending under the Fast Track Initiative for Education. ${ }^{1}$ Budgetary grants include HIPC Initiative-related grants, ${ }^{2}$ but exclude grants related to

\footnotetext{
${ }^{1}$ In the following, the contingent priority spending plus any further unprogrammed priority spending financed by additional grants will be referred to as "additional priority spending (see paragraph 17)."

${ }^{2}$ HIPC grants exclude debt relief from IDA and the AfDB.
} 
AU peace keeping activities and for the demobilization program as well as capital grants related to the development budget. Quarterly programmed budgetary grants are also provided in Table 2 of the MEFP.

- The floor on the DFB will be adjusted upward (that is, the deficit target will be reduced) by the amount actual grants are below programmed grants with a maximum adjustment of up to US\$20 million. Spending would be reduced in equal shares for priority and nonpriority spending (see also paragraph 13 ). ${ }^{3}$

- $\quad$ The floor on the DFB will be adjusted downward (i.e., the deficit target will be increased) by the amount of expenditure deemed integral to a specific privatization operation (to be recorded under net lending) IF the difference between privatization revenue and this expenditure is positive (see also paragraph 13).

- $\quad$ The floor on the DFB will be adjusted downward (i.e., the deficit target will be increased) by the amount of expenditure for food imports in the case of a food emergency (see also paragraphs 13 and 24).

10. Reporting requirement. Data on the DFB and its adjusters will be transmitted, with detailed explanations, on a monthly basis within four weeks of the end of each month.

\section{Net Credit to the Central Government (NCG)}

11. A ceiling applies to the NCG, which is measured cumulatively from December 31, 2006 for the end-March 2007, end-June 2007, end-September 2007, and end-December 2007 targets.

12. Definition. For program monitoring purposes, the NCG will be calculated as the change from end-December 2006 of net credit from the banking system and the change of holdings of treasury bills and other government securities by the nonbank sector. Net credit from the banking system is defined as the difference between:

- $\quad$ credit to government from the banking system, including credit to central government, provinces and districts, outstanding central government debt instruments; government debt to the NBR incurred as a result of the 1995 devaluation (Rf 9 billion) and the overdraft to the prewar government (Rf 2 billion); and

\footnotetext{
${ }^{3}$ Privatization receipts are recorded under nontax revenue.
} 
- $\quad$ total government deposits with the banking system of the central government, including the main treasury account, line ministries, the fund for assistance to genocide survivors, Rwanda Revenue Authority, the electoral commission, the demobilization commission, fonds routier, and any other of the 15 autonomous agencies. Thus, this definition excludes any government deposits, over which the central government does not have any direct control (i.e., for project accounts, counterpart funds, fonds publics affectés, and privatization proceeds with the NBR). ${ }^{4}$ In particular, NCG is not affected by credit to or deposits of public enterprises and autonomous public agencies.

\section{Adjusters.}

- $\quad$ The ceiling on NCG will be adjusted upward by the amount of any negative difference between actual and "baseline grants" (defined in Table 2 of the MEFP) up to a maximum adjustor of US\$30 million, evaluated in Rwanda francs at the program exchange rate.

- $\quad$ The ceiling on the NCG will be adjusted downward by the amount of privatization revenue IF the difference between this revenue and any expenditure deemed integral to the privatization operation is positive (see also paragraph 9).

- $\quad$ The ceiling on the NCG will be adjusted upward by the amount of expenditure for food imports in case of a food emergency (see also paragraph 9 and 24).

14. Reporting requirement. Data on the NCG (showing separately treasury bills and government bonds outstanding, other government debt, and central government deposits) and its adjusters will be transmitted on a monthly basis within three weeks of the end of each month. Deposits of the government with the NBR and with the commercial banks will be separated from the deposits of the public enterprises and autonomous public agencies.

\section{Priority Expenditure}

15. A floor applies to priority spending of the central government, which is measured cumulatively from December 31, 2006 for the end-March 2007, end-June 2007, endSeptember 2007, and end-December 2007 targets.

\footnotetext{
${ }^{4}$ The target excludes any transfers from the deposits over which the government has limited control into other government deposits.
} 
16. Definition. Central government priority spending is defined as the sum of those recurrent expenditures, domestically-financed capital expenditures, and net lending that the government has identified as priority in line with the PRSP process. The definition of priority expenditures is based on the program classification of the annual budget. Table 2 provides a summary of the SIBET output ${ }^{5}$ and a list of the main programs.

\section{Adjuster.}

- $\quad$ The floor will be adjusted upward by additional priority spending (see paragraph 9, footnote 1 for definition).

- $\quad$ The floor will be adjusted downward by half of the amount actual grants are below programmed grants with a maximum adjustment of up to US\$10 million.

18. Reporting requirement. Data on priority expenditure, at the same level of detail as in Table 2, will be transmitted on a monthly basis within three weeks of the end of each month.

\section{Net Accumulation of Domestic Arrears of the Central Government}

19. A ceiling applies to the net accumulation of domestic arrears of the central government, which is measured cumulatively from December 31, 2006 for the end-March 2007, end-June 2007, end-September 2007, and end-December 2007 targets. $^{6}$

20. Definition. The net accumulation of arrears is defined as the difference between the gross accumulation of new domestic arrears (measured as the difference between payment orders and actual payments related to payment orders issued) and gross repayment of any arrears outstanding on December 31, 2006 (including repayment of float in 2006 and the repayment of older arrears).

21. Reporting requirement. Data on repayment of domestic arrears and the remaining previous-year stock of arrears will be transmitted on a monthly basis within three weeks of the end of each month.

\footnotetext{
${ }^{5}$ The computerized SIBET expenditure management system tracks priority spending at the program and subprogram levels.

${ }^{6}$ A negative target thus represents a floor on net repayment.
} 


\section{Targets for Monetary Aggregates}

\section{Net Foreign Assets of the National Bank of Rwanda (NFA)}

22. A floor applies to the NFA of the NBR for the end-March 2007, end-June 2007, endSeptember 2007, and end-December 2007 targets.

23. Definition. NFA of the NBR in Rwanda francs are defined, consistent with the definition of the Special Data Dissemination Standards (SDDS) template, as external assets readily available to, or controlled by, the NBR net of its external liabilities. Pledged or otherwise encumbered reserve assets are to be excluded; such assets include, but are not limited to, reserve assets used as collateral or guarantee for third party external liabilities. Foreign assets and foreign liabilities in U.S. dollars are converted to Rwanda francs by using the U.S. dollar/Rwanda franc program exchange rate. ${ }^{7}$ Foreign assets and liabilities in other currencies are converted to U.S. dollars by using the actual end-of-period U.S. dollar/currency exchange rate. Foreign liabilities include, inter alia, use of IMF resources (CCFF and post-conflict emergency assistance purchases and SAF/ESAF/PRGF disbursements).

24. Adjusters (see paragraph 13 for symmetric adjusters to the NCG, including definitions).

- $\quad$ The floor on NFA will be adjusted downward by the amount of any negative difference between actual and "baseline grants" up to a maximum adjustor of US\$30 million, evaluated at the program exchange rate.

- $\quad$ The floor on NFA will be adjusted downward by the amount of expenditure for food imports in the case of a food emergency (see also paragraphs 9 and 13).

25. Reporting requirement. Data on foreign assets and foreign liabilities of the NBR will be transmitted on a weekly basis within seven days of the end of each week. Data on the NBR's foreign exchange liabilities to commercial banks (held as required reserves with the NBR) and the exchange rate used for their conversion into Rwanda francs will be shown separately.

\footnotetext{
${ }^{7}$ The program exchange rate for the 2007 program is set at Rf $549.9=$ US $\$ 1$ (actual exchange rate of September 30, 2006).
} 


\section{Reserve Money}

26. A ceiling applies to the stock of reserve money for the end-March 2007, end-June 2007, end-September 2007, and end-December 2007 targets.

27. Definition. Reserve money for the monetary program is defined as currency in circulation, reserves of deposit money banks (excluding NBR borrowing from deposit money banks on the money market ${ }^{8}$ but including cash in vault held by commercial banks), and deposits of public enterprises (including Caisse Sociale du Rwanda (CSR) and other autonomous public agencies (dépôts des établissements publics assimilés à l'état), deposits of nonbank financial institutions, and deposits of the private sector (autres sommes dues à la clientèle are included in reserve money). Reserve money excludes the deposits of the Caisse d'Épargne du Rwanda (C.E.R.) with the NBR up to Rf 1 billion, the dormant accounts up to Rf 1.4 billion, and import deposits placed at the NBR (cautions à l'importation) up to a maximum amount of $\operatorname{Rf} 150$ million.

\section{Adjuster.}

- $\quad$ The ceiling on the stock of reserve money will be adjusted symmetrically for a change in the required reserve ratio of commercial banks by the amount of (new reserve ratio - program baseline reserve ratio) multiplied by the reservable deposit liabilities in commercial banks.

29. Reporting requirement. Data on reserve money will be transmitted on a weekly basis within seven days of the end of each week. This transmission will include a weekly balance sheet of the NBR which will show all items listed above in the definitions of reserve money.

\section{Broad Money}

30. A ceiling applies to the stock of broad money and extended broad money for the endMarch 2007, end-June 2007, end-September 2007, and end-December 2007 targets.

31. Definition. Broad money is defined as the sum of currency in circulation, deposits in commercial banks, and nonbank deposits in the NBR. Extended broad money is defined as broad money plus deposits in credit unions and credit cooperatives (mainly UBPR).

\footnotetext{
${ }^{8}$ Borrowing by the NBR from the commercial banks on the money market is included under the net domestic assets of the NBR (netted out from commercial bank borrowing from the NBR).
} 
32. Reporting requirement. The balance sheets of the commercial banks and other banking institutions, both for the individual institutions and for the respective sector in aggregate, and the monetary survey, will be transmitted monthly within five weeks of the end of each month. The monthly transmission will also include a monthly balance sheet for the NBR, showing all items as in NBR's weekly balance sheet.

\section{Limits on External Debt}

\section{Limit on short-term external debt of the public sector}

33. A zero ceiling applies to the outstanding stock of external debt with original maturities of one year or less owned or guaranteed by the public sector or other agencies on behalf of the central government. The ceiling is measured cumulatively from December 31, 2006 for the end-March 2007, end-June 2007, end-September 2007, and end-December 2007 targets.

34. Definition. The definition of "debt", for the purpose of the limit, is set out in point No. 9 of the Guidelines on Performance Criteria with Respect to Foreign Debt (Decision No. 12274-(00/85) of August 24, 2000) and also commitments for which value has not been received. Excluded from this performance criterion are normal import-related credits, defined as liabilities that arise from the direct extension, during the normal course of trading, of credit from a supplier to a purchaser - that is, when payment of goods and services is made at a time that differs from the time when ownership of the underlying goods or services changes. Normal import credit arrangements covered by this exclusion are self-liquidating; they contain pre-specified limits on the amounts involved and the times at which payments must be made; they do not involve the issuance of securities.

35. Valuation. The amount of debt will be evaluated at the corresponding quarterly exchange rates published in the IMF's International Financial Statistics.

36. Reporting requirement: Data on debt and guarantees will be transmitted, with detailed explanations, on a monthly basis within five weeks of the end of each month.

\section{Contracting or Guaranteeing of new nonconcessional external debt of the public sector}

37. The public sector or other agencies on behalf of the central government will not contract or guarantee new nonconcessional external debt ${ }^{1}$ (as specified in paragraphs 39 and 40 ) with original maturity of more than one year. This is a continuous performance criterion.

\footnotetext{
${ }^{1}$ As the performance criterion refers to "new" debt, rescheduling or restructuring of existing debt is excluded.
} 
38. Valuation. The amount of debt will be evaluated at the corresponding quarterly exchange rates published in the IMF's International Financial Statistics.

39. Definition. This performance criterion applies to debt as defined in point No. 9 of the Guidelines on Performance Criteria with Respect to Foreign Debt (Decision No. 12274$(00 / 85)$ of August 24,2000$)$ and also to commitments contracted or guaranteed for which value has not been received. The use of Fund resources are excluded from the criterion. Included are leases and other instruments giving rise to external liabilities, contingent or otherwise.

40. For program purposes, a debt is concessional if it includes a grant element of at least 50 percent, calculated as follows: the grant element of a debt is the difference between the net present value (NPV) of debt and its nominal value, expressed as a percentage of the nominal value of the debt (i.e., the grant element is equal to the nominal value minus NPV divided by the nominal value). The NPV of debt at the time of its disbursement is calculated by discounting the future stream of payments of debt service due on this debt. The discount rates used for this purpose are the currency-specific commercial interest reference rates (CIRRs), as published by the OECD. For debt with a maturity of at least 15 years, the tenyear average CIRR will be used to calculate the NPV of debt and, hence, its grant element. For debt with maturity of less than 15 years, the six-month average CIRR will be used. To both the 10-year and the 6-month averages, the following margins for differing repayment periods should be added: 0.75 percent for repayment periods of less than 15 years; 1 percent for 15-19 years; 1.15 percent for 20-29 years; and 1.25 percent for 30 years or more.

41. Reporting requirement. Data on all new external debt, including government guarantees, will be provided on a monthly basis within five weeks of the end of each month.

\section{Contracting or guaranteeing of concessional external debt}

42. A ceiling applies to the contracting or guaranteeing of concessional (see paragraph 40) external debt of the public sector or other agencies on behalf of the central government for the end-June 2007, and end-December 2007 targets. The ceiling on the contracted debt becomes binding through a disbursement profile over 2007-09.

43. Definition and valuation. The ceiling on the contracting or guaranteeing of new concessional external debt will be measured by the U.S. dollar nominal sum of all loan agreements that have been contracted. Disbursed debt will be converted to U.S dollars, based on prevailing WEO test date exchange rates; for loans contracted but not yet disbursed, the profile disbursement will be measured at the actual exchange rate at the test date, based on the projected drawdown consistent with the medium-term fiscal framework as discussed with IMF staff. If export growth rates average about 8 percent in the next three years, Rwanda's NPV of debt- to-export ratio will be below 125 percent over the medium term and not exceed 
90 percent in 2009. The Net Present Value of debt is calculated by discounting the future stream of payments of debt service due, based on a uniform discount rate of 5 percent.

44. Reporting requirement. Data on the NPV of the stock of outstanding debt will be provided on a semi-annual basis within five weeks of the end of each month.

\section{Limit on new external payment arrears}

45. A continuous performance criterion applies to the nonaccumulation of new external payment arrears on external debt contracted or guaranteed by the public sector. External payment arrears consist of external debt service obligations (principal and interest) that have not been paid at the time they are due, as specified in the contractual agreements, but shall exclude arrears on obligations that are subject to rescheduling.

\section{STRUCTURAL CONDITIONALITY}

46. The following project accounts will be monitored on a quarterly basis (see performance criterion on the monitoring of project accounts in paragraph 17 of the memorandum of economic and financial policies): projet developpement districts et villes; $\mathrm{CDF}$ /programme de decentralisation; Minecofin - CEDP-SME Investment Fund; PDDC/MINALOC/H094 RW; Minisante Global Alliance for Vaccination; Projet Vct Integre; Global Fund Controlling Tuberculosis; Global Fund 3 HIV/AIDs; Global Fund 3 Control Malaria; The Nonproject Grant Aid Assistance; MIGEPROFE PRPAF Fonction. Cellule; RDRP/Multi-Donor Trust Fund; Basket Fund Health Human Resources; Global Fund 5 Assuring Access; and MINALOC-CDF-Financement Suisse.

\section{OThER DATA REQUiRements FOR Program MONITORING}

47. Other data will be reported on a regular basis for surveillance purposes (see Table 3). 
Table 1. Contingent spending, 2007

(in billion of Rwandese franc)

Priority spending

Rural terraces

0.10

Common development fund

0.60

Export Free Zone

0.6

Afforestation

0.10

Road fund FER

4.00

Export promotion

0.90

Expo, DBS, business support

0.40

Block grant to local governments (LABSF)

0.15

Energy

0.50

Nonpriority spending

8.5

Construction of police head office

0.5

Income generation in prisons

0.2

MININFRA (ICT)

0.4

Energy savings devises

0.4

Construction and rehabiliation of borders

0.3

Youth, culture

0.9

Feasibility studies

2.6

Across the board cuts in goods and services by 5 percent

1.9

Across the board cuts in transfers by 5 percent

1.1

Transfer to PTA bank

0.2

Total

15.8 
Table 2. Priority spending, 2007

(In millions of Rwanda francs)

\begin{tabular}{|c|c|c|}
\hline & & 2007 \\
\hline \multirow[t]{15}{*}{1 WAGES AND SALARIES } & 04 PRIMATURE & 95.00 \\
\hline & 07 MININTER & 583.20 \\
\hline & 0701 NATIONALE POLICE & $5,372.49$ \\
\hline & 09 MINAGRI & 164.83 \\
\hline & 11 MINISTR & 73.01 \\
\hline & 14 MINEDUC & 298.42 \\
\hline & 140- DECENTRALISE EDUCATION & $21,224.83$ \\
\hline & 15 MIJESPOC & 169.75 \\
\hline & 16 MINISANTE & 450.33 \\
\hline & 160- DECENTRALISE HEALTH & $3,707.62$ \\
\hline & 18 MININFRA & 331.95 \\
\hline & 20 MIFOTRA & 222.51 \\
\hline & 22 MINITERE & 282.46 \\
\hline & 23 MINALOC & 861.11 \\
\hline & Total & $33,837.51$ \\
\hline \multirow[t]{24}{*}{2 GOODS AND SERVICES } & 04 PRIMATURE & 240.40 \\
\hline & 07 MININTER & 463.81 \\
\hline & 07- DECENTRALISE PRISONS & 30.96 \\
\hline & 0701 NATIONALE POLICE & $3,028.51$ \\
\hline & 09 MINAGRI & $1,018.22$ \\
\hline & 10 MINICOM & 410.84 \\
\hline & 11 MINISTR & 376.37 \\
\hline & 14 MINEDUC & $7,023.30$ \\
\hline & 140- CAPITATION GRANT & 0.00 \\
\hline & 140- DECENTRALISE EDUCATION & $2,584.23$ \\
\hline & 140- TEXT BOOKS & $1,754.67$ \\
\hline & 15 MIJESPOC & 213.92 \\
\hline & 16 MINISANTE & $3,569.50$ \\
\hline & 160- DECENTRALISE HEALTH & 360.00 \\
\hline & 160- CONTRACTUAL APPROACH & $4,420.75$ \\
\hline & 1636 HOPITAL ROI FAYCAL (KFH) & 350.00 \\
\hline & 1638 COMMISSION NATIONALE DE LUTTE CONTRE LE SIDA & 23.53 \\
\hline & 18 MININFRA & $2,223.72$ \\
\hline & 180- ROAD FUND & $5,000.00$ \\
\hline & 20 MIFOTRA & $1,385.49$ \\
\hline & 22 MINITERE & $1,492.04$ \\
\hline & 23 MINALOC & 630.90 \\
\hline & 230- DECENTRALISE MINALOC & $1,317.20$ \\
\hline & Total & $37,918.36$ \\
\hline \multirow[t]{20}{*}{3 TRANSFERS } & 04 PRIMATURE & 0.00 \\
\hline & 0701 NATIONALE POLICE & 73.00 \\
\hline & 090- DECENTRALISED ECONOMIC DEVELOPMENT AND EMPLOYMENT & 706.00 \\
\hline & 090- REFORESTATION & $1,000.00$ \\
\hline & 090- TERRASSING & $1,000.00$ \\
\hline & 0904 OFFICE RWANDAIS DE DEVELOPPEMENT DES RESSOURCES ANIMALES ( RARL & 994.12 \\
\hline & 0912 INSTITUT DES SCIENCES AGRONOMIQUES DU RWANDA & $1,878.08$ \\
\hline & 0913 OFFICE RWANDAIS DE DEVELOPPEMENT DE L AGRICULTURE (RADA ) & 687.74 \\
\hline & 10 MINICOM & 132.88 \\
\hline & 100- EXPORT PROMOTION & $1,000.00$ \\
\hline & 1007 OFFICE RWANDAIS DE NORMALISATION & 957.01 \\
\hline & 1009 AGENCE RWANDAISE DE PROMOTION DES INVESTISSEMENTS & 897.70 \\
\hline & 1010 CENTRE D'APPUI AUX PETITES ET MOYENNES ENTREPRISES DU RWANDA(CAF & 101.00 \\
\hline & 11 MINISTR & $1,056.40$ \\
\hline & 12 MINECOFIN - FOOD IMPORT & 0.00 \\
\hline & 120-SUBSIDES TO RWADAN PRIVATE SECTOR FEDERATION & 500.00 \\
\hline & 14 MINEDUC & $1,475.79$ \\
\hline & 140- CAPITATION GRANT & $10,573.00$ \\
\hline & 1409 AGENCE DE FINANCEMENT DES BOURSES D'ETUDIANTS & $6,839.95$ \\
\hline & 1408 CONSEIL NATIONAL POUR LES EXEMENS (CNER) & $1,291.14$ \\
\hline
\end{tabular}

(C)International Monetary Fund. Not for Redistribution 
Priority spending, 2006-07 (continued)

(In millions of Rwanda francs)

1426 ECOLE DES HAUTES ETUDES DES FIANCES ET DES BANQUES

1506 REGIE DU MUSEE NATIONALE DU RWANDA

16 MINISANTE

160- DECENTRALIZED COMMUNITY CONTRACTUAL APPROACH

276.23

160- DECENTRALIZED DISTRICT HOSPITAL PERSONNEL MOTIVATION

1609 CENTRE DE TRAITEMENT ET DE RECHERCHES DU SIDA (TRAC)

$1,078.53$

432.69

465.59

1612 LABORATOIRE NATIONALE DE REFERENCE

1630 CENTRE HOSPITALIER DE KIGALI (CHK)

236.05

1631 HOPITAL NEURO PSCHIATRIQUE DE NDERA (HNN)

809.35

1633 LABORATOIRE PHARMACEUTIQUE DU RWANDA

216.85

1635 PROGRAMME NATIONAL DE TRANSFUSION SANGUINE (PNTS)

174.00

1636 HOPITAL ROI FAYCAL (KFH)

1637 HOPITAL UNIVERSITAIRE DE BUTARE

1638 COMMISSION NATIONALE DE LUTTE CONTRE LE SIDA

220.51

$1,737.37$

658.12

297.00

18 MININFRA

$1,243.87$

180- SUBSIDIES FOR RENTING POWER

180- SUBSIDIES FOR FUEL IMPORTS

$2,700.00$

$4,600.00$

20 MIFOTRA

23.00

22 MINITERE

589.62

23 MINALOC

$1,982.94$

230- DISTRICTS TRANSFER

$8,174.49$

Total

7 DOMESTIC CAPITAL

09 MINAGRI

10 MINICOM

1007 OFFICE RWANDAIS DE NORMALISATION

$3,368.31$

$2,064.00$

467.00

419.00

1008 AGENCE RWANDAISE DE PROMOTION DES INVESTISSEMENTS

650.00

12 MINECOFIN

14 MINEDUC

$1,685.00$

$1,401.00$

15 MIJESPOC

200.00

16 MINISANTE

954.00

18 MININFRA

$9,490.00$

22 MINITERE

$8,876.00$

23 MINALOC

$5,255.00$

Total

$31,461.00$

TIG

767.04

HIMO

500.00

Total

$1,267.04$

MINECOFIN / LAKE KIVU

$3,200.00$

Total 
Table 3. Reporting Requirements for Surveillance

\begin{tabular}{|c|c|c|c|}
\hline & $\begin{array}{c}\text { Frequency } \\
\text { of } \\
\text { Data }^{6}\end{array}$ & $\begin{array}{l}\text { Frequency of } \\
\text { Reporting }^{6}\end{array}$ & $\begin{array}{l}\text { Frequency } \\
\text { of } \\
\text { publication }\end{array}$ \\
\hline Exchange Rates & $\mathrm{D}$ & W & M \\
\hline International Reserve Assets and Reserve Liabilities of the Monetary Authorities ${ }^{1}$ & W & W & M \\
\hline Reserve/Base Money & W & W & M \\
\hline Broad Money & M & M & M \\
\hline Central Bank Balance Sheet & W & W & M \\
\hline Consolidated Balance Sheet of the Banking System & M & M & M \\
\hline Interest Rates ${ }^{2}$ & M & M & M \\
\hline Consumer Price Index & M & M & M \\
\hline $\begin{array}{l}\text { Revenue, Expenditure, Balance and Composition of Financing }{ }^{3}-\text { General } \\
\text { Government }\end{array}$ & M & M & M \\
\hline $\begin{array}{l}\text { Revenue, Expenditure, Balance and Composition of Financing }{ }^{3}-\text { Central } \\
\text { Government }\end{array}$ & M & M & M \\
\hline Stocks of Central Government and Central Government-Guaranteed Debt ${ }^{5}$ & A & A & A \\
\hline External Current Account Balance & A & SA & $A$ \\
\hline Exports and Imports of Goods and Services & A & $A$ & $A$ \\
\hline GDP/GNP & $A$ & SA & $A$ \\
\hline Gross External Debt & & & \\
\hline
\end{tabular}

${ }^{1}$ Includes reserve assets pledged or otherwise encumbered as well as net derivative positions.

${ }^{2}$ Both market-based and officially-determined, including discount rates, money market rates, rates on treasury bills, notes and bonds.

${ }^{3}$ Foreign, domestic bank, and domestic nonbank financing.

${ }^{4}$ The general government consists of the central government (budgetary funds, extra budgetary funds, and social security funds) and state and local governments.

${ }^{5}$ Including currency and maturity composition.

${ }^{6}$ Daily (D); Weekly (W); Monthly (M); Quarterly (Q); Annually (A); Semi-annually (SA); Irregular (I); Not Available 


\section{APPENDIX II-RWANDA: MILlENNIUM DEVELOPMENT GOALS}

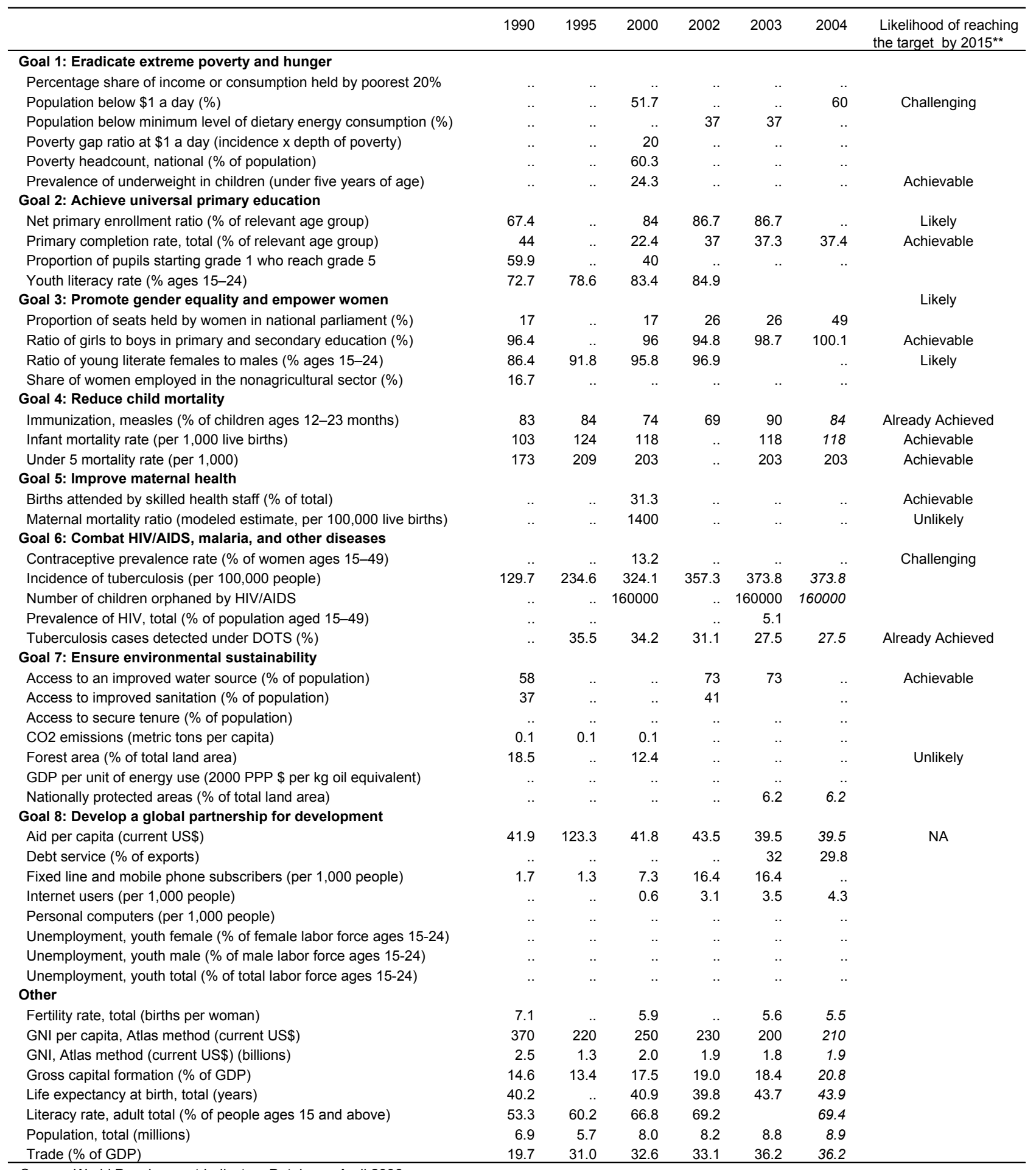

Source: World Development Indicators Database, April 2006.

Data in italics are estimates.

** World Bank Estimates. 
This page intentionally left blank

CInternational Monetary Fund. Not for Redistribution 
INTERNATIONAL MONETARY FUND

\title{
RWANDA
}

\section{Article IV Consultation, First Review Under the Three-Year Arrangement Under the Poverty Reduction and Growth Facility, and Request for Waiver of Nonobservance of Performance Criteria-Informational Annex}

\author{
Prepared by the African Department \\ (In collaboration with other departments) \\ Approved by Robert Corker and Mark Plant
}

January 10,2007

- Relations with the Fund. Describes financial and technical assistance by the IMF and provides information on the safeguards assessment and exchange system. Outstanding Fund credit was SDR2.85 million (3.56 percent of quota) at end-November 2006. Completion of the first review would allow the disbursement of SDR1.14 million.

- $\quad$ Relations with the World Bank Group. Describes World Bank Group program and portfolio and provides statement of IFC investments.

- $\quad$ Statistical Issues. Assesses the quality of statistical data. Although economic data are generally adequate for surveillance, weaknesses hamper economic analysis. 


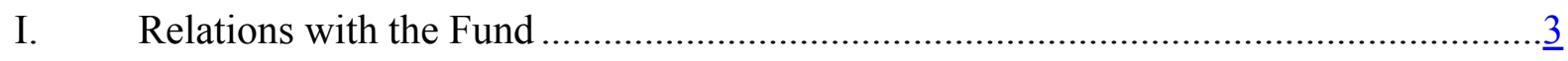

II. Relations with the World Bank Group ..............................................................

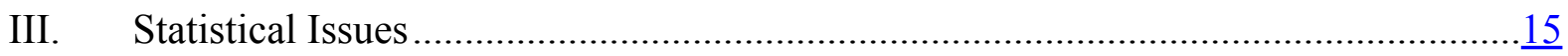

Tables:

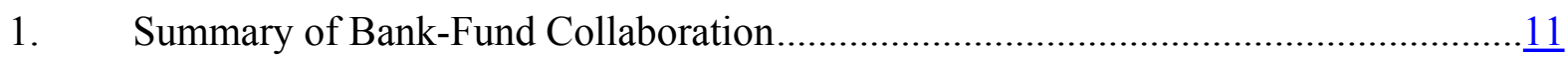

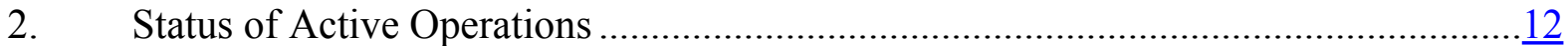

3. Table of Common Indicators Require for Surveillance............................................ 


\section{RWANDA: RELATIONS WITH THE FUND}

(As of November 30, 2006)

I. Membership Status: Joined: September 30, 1963

Article VIII

II. General Resources Account:

$\underline{\text { SDR million }}$

80.10

Quota

80.11

percent Quota

Fund holdings of currency

0.00

100.00

Reserve position in Fund

100.02

0.00

III. SDR Department:

$\underline{\text { SDR million }}$

percent Allocation

Net cumulative allocation

13.70

100.00

Holdings

15.20

110.94

IV. Outstanding Purchases and Loans:

PRGF arrangements

$\underline{\text { SDR million }}$

2.85

percent Quota

3.56

V. Latest Financial Arrangements:

$\begin{array}{lcccc}\text { Type } & \begin{array}{c}\text { Approval } \\ \text { date }\end{array} & \begin{array}{c}\text { Expiration } \\ \text { date }\end{array} & \begin{array}{c}\text { Amount } \\ \text { approved } \\ \text { (SDR million) }\end{array} & \begin{array}{c}\text { Amount drawn } \\ \text { (SDR million) }\end{array} \\ \text { PRGF } & \text { Jun 12, 2006 } & \text { Jun 11, 2009 } & 8.01 & 1.14 \\ \text { PRGF } & \text { Aug 12, 2009 } & \text { Jun 11, 2006 } & 4.00 & 4.00 \\ \text { PRGF } & \text { Jun 24, 1998 } & \text { Apr 30,2002 } & 71.40 & 61.88\end{array}$

VI. Projected Payments to the Fund (SDR million; based on existing use of resources and present holdings of SDRs):

$\begin{array}{lccccc} & \underline{5006} & \underline{2007} & \underline{2008} & \underline{2009} & \underline{2010} \\ \text { Principal } & & & & & 0.06 \\ \text { Charges/Interest } & 0.01 & 0.02 & 0.02 & 0.02 & 0.01 \\ \text { Total } & 0.01 & 0.02 & 0.02 & 0.02 & 0.07\end{array}$

VII. Implementation of HIPC Initiative:

Enhanced

framework

I Commitment of HIPC assistance

Decision point date

Dec 2000 
Assistance committed by all creditors (US\$ million) $)^{30}$

Of which: IMF assistance (US\$ million)

(SDR equivalent in millions)

46.79

Completion point date

Apr 2005

II Delivery of IMF assistance (SDR million)

Amount disbursed

Interim assistance

Completion point

Additional disbursement of interest income ${ }^{31}$

3.77

Total disbursements

50.56

\section{Implementation of MDRI Assistance}

I Total Debt Relief (SDR Million) ${ }^{32}$

Of which: MDRI

HIPIC

II Debt Relief by Facility (SDR million)

Delivery Date

January 2006
GRA

NA
PRGF Total

$52.74 \quad 52.74$

\footnotetext{
${ }^{30}$ Assistance committed under the original framework is expressed in net present value (NPV) terms at the completion point, and assistance committed under the enhanced framework is expressed in NPV terms at the decision point. Hence these two amounts can not be added.

${ }^{31}$ Under the enhanced framework, an additional disbursement is made at the completion point corresponding to interest income earned on the amount committed at the decision point but not disbursed during the interim period.

32 The Multilateral Debt Relief Initiative (MDRI) provides 100 percent debt relief to eligible member countries that are qualified for the assistance. The debt relief covers the full stock of debt owed to the Fund as of end2004 which remains outstanding at the time the member qualifies for such debt relief. The MDRI is financed by bilateral contributions and the Fund's own resources, as well as the resources already disbursed to the member under the HIPC Initiative (see Section VII above).
} 
Decision point-point at which the IMF and the World Bank determine whether a country qualifies for assistance under the HIPC Initiative and decide on the amount of assistance to be committed.

Interim assistance - amount disbursed to a country during the period between decision and completion points, up to 20 percent annually and 60 percent in total of the assistance committed at the decision point (or 25 percent and 75 percent, respectively, in exceptional circumstances).

Completion point - point at which a country receives the remaining balance of its assistance committed at the decision point, together with an additional disbursement of interest income as defined in footnote 3 above. The timing of the completion point is linked to the implementation of pre-agreed key structural reforms (i.e., floating completion point).

\section{Safeguards Assessments:}

Under the Fund's safeguards assessment policy, the National Bank of Rwanda is subject to an update of the 2003 assessment. The previous assessment concluded that substantial risks existed in the bank's safeguards framework, and recommendations made to address the main vulnerabilities have since been implemented.

\section{Exchange System:}

The Rwanda franc was pegged to the SDR until March 6, 1995, when Rwanda adopted a market-determined exchange rate system. The exchange rate has in fact behaved like a conventional fixed peg for more than a year. On December 1998, Rwanda accepted the obligations under Article VIII, Sections 2, 3 and 4 of the IMF, and maintains a system free of restrictions on the making of payments and transfers for current international transactions. In 2001, a foreign exchange auction system was put in place with technical assistance from MFD. Since February 7, 2001, auctions have been taking place on a weekly basis. The exchange rate regime is currently classified as a managed float, and the foreign exchange auctions impose a limit of $+/$ - RF 5 to the margin by which the exchange rate can vary from the previous day.

\section{Article IV Consultation:}

Rwanda is on the revised 24-month consultation cycle. The Executive Board discussed the staff report for the 2004 Article IV consultation (Country Report No. 04/382) on October 6, 2004. 


\section{FSAP Participation, ROSCs, and OFC Assessments:}

A Report on Observance of Standards and Codes on Fiscal Transparency (ROSC) was issued in July 2003. A Financial Sector Assessment Program (FSAP) has taken place in February 2005. Rwanda has not had an Offshore Financial Center (OFC) assessment.

\section{Technical Assistance:}

1999 FAD long-term experts, on tax policy, on budget preparation, and on treasury management.

1999 MFD long-term general advisor to governor of NBR.

1999 MFD experts on banking supervision and foreign exchange market operations.

2000 FAD experts on budget execution and on tax policy.

2000 MFD experts on foreign exchange market operations, and banking supervision.

2000 STA mission on money and banking statistics.

2000 STA mission on balance of payments statistics.

2001 FAD experts on expenditure management and on tax policy.

2001 FAD mission on tax policy.

2001 MFD mission on foreign exchange policy, monetary policy, and banking supervision.

2001 MFD expert on monetary policy implementation.

2001 FAD mission on assessment of tracking of poverty reducing expenditure, and the fiscal ROSC.

2001 MFD expert on banking supervision.

2001 FAD experts on expenditure management, and on tax policy (until mid-year).

2002 MFD expert on banking supervision (until November).

2002 MFD expert on monetary and foreign exchange rate policy.

2002 AFRITAC East work plan mission.

2003 AFRITAC East mission on statistical issues.

2003 AFRITAC East expert on Organic Budget Law.

2003 FAD mission on fiscal ROSC and budget management system.

2003 AFRITAC East mission on developing the market for government treasury bills.

2003 FAD mission on reform of investment incentives and tax reform.

2003 MFD expert on monetary and foreign exchange rate policy.

2003 MFD expert on banking supervision and regulation.

2003 MFD missions on banking supervision.

2003 MFD mission on foreign reserves management.

2003 STA multi-sector statistics mission.

2003 FAD mission on Decentralization.

2003 FAD mission on revenue administration.

2004 AFRITAC East expert on financial regulations.

2004 AFRITAC East mission on treasury reforms.

2004 AFRITAC East expert on implementing cash flow planning and banking arrangements.

2004 FAD tax administration expert on strengthening of revenue administration.

2004 FAD mission on revenue administration. 
2004 MFD/LEG mission on Anti-Money Laundering and Combating the Financing of Terrorism.

2004 MFD expert on banking supervision and regulation.

2004 MFD expert on monetary policy, monetary operations, and money markets.

2004 MFD missions on on-site banking supervision.

2005 AFRITAC East expert follow up on cash management.

2005 MFD/LEG mission on Anti-Money Laundering and Combating the Financing of Terrorism.

2005 MFD mission on financial statements of specific bank.

2005 MFD-WB joint FSAP mission.

2005 LEG mission on customs legislation.

2005 STA mission on Balance of Payments statistics.

2005 LEG mission on tax legislation.

2005 FAD mission on public accounting: decentralized accounting for central government.

2005 FAD expert on tax administration.

2005 MFD resident expert on monetary operations, monetary policy, money markets.

2005 MFD expert on banking supervision.

2005 LEG mission on drafting an amendment to the banking law.

2005 MFD mission on banking supervision and bank restructuring.

2006 AFRITAC East experts on establishing intergovernmental fiscal unit in the MOF.

2006 FAD advisor trade facilitation.

2006 MFD FSAP-follow up advisory mission on monetary and foreign exchange operations and NBR internal audit.

2006 LEG mission to strengthen legal and regulatory framework for bank supervision.

\section{Resident Representative:}

Mr. Lars Holger Engström assumed his duties as Resident Representative in February 2005. 


\title{
II. RWANDA: RELATIONS WITH THE WORLD BANK GROUP
}

\author{
(As of November 2006)
}

\section{Partnership for Rwanda's Development Strategy}

Donor agencies have been key players in Rwanda since the genocide. With the support from the international community, Rwanda has made notable progress along an ambitious path of reconstruction, national reconciliation, and economic reform. In recent years, the Government has made some ambitious efforts, based on its Poverty Reduction Strategy Paper (PRSP), to reduce poverty and improve living conditions of the poor. The PRSP was completed in June 2002. This strategy was supported and discussed by the Boards of the IDA and the IMF on August 12, 2002. The PRSP targeted the halving of poverty by 2015 through a private sector and rural sector strategy. The strategy mainly focuses on six priority areas: (1) rural development and agricultural transformation; (2) human development; (3) economic infrastructure; (4) good governance; (5) private sector development; and (6) institutional capacity building - as the focus for public actions on poverty reduction. Civil society, government agencies and ministries, and donors have all been actively involved in the PRSP process and monitoring. The first PRSP progress report was issued in July 2003 and a BankFund JSA produced in May 2004. The second PRSP progress report was issued in December 2004 and a Bank-Fund JSA was produced in March 2005. The third progress report of the PRSP was issued in July 2005, and the corresponding Bank-Fund JSAN was produced in March 2006.

\section{World Bank Group Program and Portfolio}

The last Country Assistance Strategy for Rwanda was discussed by the World Bank Board in December 2002. The current CAS, in support of the first PRSP, will soon come to an end, and has been extended through the preparation of an Interim Strategy Note (ISN) which was approved by the Board in September 2007. The ISN ensures that the next CAS, to be prepared jointly with DFID, is aligned with the second PRSP. Both the CAS and ISN set out an assistance program consistent with the country's PRSP and emphasize the need to move progressively from project-based approaches to budget support. In line with this approach, a Poverty Reduction Strategy Credit (PRSC) went to the Board in October 2004. This credit would help strengthen GoR capacity to (i) plan and budget results-oriented public sector actions supporting the implementation of the Poverty Reduction Strategy; (ii) develop incentive frameworks through performance-based payments and contracting; (iii) establish strong accountability mechanisms enhancing the capacity of Rwandan citizens to monitor and provide feedback to service providers - both public and private; and (iv) implement a sound fiduciary framework, as well as a monitoring and evaluation system to facilitate transparency and accountability in service delivery for the sectors of focus (i.e., health, education, water, energy). A second programmatic operation, Poverty Reduction Support Grant (PRSG), went to the Board in October 2005 and focused on (i) creating a favorable 
private sector investment climate that would promote sustained economic growth; (ii) improving quality, coverage, and equity of basic service delivery; and (iii) improving public expenditure management and governance. A third PRSG will continue with the same areas of focus and is expected to go to the Board in December 2006.

International Development Agency (IDA) Program: Since 1970, Rwanda has received 84 IDA credits and grants totaling US $\$ 1,458.6$ million. As of mid-November 2006, the active portfolio comprised twelve operations with a total net commitment value of US\$300 million, of which \$124 million remains undisbursed.

Overall, IDA has financed projects in (i) infrastructure, particularly road construction and maintenance, electricity and water supply, and sanitation infrastructure; (ii) agriculture, rural development, and forestry; (iii) social infrastructure, including health and population, and education and training; (iv) private sector development, public enterprise reform, financial development, and technical assistance; and (v) two policy-based quick-disbursing operations (IRC and PRSC1). During the immediate post-genocide period, IDA financed two emergency budget support operations and a social fund-type project, and restructured its prewar portfolio of investment projects to meet the high-priority needs associated with the emergency and the transition from conflict to development.

International Finance Corporation (IFC) Program: The IFC has made some investments in Rwanda, in various industries. Investments have been made in the Rwandan match factory (SORWAL). In FY1998, IFC approved a US\$0.53 million investment in Highland Flowers and in FY2000, a US\$6.0 million in RWANDACELL. In December 2000, an investment of US $\$ 0.8$ million was approved for an apartment hotel in Kigali. As of today, these investments with the exception of SORWAL have been cancelled. The IFC has provided some technical assistance support to Rwanda focusing on privatization, SMEs, the financial and industrial sectors. Section B below "Statement of IFC Investments in Rwanda" outlines new investments being considered by IFC.

Multilateral Investment Guarantee Agency (MIGA) Program: Rwanda signed and ratified the MIGA Convention on October 27, 1989. On September 27, 2002, it became a full member of MIGA with the completion of its membership requirements, including payment of the usable currency and the local currency portions of its initial subscription, and deposit of the promissory note. The membership was followed by Rwanda's election to MIGA's Board of Directors during the World Bank/IMF annual meetings held in Washington.

Rwanda is one of the 16 post-conflict countries in the region on which MIGA is focusing as part of its post-conflict strategy. To date, there have been no requests for guarantees coverage of projects in the country, and thus the Agency's efforts have focused on complementing the Bank Group's strategy of accelerating private sector-led growth in the country.

In consultation with the Bank, MIGA's technical assistance team undertook an assessment to assist Rwanda in creating a national investment and trade promotion capability and made 
recommendations on the proposed Rwanda Investment Authority, as well as on the medium to long-term prospects for the country to attract foreign investment. Previously, MIGA collaborated with the World Bank to advise on the establishment of the Rwanda Investment and Export Promotion Agency (RIEPA) and provided considerable information on investment prospects in the country.

In addition, MIGA's on-line investment promotion services (www.fdixchange.com and www.ipanet.net) feature approximately 90 documents on investment opportunities and the related business, legal and regulatory environment in Rwanda.

\section{World Bank staff}

Questions may be referred to Pedro Alba (Tel. 202-458-2246) and Kene Ezemenari (Tel. 202-458-5559). 
Table 1. Summary of Bank-Fund Collaboration

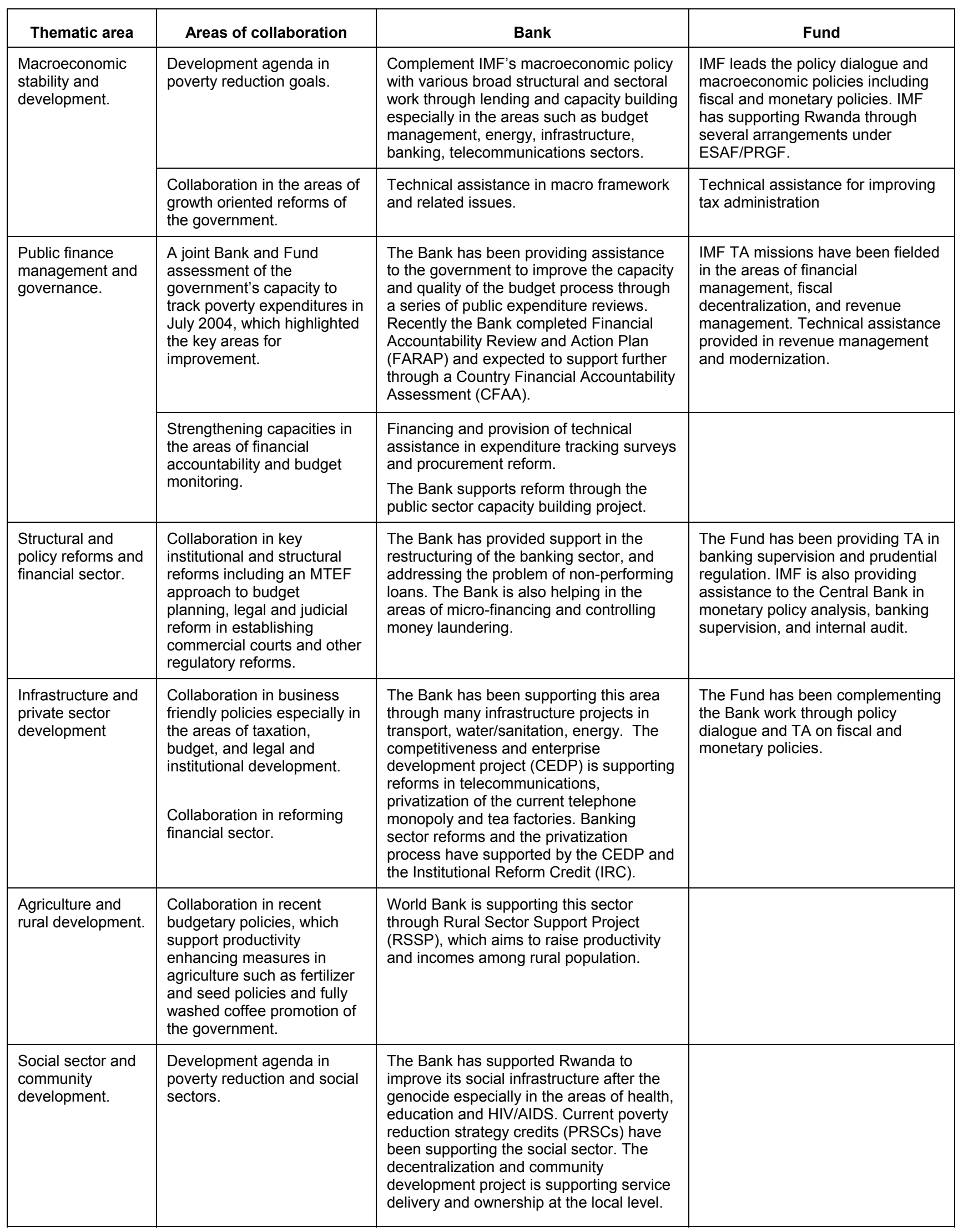


Table 2. Status of Active Operations

\begin{tabular}{|c|c|c|c|c|c|c|}
\hline \multirow[t]{2}{*}{ Project } & \multirow[t]{2}{*}{ US\$ mil. } & \multirow[t]{2}{*}{ Objective } & \multirow{2}{*}{$\begin{array}{c}\text { Approval/ } \\
\text { Effectiveness }\end{array}$} & \multirow{2}{*}{$\begin{array}{l}\text { Disbursed } \\
\text { as of } \\
\text { Nov '06 }\end{array}$} & \multicolumn{2}{|c|}{ Ratings } \\
\hline & & & & & DO & IP \\
\hline $\begin{array}{l}\text { Rural Water Supply } \\
\text { and Sanitation }\end{array}$ & 21.67 & $\begin{array}{l}\text { To increasing the availability and } \\
\text { sustainability of water supply and } \\
\text { sanitation (WSS) services in rural } \\
\text { areas; strengthening the capacity of } \\
\text { communities and agencies, and } \\
\text { mobilize community support. }\end{array}$ & $\begin{array}{l}\text { June 2000/ } \\
\text { January } 2001\end{array}$ & 14.73 & $S$ & $S$ \\
\hline $\begin{array}{l}\text { Human Resources } \\
\text { Development }\end{array}$ & 37.14 & $\begin{array}{l}\text { To build up Rwanda's human } \\
\text { resources and institutional capacity } \\
\text { to deliver education services. }\end{array}$ & $\begin{array}{l}\text { June 2000/ } \\
\text { January } 2001\end{array}$ & 25.73 & $S$ & $S$ \\
\hline Rural Sector Support & 55.87 & $\begin{array}{l}\text { To build capacity in the management } \\
\text { of farmed marshland and hillside } \\
\text { areas; access to credit and } \\
\text { competitiveness in agricultural } \\
\text { export; and small-scale rural } \\
\text { infrastructure. }\end{array}$ & $\begin{array}{l}\text { March 2001/ } \\
\text { October 2001 }\end{array}$ & 33.16 & $S$ & $S$ \\
\hline $\begin{array}{l}\text { Regional Trade } \\
\text { Facilitation }\end{array}$ & 7.93 & $\begin{array}{l}\text { To improve access to financing for } \\
\text { productive transactions and cross- } \\
\text { border trade. }\end{array}$ & $\begin{array}{l}\text { April 2001/ } \\
\text { April 2002 }\end{array}$ & 1.98 & MS & MS \\
\hline $\begin{array}{l}\text { Competitiveness and } \\
\text { Enterprise } \\
\text { Development Project }\end{array}$ & 46.15 & $\begin{array}{l}\text { To establish an enabling environment } \\
\text { for private sector-led economic } \\
\text { growth and poverty reduction in } \\
\text { Rwanda. }\end{array}$ & $\begin{array}{c}\text { April 2001/ } \\
\text { December 2001 }\end{array}$ & 35.24 & $\mathrm{~s}$ & $\mathrm{MS}$ \\
\hline $\begin{array}{l}\text { Rwanda } \\
\text { Demobilization and } \\
\text { Reintegration Project }\end{array}$ & 43.13 & $\begin{array}{l}\text { To help consolidate peace in the } \\
\text { Great Lakes region and foster } \\
\text { reconciliation within Rwanda. }\end{array}$ & $\begin{array}{c}\text { April 2002/ } \\
\text { September } 2002\end{array}$ & 25.56 & MS & $S$ \\
\hline $\begin{array}{l}\text { Multi-Sectoral } \\
\text { HIVIAIDS }\end{array}$ & 32.46 & $\begin{array}{l}\text { To strengthen prevention measures } \\
\text { in order to slow down the spread of } \\
\text { HIVIAIDS; and expand support and } \\
\text { care for those infected or affected by } \\
\text { HIVIAIDS. }\end{array}$ & $\begin{array}{l}\text { March 2003/ } \\
\text { August 2003 }\end{array}$ & 28.04 & $S$ & $\mathrm{~S}$ \\
\hline $\begin{array}{l}\text { Decentralization and } \\
\text { Community } \\
\text { Development }\end{array}$ & 20.53 & $\begin{array}{l}\text { To boost the emergence of a } \\
\text { dynamic local economy, through } \\
\text { communities who are empowered to } \\
\text { lead their own development process } \\
\text { under an effective local government. }\end{array}$ & $\begin{array}{c}\text { June 2004/ } \\
\text { December } 2004\end{array}$ & 3.92 & $S$ & $S$ \\
\hline $\begin{array}{l}\text { Public Sector } \\
\text { Capacity Building }\end{array}$ & 19.67 & $\begin{array}{l}\text { To ensure that public sector entities } \\
\text { have the capacity for more efficient, } \\
\text { effective, transparent and } \\
\text { accountable performance in their } \\
\text { redefined roles and functions and for } \\
\text { achievement of their strategic } \\
\text { objectives contributing to the } \\
\text { implementation of the PRSP }\end{array}$ & July 2004/ & 2.45 & $\mathrm{~S}$ & MS \\
\hline $\begin{array}{l}\text { Urgent Electricity } \\
\text { Rehabilitation }\end{array}$ & 24.13 & $\begin{array}{l}\text { To alleviate power shortages, and } \\
\text { enhance the capabilities of energy } \\
\text { sector institutions. }\end{array}$ & January 2005/ & 2.0 & MS & MS \\
\hline $\begin{array}{l}\text { Urban Infrastructure } \\
\text { and City Management }\end{array}$ & 21.61 & $\begin{array}{l}\text { To increase access to priority urban } \\
\text { infrastructure in Kigali and two } \\
\text { secondary cities (Ruhengeri and } \\
\text { Butare) }\end{array}$ & $\begin{array}{l}\text { November 2005/ } \\
\text { March } 2006\end{array}$ & 3.39 & $S$ & $\mathrm{~S}$ \\
\hline $\begin{array}{l}\text { East Africa Trade and } \\
\text { Transport Facilitation }\end{array}$ & 199.53 & $\begin{array}{l}\text { To improve trade environment } \\
\text { through the effective elimination of } \\
\text { tariff barriers in the EAC Customs } \\
\text { Union area; enhance logistics } \\
\text { services efficiency along key } \\
\text { corridors by reducing non tariff } \\
\text { barriers and uncertainty of transit } \\
\text { time; and improve railway services in } \\
\text { Kenya and Uganda. }\end{array}$ & January 2006/ & 8.20 & & \\
\hline E-Rwanda & 10.00 & $\begin{array}{l}\text { To improve (i) efficiency and } \\
\text { effectiveness of some internal } \\
\text { processes of the Government of } \\
\text { Rwanda, and (ii) the delivery of } \\
\text { services in selected key sectors, } \\
\text { including better access to information } \\
\text { through the use of technology. }\end{array}$ & September 2006/ & 0 & $\mathrm{HS}$ & \\
\hline
\end{tabular}

(C)International Monetary Fund. Not for Redistribution 


\section{Statement of IFC Investments in Rwanda}

With the aim of creating further investment opportunities in the Great Lakes Region and to allow the countries to benefit from economies of scale, the IFC is taking a regional approach while at the same time exploring investment opportunities. Operations under consideration are the following:

Infrastructure-Energy: IFC is exploring the possibility of participating in the financing of Kibuye Power Ltd (a Lake Kivu Methane Gas Project) under concession to Dane Associates with Wartsila as a technical partner. Project cost is estimated at US\$72 million and IFC may invest up toUS\$18 million in the project.

Infrastructure-Logistics: M/s Intraspeed SA Rwanda Ltd (ISARL) is a major freight and forwarding company operating in the Great Lakes Region of Eastern Africa. The company is involved in bulk haulage of containerized general cargo and liquid cargo from the major ports of Mombasa, Darsalaam, Tanga, and Mtwara to Kenya, Mozambique and the land locked destination countries of Uganda, Rwanda, DR Congo, Tanzania, Burundi, Zambia and Malawi. IFC approved an investment of up to US\$7.5 million on November 10, 2006 to assist the upgrade and expansion of ISARL's freight hauling capabilities.

Financial Sector: Rwanda completed the privatization of its financial sector, with the sale of the controlling shares in the Banque Commerciale du Rwanda (BCR) to the UK-based Actis Group and the Banque Continental African (BACAR) to Kenya-based FINA Bank. IFC is exploring opportunities for long term credit lines and trade finance facilities to these banks to improve access to MSME finance. IFC approved a trade finance line of up to US\$2 million for BCR. In the near term, there will be a strong role for IFC in the financial sector, in helping the development of the sector.

Tourism sector: Rwanda is developing a cluster of good quality hotels and may require IFC assistance for long term financing. IFC will consider post privatization financing of the new investors buying Hotel Intercontinental in Kigali and the Kivu Sun in Kibuye. IFC will also consider financing the rehabilitation and refurbishment of Hotel Milles de Collines.

In addition, IFC advisory and technical assistance role is expected to continue to center on the promotion of the private sector, including, the establishment of a sound enabling environment and the development of the financial sector. In this respect, IFC is implementing a leasing development program in Rwanda. Initial support consists of: (i) assisting with the review of the leasing code completed in October 2005; and (ii) contributing to a leasing financing feasibility study. Based on the outcome of the feasibility study, IFC PEP Africa has started a longer-term leasing development program with an office in Rwanda, comparable to the one currently underway in Tanzania. Also, the IFC has obtained a mandate to provide advisory services to the Government of Rwanda on the privatization of Rwanda Air. 
The IFC proposes to develop entrepreneurship in Rwanda. The program would address the needs of existing SMEs to expand their businesses, but also the needs of potential entrepreneurs. Working with institutions such as the private sector federation and business schools, the program proposes to: build capacity for business service providers; support vocational business skills development; and build capacity for entrepreneurship development and training in business schools and private sector institutions. The IFC would approach interested donors for joint support of these activities, and it is expected that the program would be in place during the first semester of calendar year 2007. 


\section{RWANDA: STATISTICAL ISSUES}

Although economic data are generally adequate for surveillance, weaknesses hamper economic analysis. National accounts and price statistics, government finance, and balance of payments statistics continue to suffer from significant weaknesses. Monetary statistics and data relevant for banking supervision are adequate for surveillance and program monitoring, but scope for improvements in quality and timeliness remains. Rwanda has participated in the General Data Dissemination System since October 2003.

Since the end of the civil war of 1994, Rwanda has received considerable technical assistance (TA) in rebuilding its statistical database, and there has been some progress in the compilation and dissemination of economic and financial statistics. In August 2005, the National Institute of Statistics (NIS) was established following the passage of the new Statistics Law.

\section{National Accounts and Price Statistics}

The national accounts are compiled and disseminated by NIS, based on 1968 System of National Accounts methodology. Quality is weak, reflecting inadequate human and material resources. While considerable effort was made to improve the reliability of GDP estimates using the production approach, significant weaknesses in data collection on expenditures, and income remain., complicating an adequate assessment of developments in savings and investment. The reliability of national accounts estimates is further hampered by weak external sector statistics.

Since 2003, the East AFRITAC has advised the authorities on real sector statistics issues, especially in secondary sector statistics. This assistance is focused on capacity building to enable the construction of short-term indicators such as a monthly PPI for the manufacturing sector. This is a joint project with the central bank (NBR). The results have not yet been integrated in the national accounts. A DFID project is also supporting the NIS with a component on national accounts, aiming to establish a program of economic surveys and the development of leading indicators that can serve as source data for national accounts estimates In February 2004 the EREBS group (Equilibre resources-emploi des biens et services) assisted the Ministry of Finance and Economic Planning (MINECOFIN) in developing new benchmarking GDP estimates (2001). Work has also advanced in the implementation of the 1993 SNA.

The consumer price index (CPI, 2003=100) utilizes expenditure weights derived from a 2000-1 survey of 6,450 households (local goods account for about 70 percent of expenditure and imported goods about30 percent; food and drink accounts for 37 percent of expenditures and housing and energy amount to 16 percent). Certain shortcomings remain, as the CPI aggregates infrequently purchased products in groups with all products in the respective group assigned the same weight.

Real sector data are reported regularly for publication in International Finance Statistics (IFS), although with some lags, particularly for GDP estimates. Data on employment and wages are not collected, except for the central government and for daily informal work. 


\section{Government Finance Statistics}

Detailed monthly revenues and expenditures are reported to AFR with a lag of three to four weeks. These data are compiled by the flash-reporting unit of MINECOFIN. A functional classification of government expenditure has been designed and presented since the 2003 budget. Within the economic classification, expenditures on PRSP designated as "priority areas" are clearly identifiable. The fiscal data do not consistently capture capital expenditure because capital projects (almost entirely externally financed) are mainly carried out by line ministries outside the regular budget process. Compilation of data on external budgetary assistance as well as on external debt would benefit from strengthened coordination between the finance ministry and the central bank. Efforts are underway to integrate the development budget into the normal budgetary procedures. Fiscal data often showed a discrepancy between above-the-line deficits and financing estimates. To address these issues with the Fund staff, the authorities have made adjustments for changes in the balance of non-core government accounts, for changes in cash in vault at the revenue authority, for accounting errors, and for other factors.

Selected aggregates on annual central government operations through 2004 have been reported to STA for publication in the IFS, But are subject to large discrepancies, largely due to the timing of recording of expenditures. No sub-annual data are reported to STA, and government finance statistics (GFS) have not been reported for publication in the GFS Yearbook since 1993.

\section{Monetary Statistics}

The balance sheet of the NBR and detailed data on money market transactions are transmitted to the AFR on a weekly basis with a lag of one week, while the monetary survey and the consolidated balance sheet of the country's commercial banks are transmitted on a monthly basis with a lag of about five weeks. Detailed data on interbank money market transactions are also provided upon request to mission staff. Monetary data are reported separately to STA and published in IFS. The data relating to the central bank are provided with a five-week lag while the data on commercial banks are reported with a delay of about three months. As a result of implementing TA recommendations, the NBR (1) adapted in early 2004 the reporting format for the banking sector closer to the methodology proposed in the Monetary and Financial Statistics Manual; and (2) is expanding the institutional coverage of the monetary survey to include credit and savings unions and microfinance institutions. Despite these efforts, inconsistencies remain in the banking sector data and a STA monetary and financial statistics mission is planned for 2007 to address outstanding issues.

The NBR is in the process of submitting monetary statistics through the Standardized Report Forms (SRFs). Following the provisional submissions by the NBR of SRFs covering January through December 2004, the NBR has completed the transition to report the 1SR for central bank data. It has not yet completed the process for the 2SR for the commercial banks and reporting under the existing framework has continued. 


\section{External Sector Statistics}

The quality of balance of payments is affected by weaknesses in the collection of source data (treatment of customs data and bank settlement reports, questionnaires) and insufficient staffing.

A June 2003 multisector statistics mission identified the following areas for improvement in the short run: (1) reorganizing data entry and production of external trade statistics, using ASYCUDA and Eurotrace software; (2) adapting survey forms sent to companies to the BPM5 methodology; and (3) collaborating with Central Public Investments and External Finance Bureau (CEPEX) to obtain data on international and bilateral aid. Subsequently, STA balance of payments statistics missions visited Kigali in January 2004 and June 2005.

Some improvements have been noted. In particular, the collection of data through direct surveys seems to be well in place, with a satisfactory rate of response, except for embassies. Also, with the assistance of these missions, the NBR has started compiling BOP/IIP statistics in conformity with international standards.

Nevertheless some weaknesses remain, in particular, the compilation of trade data relies extensively on estimates because of delays in the processing of customs declarations. The treatment of bank settlement reports is not effective, because the complete automation of the collection of declarations has not been achieved. And, finally, there is still no appropriate treatment of data on foreign official aid, for which no distinction is made between grants and loans.

Annual balance of payments and IIP data through 2005 have been reported to STA for publication in the IFS.

Databases on external public debt are maintained by both MINECOFIN and the NBR. A committee, composed of staffs from the Ministries of Finance and Economic Planning and Foreign Affairs and the NBR, is responsible for collecting, harmonizing, and monitoring information on external public debt. 
Table 3. Rwanda: Table of Common Indicators Required for Surveillance (As of December 13, 2006)

\begin{tabular}{|c|c|c|c|c|c|}
\hline & $\begin{array}{c}\text { Date of } \\
\text { Latest } \\
\text { Observation }\end{array}$ & $\begin{array}{l}\text { Date } \\
\text { Received }\end{array}$ & $\begin{array}{c}\text { Frequency } \\
\text { of } \\
\text { Data }^{6}\end{array}$ & $\begin{array}{l}\text { Frequency } \\
\text { of } \\
\text { Reporting }\end{array}$ & $\begin{array}{c}\text { Frequency } \\
\text { of } \\
\text { Publication }^{6}\end{array}$ \\
\hline Exchange Rates & Current & Current & D & W & M \\
\hline $\begin{array}{l}\text { International Reserve Assets and Reserve } \\
\text { Liabilities of the Monetary Authorities }^{1}\end{array}$ & Current & Current & W & W & $\mathrm{M}$ \\
\hline Reserve/Base Money & $9 / 30 / 06$ & 10/16/06 & W & W & M \\
\hline Broad Money & $6 / 28 / 06$ & $8 / 04 / 06$ & $M$ & M & M \\
\hline Central Bank Balance Sheet & $9 / 30 / 06$ & $10 / 16 / 06$ & W & W & M \\
\hline $\begin{array}{l}\text { Consolidated Balance Sheet of the Banking } \\
\text { System }\end{array}$ & $6 / 28 / 06$ & $8 / 04 / 06$ & $\mathrm{M}$ & $\mathrm{M}$ & M \\
\hline Interest Rates ${ }^{2}$ & $7 / 31 / 06$ & 8/28/06 & $M$ & $M$ & $M$ \\
\hline Consumer Price Index & $6 / 28 / 06$ & $7 / 20 / 06$ & M & M & M \\
\hline $\begin{array}{l}\text { Revenue, Expenditure, Balance and Composition } \\
\text { of Financing }^{3}-\text { General Government }^{4}\end{array}$ & NA & NA & NA & NA & NA \\
\hline $\begin{array}{l}\text { Revenue, Expenditure, Balance and Composition } \\
\text { of Financing }{ }^{3}-\text { Central Government }\end{array}$ & $12 / 31 / 05$ & $2 / 22 / 06$ & M & M & M \\
\hline $\begin{array}{l}\text { Stocks of Central Government and Central } \\
\text { Government-Guaranteed Debt }{ }^{5}\end{array}$ & $12 / 31 / 05$ & $2 / 22 / 06$ & $A$ & A & $A$ \\
\hline External Current Account Balance & 2005 & $8 / 24 / 06$ & $A$ & SA & $A$ \\
\hline Exports and Imports of Goods and Services & 2005 & $8 / 24 / 06$ & A & A & A \\
\hline GDP/GNP & 2005 & $2 / 22 / 06$ & A & SA & A \\
\hline Gross External Debt & & & & & \\
\hline
\end{tabular}

${ }^{1}$ Includes reserve assets pledged or otherwise encumbered as well as net derivative positions.

${ }^{2}$ Both market-based and officially-determined, including discount rates, money market rates, rates on treasury bills, notes

and bonds.

${ }^{3}$ Foreign, domestic bank, and domestic nonbank financing.

${ }^{4}$ The general government consists of the central government (budgetary funds, extra budgetary funds, and social security funds) and state and local governments.

${ }^{5}$ Including currency and maturity composition.

${ }^{6}$ Daily (D); Weekly (W); Monthly (M); Quarterly (Q); Annually (A); Semi-annually (SA); Irregular (I); Not Available (NA). 


\section{Statement by the IMF Staff Representative January 29, 2007}

1. The following information has become available since the issuance of the staff report. The thrust of the staff appraisal remains unchanged.

2. The prior action on issuing the first report of a monitoring system of project accounts was implemented on January 9, 2007.

3. Policy implementation appears to have remained broadly on track in the last quarter of 2006. Both the end-2006 targets on reserve money and net foreign assets were met. International reserves were higher than programmed, mostly on account of higher-thanprogrammed project disbursements. The authorities have indicated that draft accounting instructions, forms, and procedures for budget users will be issued within the next month, but the related end-December 2006 benchmark was not met. As foreshadowed in the staff report, the end-December 2006 benchmark for undertaking a comprehensive review of the wage structure of the public sector has not been met and is expected to be implemented by March 2007. 
This page intentionally left blank

CInternational Monetary Fund. Not for Redistribution 


\section{INTERNATIONAL MONETARY FUND}

Public Information Notice

EXTERNAL

RELATIONS

DEPARTMENT

Public Information Notice (PIN) No. 07/17

International Monetary Fund

FOR IMMEDIATE RELEASE

February 9, 2007

$70019^{\text {th }}$ Street, NW

Washington, D. C. 20431 USA

\section{IMF Executive Board Concludes 2006 Article IV Consultation with Rwanda}

On January 29, 2007, the Executive Board of the International Monetary Fund (IMF) concluded the Article IV consultation with Rwanda. ${ }^{1}$

\section{Background}

Rwanda has made great strides since the 1994 genocide. National rehabilitation has moved forward, including through presidential and legislative polls in 2003 , and community-based hearings of those accused of genocide. Output has rebounded driven mostly by a recovery in the agriculture sector. With the help of debt relief under the Heavily Indebted Poor Countries (HIPC) Initiative and Multilateral Debt Relief Initiative (MDRI), the external position has strengthened and macroeconomic stability has been largely achieved. Progress has also been made in structural reforms and Rwanda is advancing toward the Millennium Development Goals, with good results noticeable in education and gender issues. Nonetheless, poverty remains widespread and the economy remains vulnerable to climatic and terms of trade shocks.

The government of Rwanda's medium-term policies address the country's challenges to increase growth and make headway toward the Millennium Development Goals.

Macroeconomic stability and debt sustainability are considered prerequisites for strong growth.

Policies will also focus on further fostering private sector development, making the country more competitive, and removing obstacles to growth, which include low agricultural yields, limited and

\footnotetext{
${ }^{1}$ Under Article IV of the IMF's Articles of Agreement, the IMF holds bilateral discussions with members, usually every year. A staff team visits the country, collects economic and financial information, and discusses with officials the country's economic developments and policies. On return to headquarters, the staff prepares a report, which forms the basis for discussion by the Executive Board. At the conclusion of the discussion, the Managing Director, as Chairman of the Board, summarizes the views of Executive Directors, and this summary is transmitted to the country's authorities.
} 
expensive electricity supply, a poor transportation network, a shallow financing system, and high cost of doing business.

To support their policies, the authorities requested a three year Poverty Reduction and Growth Facility (PRGF) arrangement, which was approved on June 5, 2006. The program is designed to maintain macroeconomic stability while setting the stage for stronger growth and poverty reduction.

The PRGF-supported program remained broadly on track in 2006. Growth is expected to exceed 4 percent, reflecting buoyant activity in the manufacturing, services, and financial sectors. However, mostly owing to rising prices for food and energy products, 12-month inflation increased to 11 percent in November. Macroeconomic policies remain in line with the program, but short-term risks have evolved on the monetary side from a large increase in private sector credit. On the structural front, banking supervision is being strengthened, including through amendments in the banking law currently at parliament. With the passage of the Organic Budget Law in August 2006, expenditure management reforms also have picked up momentum. In addition, the authorities have been focusing on reforms to improve the business climate for the private sector and enhance productivity in the agricultural sector.

\section{Executive Board Assessment}

Directors commended Rwanda's sound macroeconomic management and progress on structural reforms and social reconstruction. Together with debt relief, this has led to macroeconomic stability, strong economic growth, and progress in advancing toward the Millennium Development Goals.

Nevertheless, Directors stressed that Rwanda continues to face major challenges in the period ahead. Poverty remains pervasive, per capita income growth has slowed in recent years, inflation pressures have resurfaced, and debt sustainability remains a concern despite HIPC and MDRI debt relief. Directors noted the limited impact of past growth on poverty reduction, and recommended that the reasons for this be explored in order to help prioritize and sequence future reforms.

Directors observed that, to boost growth and reduce poverty, the authorities will need to address Rwanda's severe infrastructure gap and low agricultural yields, enhance export competitiveness, improve the business climate, strengthen and deepen the financial sector, and tackle the skilled labour shortages. These measures will need to be backed by a prudent borrowing policy and continued reliance on concessional and grant financing, to ensure that fiscal and debt sustainability is maintained. In this context, Directors supported the authorities' strategy to keep the net present value of the public debt below 125 percent of exports over the long run.

While Directors welcomed scaled-up aid for Rwanda given the country's urgent spending needs, they underscored the importance of ensuring that macroeconomic stability is maintained. Close coordination of fiscal, monetary, and exchange rate policies will be crucial. In particular, Directors noted that increased aid is likely to intensify pressures for real exchange rate 
appreciation. They urged the central bank to follow through on its commitment to more nominal exchange rate flexibility by allowing a gradual appreciation through increased sales of foreign exchange. This would reduce the need for costly domestic sterilization to keep inflation down. Directors also stressed that the growth of private sector credit and inflationary developments need to be closely monitored, and the monetary stance kept tight until inflation is firmly on a downward path.

Directors welcomed the authorities' plans to broaden the tax base and increase priority spending in 2007. Going forward, efforts to mobilize revenue and control nonpriority spending should be further strengthened. In this context, Directors stressed that expenditure prioritization and control would be facilitated if future budgets are embedded in a medium-term expenditure framework.

Directors supported the structural reforms being implemented to improve public service delivery. The public expenditure management action plan is encouraging, but efforts should be stepped up to tackle weaknesses in expenditure management more systematically. Directors called on the authorities to move forward on civil service reform and urged prompt action to complete the review of the civil service wage structure. They also emphasized the need for increased training to build expertise and overcome capacity constraints in the public sector.

Directors welcomed the efforts to strengthen bank supervision and regulation, particularly in the microfinance sector, and looked forward to early progress in developing the medium-term financial sector reform agenda. Directors also supported the authorities' moves to improve the business environment and increase the openness of the economy, including through membership in the East African Community and other trade arrangements. In this regard, issues related to energy, transport and communications, and the cost of doing business in Rwanda would need to be vigorously addressed.

Public Information Notices (PINs) form part of the IMF's efforts to promote transparency of the IMF's views and analysis of economic developments and policies. With the consent of the country (or countries) concerned, PINs are issued after Executive Board discussions of Article IV consultations with member countries, of its surveillance of developments at the regional level, of post-program monitoring, and of ex post assessments of member countries with longer-term program engagements. PINs are also issued after Executive Board discussions of general policy matters, unless otherwise decided by the Executive Board in a particular case. 
Rwanda: Selected Economic and Financial Indicators, 2003-06

\begin{tabular}{|c|c|c|c|c|}
\hline & 2003 & 2004 & 2005 & 2006 \\
\hline & Act. & Act. & Act. & Proj. \\
\hline \multicolumn{5}{|l|}{ Output and prices } \\
\hline Real GDP (per capita) & -0.7 & 2.5 & 4.2 & 2.4 \\
\hline Consumer prices (period average) & 7.4 & 12.0 & 9.2 & 5.5 \\
\hline Consumer prices (end of period) & 7.7 & 10.2 & 5.6 & 5.0 \\
\hline Imports, f.o.b (in U.S. dollars) & 4.0 & 13.1 & 35.6 & 17.7 \\
\hline Export volume & -12.5 & 26.8 & 3.8 & 10.4 \\
\hline Import volume & 0.5 & 10.2 & 24.2 & 13.8 \\
\hline Terms of trade (deterioration $=-$ ) & 3.5 & 19.5 & 12.6 & -4.0 \\
\hline \multicolumn{5}{|l|}{ Government finance } \\
\hline Domestic credit $^{2}$ & 19.7 & -22.7 & -5.3 & 9.9 \\
\hline Government $^{2}$ & 10.7 & -28.9 & -16.4 & -4.0 \\
\hline Economy $^{2}$ & 9.0 & 6.2 & 11.2 & 13.8 \\
\hline Broad money (M2) & 15.2 & 12.1 & 17.2 & 14.5 \\
\hline Reserve money & 12.2 & 13.4 & 12.3 & 12.8 \\
\hline Velocity (GDP/M2; end of period) & 5.4 & $\begin{array}{l}5.6 \\
(\text { Perc }\end{array}$ & P) 5.5 & 5.2 \\
\hline \multicolumn{5}{|l|}{ National income accounts } \\
\hline National savings & -0.8 & 2.3 & 3.2 & 2.2 \\
\hline Gross investment & 18.4 & 20.5 & 22.6 & 21.3 \\
\hline $\begin{array}{l}\text { Of which: private (including public enterprises) } \\
\text { Government finance }\end{array}$ & 12.8 & 12.0 & 12.5 & 12.8 \\
\hline \multicolumn{5}{|l|}{ Overall balance (payment order) } \\
\hline After grants & -2.3 & -0.2 & 0.7 & -0.2 \\
\hline $\begin{array}{l}\text { Before grants } \\
\text { External sector }\end{array}$ & -10.3 & -12.1 & -13.4 & -13.6 \\
\hline \multicolumn{5}{|l|}{ External current account balance } \\
\hline Including official transfers & 7.8 & -3.0 & -3.1 & -8.4 \\
\hline Excluding official transfers & -19.2 & -18.2 & -19.4 & -19.1 \\
\hline External debt (end of period) ${ }^{5}$ & 93.4 & 91.9 & 70.7 & 14.9 \\
\hline \multicolumn{5}{|l|}{ Net present value of external debt } \\
\hline \multicolumn{5}{|l|}{ Scheduled debt-service ratio after HIPC Initiative debt relief } \\
\hline Gross reserves (in months of imports of goods and services) & \multicolumn{4}{|c|}{ (Millions of U.S. dollars) } \\
\hline External debt (end of period) $)^{5}$ & $1,572.4$ & $1,685.3$ & $1,510.0$ & 354.0 \\
\hline $\begin{array}{l}\text { Gross official reserves } \\
\text { Memorandum item: }\end{array}$ & 217.6 & 314.5 & 405.8 & 368.7 \\
\hline Nominal GDP (billions of Rwanda francs) & 905.3 & $1,054.3$ & $1,197.2$ & $1,316.1$ \\
\hline
\end{tabular}

Sources: Rwandese authorities; and IMF staff estimates and projections.

${ }_{1}^{1}$ Data based on program exchange rates.

${ }^{2}$ As a percent of the beginning-of-period stock of broad money.

${ }_{3}^{3}$ Revenue excluding grants minus current expenditure except interest due and exceptional expenditure minus domestically financed capital expenditure.

${ }^{4}$ Revenue excluding grants minus current expenditure (excluding external interest) minus domestically financed capital expenditure and net lending.

${ }^{5}$ After rescheduling, including arrears and new debt (the latter includes assumed project and budgetary disbursements for 2006) and based on

assumptions about expected new borrowing. 


\section{IMF Executive Board Completes First Review of Rwanda's Three-Year Arrangement Under the Poverty Reduction and Growth Facility}

The Executive Board of the International Monetary Fund (IMF) has completed the first review of Rwanda's performance under an SDR 8.01 million (about US\$12 million) Poverty Reduction and Growth Facility (PRGF) ${ }^{1}$ arrangement (see Press Release No. 06/121). Completion of the review enables a further release of SDR 1.1 million (about US\$1.7) which will bring total amount drawn under the arrangement to SDR 2.3 million (about US $\$ 3.4$ million).

In completing the review, the Board granted waivers for the non-observance of a quantitative performance criterion concerning total priority spending and a structural performance criterion concerning issuing a first report on a monitoring system of project accounts.

Following conclusion of the Executive Board's discussion of Rwanda's economic program, Mr. Murilo Portugal, Deputy Managing Director and Acting Chair, said:

"The Rwandan Authorities are to be commended for continuing to make good progress under their program supported by the Poverty Reduction and Growth Facility. Macroeconomic performance has continued to be satisfactory and structural reforms are broadly on track.

"Nevertheless, to reduce widespread poverty and make headway toward the Millennium Development Goals, decisive action is needed to remove obstacles to higher growth, which include low agricultural yields, a severe infrastructure gap, and an underdeveloped financial system. This will also involve making the most effective use of aid, addressing the risk of a resurgence of inflationary pressures, and prudently managing debt to maintain debt sustainability.

"Against this background, the authorities are appropriately focusing on addressing the macroeconomic policy challenges arising from scaled-up aid, while increasing spending for

\footnotetext{
${ }^{1}$ The PRGF is the IMF's concessional facility for low-income countries. PRGF-supported programs are based on country-owned poverty reduction strategies that are adopted in a participatory process involving civil society and development partners and articulated in the Poverty Reduction Strategy Paper (PRSP). This is intended to ensure that PRGF-supported programs are consistent with a comprehensive framework for macroeconomic, structural, and social policies to foster growth and reduce poverty. PRGF loans carry an annual interest rate of 0.5 percent and are repayable over 10 years with a 51/2-year grace period on principal payments.
} 
social and infrastructure priorities. In this regard, the 2007 budget is an important milestone in Rwanda's development because it judiciously increases allocations for priority areas, particularly investments in the water and energy sectors. To address the inflationary pressures stemming from increased spending and prevent costly sterilization through a build-up of domestic debt, the central bank is committed to allowing greater flexibility of the exchange rate by stepping up sales of foreign exchange. The central bank also plans to keep a tight monetary stance until inflation is firmly on a downward trend.

"The authorities' structural reform agenda is geared toward improving public service delivery and removing obstacles to growth, including by according priority to building capacity in both the civil service and the private sector. The public expenditure management plan is an encouraging step, but further progress will be needed in addressing weaknesses in expenditure management more systematically. Rwanda's infrastructure investments, reform of the banking system and overhaul of business-related laws are expected to stimulate private sector development. Going forward, it will also be important to advance trade integration and follow through on the design of medium-term financial sector reforms," Mr. Portugal said. 


\section{Statement by Laurean W. Rutayisire \\ Executive Director for Rwanda \\ January 29, 2007}

\section{I - Introduction}

At the outset, my Rwandese authorities would like to express their sincere appreciation to staff for the well-written set of papers and the candid exchange of views and constructive policy dialogue held during the discussion on the 2007 Article IV consultation and the first review under the three-year PRGF-supported program. My Rwandese authorities would also like to thank Executive Directors and Management for their continued support and advice received over the past years.

With my authorities' strong commitment to reforms and adequate Fund-supported programs, Rwanda has since the 1994 genocide made commendable progress in macroeconomic stability and implementing structural reforms, in addition to social rehabilitation. Based on these achievements my authorities reached the completion point under the enhanced HIPC in April 2005 and benefited from the MDRI debt cancellation in January 2006. In order to continue addressing challenges ahead in sustaining economic growth and reduce poverty, Rwanda embarked in June 2006 on a three-year arrangement under the PRGF with low access. As a result, macroeconomic performance is in line with the program and structural reforms are broadly on track. Indeed, all but two performance criteria for the first review were met. The quantitative end-June performance criteria on priority spending and the structural end-August performance criteria on improving the monitoring of projects accounts were missed due to technical difficulties beyond the authorities' control. As these difficulties have been resolved, the end-September target on priority spending was met and they also expect to meet the end-December target. Regarding the report of a monitoring system of project accounts, the end-August performance criterion was implemented in January 9, 2007 as a prior action.

In view of overall satisfactory program performance, my authorities request the Board's support for the completion of the first PRGF arrangement review and the waivers of nonobservance of two performance criteria. My Rwandese authorities are cognizant of the daunting challenges ahead and the need to preserve macroeconomic stability, accelerate structural reforms designed to boost growth and enhance competitiveness. The authorities are committed to maintaining sound macroeconomic policies and advancing on structural reforms. To this end they continue to count on the strong support of the international community in their efforts going forward. They are also appreciative of assistance received from their development partners, in order to help meet the challenges ahead. 


\section{II - Recent Economic Developments and PRGF Implementation}

Growth performance in Rwanda is beginning to assume a broader front in addition to the construction sector which has been the dominant force behind the economic growth experienced in recent years. Now buoyant activity is picking up in manufacturing, services and financial sectors. Economic growth is expected to reach 4 percent in 2006, despite the poor harvest in the agricultural sector due to bad rainfalls and the energy oil price shock.

In the fiscal area good performance has also been recorded. In particular revenue enhancement measures which included a widening of the tax net and a broadening of the tax base to fringe benefits resulted in revenue collection overperforming program targets and overall expenditure remained on track. As a result the end-June target on the domestic deficit was met by a wide margin. Moreover, the authorities further strengthened economic legislation, with a view to improve tax efficiency and are continuing to address weaknesses identified in implementing the Organic Budget Law. The authorities have indicated that draft accounting instructions, forms and procedures for budget users will be issued within next month while the review of the wage structure of the public sector is expected to be implemented by March 2007.

In the monetary area, targets on reserve money and net foreign assets set for end-2006 were met although inflation increased to 11 percent mainly due to the rise in food and energy prices.

The external current account for 2006 is expected to be better than programmed. On one hand, imports were lower than envisaged. On the other hand, the Banque Nationale du Rwanda (BNR) increased sterilization of government spending through domestic instruments. Exports receipts also increased, due to high volumes of coffee and tea exports. On the exchange rate front, the nominal exchange rate depreciated, although there was an appreciation in the real effective exchange rate terms.

With regard to structural reforms, implementation was broadly conducted as planned. In their efforts to bring the banking supervision in line with international best practices, the authorities submitted in September to Parliament, necessary amendments to the banking law. In addition, they closed 9 mismanaged Microfinance Institutions. To stabilize the situation and avoid adverse contagion, half of net deposits were reimbursed to depositors and measures were also put in place to administer the recovery of outstanding loans. To enhance public financial management, the authorities issued guidelines for undertaking bank reconciliation and accounting for government agencies. They also approved the publication of the Organic Budget Law and its supporting financial regulations as well as the creation of a Decentralization Unit in the Ministry of Finance. To strengthen debt management, the reconciliation of the debt databases maintained by the Ministry of Finance and the NBR was implemented. To improve the investment climate, the authorities approved the draft laws on 
establishing registration agency and on intellectual property and submitted the laws to Parliament.

\section{III - Economic Policies and Structural Reforms Going Forward}

My Rwandese authorities are fully aware of the need to pursue the implementation of prudent policies and accelerate structural reforms, geared to sustain growth, reduce poverty and achieve the remaining MDGs. Accordingly in 2007, macroeconomic policies will focus on improving the well-being of the population and growth-enhancing investments and reforms. My authorities expect growth to reach 6.5 percent, inflation brought down to 5 percent and international reserves maintained at least 4.3 months of imports. To this end they are determined to step up their efforts to remove obstacles to private sector and assign high priority to the new identified sources of growth including development of agricultural and tourism sectors as well as improvement in infrastructure and energy supply.

\section{Fiscal policy}

My authorities remain committed to prudent fiscal policy, with a view to well manage the domestic demand impact of fiscal policies. On the revenue front, the objective is to maintain the ratio of revenue-to-GDP at the level of 15 percent of GDP. Measures identified to further increase fiscal revenue include, among others, implementing tax on mobile and fixed telephone, raising the excise tax on cigarettes and as well as increasing the reference price for petroleum products. Moreover, on the basis of a survey on the informal sector, ways and means will be devised to widen the tax base over the medium-term. With regard to public expenditure, my authorities intend to increase the share of priority spending in the budget, while improving the quality of public services. Priority spending will increase for social sector and enhancing the productivity of the economy. Part of the increase in this priority spending will be financed through the MDRI debt relief. The authorities also intend to undertake an audit of the 2006 peace keeping expenditures and publish the audits results by end-March 2007, reduce to 2.37 percent of GDP the military spending, reallocate funds from tertiary to lower education and, improve the quality of education by hiring more teachers and providing districts and schools with more control of incentive schemes. A new structured electricity tariffs adopted earlier on is scheduled to be implemented early 2007 and the authorities stand ready to raise electricity tariffs if the need arises. Regarding the domestic deficit in 2007, it is projected to increase by about 1 percent of GDP, excluding spending on demobilization and peace keeping.

Capacity constraints explain the difficulties facing the authorities in the management of the fiscal area notably in the execution of the budget. To alleviate these constraints, there is a need for the Fund to play a crucial role by providing a well targeted technical assistance in collaboration with Rwanda's development partners. 


\section{Monetary and Exchange Rate Policy}

Monetary policy's objective pursued by my Rwandese authorities is to keep inflation under control. To this end, they will continue to use reserve money as the operational target. Monetary developments will be closely monitored and excess of liquidity will be sterilized through the sales of foreign exchange. For 2007, Rwandese authorities aim at reducing the inflation level to 5 percent, which will limit year-end reserve money growth to 13 percent and enable more room for credit to the private sector.

The exchange rate system adopted in 1995 has served the economy well. Rwanda accepted in 1998 the obligations under Article VIII, Sections2, 3, and 4 and maintains a system free of restrictions on payments and transfers for current international transactions. My authorities acknowledge that an increase in spending from higher aid flows is likely to result in further pressures for a real exchange rate appreciation. To address the issue, they will forcefully implement the NBR's intervention strategy with a view not to rekindle inflation or crowd out private investment. In their efforts to further promote the market-oriented system, my authorities have started to pave the way for an interbank-based market. In this respect, an instruction was issued last June to improve auction procedures and abolish the provision allowing the NBR to check whether the foreign exchange purchased at auction was used in accordance with regulations. Amendments to the foreign exchange regulations were also issued in order to address regulatory constraints and reduce reporting requirements. Moreover, on the issue of further selling foreign exchange in order to mop up excess of liquidity and preserve Rwanda's competitiveness, my authorities are of the view that this approach should be gradual and close coordination of fiscal, monetary and exchange rate policies should be made with a view to help address the volatility of external aid which continues to be crucial. My authorities cognizant of the need to further deepen the foreign exchange market and to this end call on the Fund to provide technical assistance designed to help assess the framework and operational guidelines of the existing market. This assessment will enable my authorities to enhance the dialogue with staff on the issue regarding the flexibility of the exchange rate.

\section{Financial sector issues}

The financial sector has improved over the recent years and authorities are determined to enhance banking supervision, including supervision of microfinance institutions through the implementation of recommendations made by the 2005 FSAP. In addition to further strengthening financial institutions, the authorities aim at increasing the savings rate and open up access to credit. In order to prevent the recurrence of crisis in the microfinance sector, a law on microfinance institutions will be submitted to Parliament by June 2007. The minimum capital requirements for these institutions will be increased, as well as exposure limits for group loans and adequate management will be required in granting licenses. Regarding the modernization of the payment system, a unit was created in March 2006 to 
coordinate the various components of the process. My authorities also intend to reorganize and financially restructure SYMTEL with the World Bank's technical assistance. As for the promotion of the insurances' industry, an action plan designing the framework for the regulation and supervision has been launched. Furthermore and in order to adopt the international financial reporting standard (IFRS), the authorities have initiated a training program for accountants and intend to amend relevant tax laws, with a view to allow companies to base tax returns on IFRS standards.

\section{External sector and debt issues}

The authorities through their export promotion strategy will continue to focus on enhancing the productivity in the traditional sectors and diversify the country's export base. On the external debt front, it is worth noting that the implementation of debt relief under the enhanced HIPC and MDRI is at an advanced stage. The indicators of Rwanda's debt sustainability are more favorable than at the last program review. Most of the creditors have provided debt relief to Rwanda or are willing to cancel outstanding loans. Taking fully into account these achievements, my Rwandese authorities remain committed to pursuing a prudent debt management policy. In this regard, a new debt recording system linking the Treasury and the NBR will be in place early 2007. An indicative target on the Net Present Value of external debt for 2007 will be introduced, in order to ensure the debt sustainability and allow a review of the medium-term indebtedness profile.

\section{Regional trade and integration}

My Rwandese authorities are cognizant of the importance of regional trade and integration in the country's development strategy. Benefits expected from the country' participation in the promotion of regional trade and economic integration include diversification of the economy through broadening export base and enhancing capacity to finance the development efforts. In this context and since November 2006 Rwanda became a member of the East African Community. The terms of the treaty and customs union will be negotiated in 2007 and implemented by end-2008. Moreover, negotiations are also ongoing on a customs union in the Common Market for Easter and Southern Africa in addition to discussions on an Economic Partnership Agreement with the European Union. It is worth noting that Rwanda's participation in all these arrangements will enable the country to promote its external trade, address common issues including infrastructure needs and free movement of goods, people and services.

\section{Structural reforms and competitiveness}

My authorities are determined to pursue their structural reform agenda, with a view to tackle obstacles identified on the path towards a strong and sustainable economic growth. Among these obstacles, they note low agricultural yields, severe infrastructure gap, a shallow financial system and high cost of doing business. Therefore, improvements in the country's 
competitiveness will hinge on pursuing structural reforms geared to boost the productivity of agricultural and export sectors as well as improving the conditions of the private sector promotion. In this respect the authorities will enhance the dialogue with all stakeholders and further strengthen the link between policies and growth and poverty alleviation. In their efforts to create conducive environment for investment and private sector promotion, my Rwandese authorities have submitted to Parliament five out of 14 business laws including a bankruptcy law. They plan to introduce in the course of the years to come laws pertained to contract enforcement, e-commerce and labor. They also plan to establish by March 2007 a program on "Commercial Justice, Business and Land Registration" in partnership with the private sector, make operational by October 2007 the Office of the Register and Land Titles and reduce by August 2007 the number of documents required for exports and imports.

In addition my authorities are mindful that factors other than exchange rate policy are important in determining the level of competitiveness of the economy. In particular my authorities are committed to improving the business environment, implementing civil service reforms, investing in infrastructure, enhancing agricultural productivity, promoting new information technologies and communication networks in order to increase the supply responsiveness of the economy.

\section{IV - Conclusion}

In order to continue making significant progress in reducing poverty and reaching the MDGs, my Rwandese authorities are fully committed to policies and reforms set out in the current PRGF-supported program. With the continued support of the Fund and the international community, commendable achievements have been made in addressing the country's difficult challenges. Nevertheless, a lot remains to be done in order to sustain growth, improve competitiveness and alleviate poverty in a context of limited resources. My authorities are hopeful that strong and continued technical and financial assistance from the international community will be forthcoming. On their behalf, I will appreciate the Board's support for the completion of the first review under the PRGF for Rwanda and waivers for nonobservance of two performance criteria. 